\title{
1. Transport of Mass, Momentum and Energy in Planetary Magnetodisc Regions
}

\author{
Nicholas Achilleos • Nicolas André • \\ Xochitl Blanco-Cano • Pontus C. Brandt . \\ Peter A. Delamere • Robert Winglee
}

Received: 3 February 2014 / Accepted: 5 August 2014 / Published online: 2 October 2014

(C) The Author(s) 2014. This article is published with open access at Springerlink.com

\begin{abstract}
The rapid rotation of the gas giant planets, Jupiter and Saturn, leads to the formation of magnetodisc regions in their magnetospheric environments. In these regions, relatively cold plasma is confined towards the equatorial regions, and the magnetic field generated by the azimuthal (ring) current adds to the planetary dipole, forming radially distended field lines near the equatorial plane. The ensuing force balance in the equatorial magnetodisc is strongly influenced by centrifugal stress and by the thermal pressure of hot ion populations, whose thermal energy is large compared to the magnitude of their centrifugal potential energy. The sources of plasma for the Jovian and Kronian magnetospheres are the respective satellites Io (a volcanic moon) and Enceladus (an icy moon). The plasma produced by these
\end{abstract}

\footnotetext{
N. Achilleos ( $)$

Department of Physics and Astronomy, Centre for Planetary Sciences, University College London, London, UK

e-mail: nicholas.achilleos@ucl.ac.uk
}

\section{N. André}

Institut de Recherche en Astrophysique et Planétologie (IRAP)/Centre National de la Recherche Scientifique (CNRS), Université de Toulouse, Toulouse, France

e-mail: nicolas.andre@irap.omp.eu

\section{Blanco-Cano}

Instituto de Geofísica, Universidad Nacional Autónoma de México, Ciudad Universitaria, Mexico City, Mexico

e-mail: xbc@geofisica.unam.mx

P.C. Brandt

Johns Hopkins University Applied Physics Laboratory, Laurel, MD, USA

e-mail: pontus.brandt@jhuapl.edu

\section{P.A. Delamere}

Geophysical Institute, University of Alaska Fairbanks, Fairbanks, AK, USA

e-mail: Peter.Delamere@gi.alaska.edu

R. Winglee

Department of Earth and Space Sciences, University of Washington, Seattle, WA, USA

e-mail: winglee@ess.washington.edu 
sources is globally transported outwards through the respective magnetosphere, and ultimately lost from the system. One of the most studied mechanisms for this transport is flux tube interchange, a plasma instability which displaces mass but does not displace magnetic flux-an important observational constraint for any transport process. Pressure anisotropy is likely to play a role in the loss of plasma from these magnetospheres. This is especially the case for the Jovian system, which can harbour strong parallel pressures at the equatorial segments of rotating, expanding flux tubes, leading to these regions becoming unstable, blowing open and releasing their plasma. Plasma mass loss is also associated with magnetic reconnection events in the magnetotail regions. In this overview, we summarise some important observational and theoretical concepts associated with the production and transport of plasma in giant planet magnetodiscs. We begin by considering aspects of force balance in these systems, and their coupling with the ionospheres of their parent planets. We then describe the role of the interaction between neutral and ionized species, and how it determines the rate at which plasma mass and momentum are added to the magnetodisc. Following this, we describe the observational properties of plasma injections, and the consequent implications for the nature of global plasma transport and magnetodisc stability. The theory of the flux tube interchange instability is reviewed, and the influences of gravity and magnetic curvature on the instability are described. The interaction between simulated interchange plasma structures and Saturn's moon Titan is discussed, and its relationship to observed periodic phenomena at Saturn is described. Finally, the observation, generation and evolution of plasma waves associated with mass loading in the magnetodisc regions is reviewed.

Keywords Magnetodiscs $\cdot$ Magnetospheres $\cdot$ Plasma $\cdot$ Ion-cyclotron waves $\cdot$ Magnetic reconnection

\section{Overview}

The rapidly rotating magnetospheres of the 'gas giant' planets, Jupiter and Saturn, combined with the relatively small angles between the magnetic and rotational axes of those worlds, are the principal features which lead to the formation of so-called 'magnetodisc' regions. As the name suggests, the magnetic field in these regions of the magnetosphere may be visualised with dipolar lines of force which have been 'stretched' radially outwards near the equatorial plane. Gledhill (1967) was among the first to show that centrifugal force would confine plasma into a 'disc-like' configuration in a system with a dipole-like, rigidly rotating field.

The most general representation of force balance in a rotating plasma takes into account plasma pressure gradient (including anisotropy), centrifugal force and magnetic (' $\mathbf{J} \times \mathbf{B}$ ') force. In this general case, we may write the following equations for balance both parallel and perpendicular to the magnetic field $\mathbf{B}$. These forms of the equations, as we shall see, more intuitively illustrate the role of the magnetic field geometry. The field-aligned equation of balance, firstly, may be written:

$$
-\frac{d P_{\|}}{d s_{\|}}+\frac{\left(P_{\|}-P_{\perp}\right)}{B} \frac{d B}{d s_{\|}}+N_{i} m_{i} r \omega^{2} \hat{\boldsymbol{\rho}} \cdot \hat{\mathbf{b}}=0 .
$$

The symbols have the following meanings: field-parallel pressure $P_{\|}$, field-perpendicular pressure $P_{\perp}$, magnetic field strength $B$, arc length along magnetic field line $s_{\|}$, ion number density $N_{i}$, ion mass $m_{i}$, cylindrical radial distance $r$ (measured perpendicular to the axis of rotation), plasma angular velocity $\omega$, unit vectors along the cylindrical radial direction, $\hat{\boldsymbol{\rho}}$, and magnetic field direction $\hat{\mathbf{b}}$. Reading from left to right, the terms 
in (1) represent the parallel pressure gradient, the mirror force (arising from pressure anisotropy) and the centrifugal volume force (we have neglected the contribution of the electron mass in this term). An isotropic, non-rotating plasma would simply be described by $-\frac{d P_{\|}}{d s_{\|}}=0$, i.e. uniform pressure all along any field line. The additional forces impose structure on the plasma pressure distribution, and the centrifugal term in particular is effective at confining cold plasma towards the rotational equator (e.g. Caudal 1986; Kivelson and Southwood 2005). Equation (1) is a fluid equation applicable to a quasi-neutral plasma. Equations which separately describe the field-parallel force balance for ions and electrons may also be derived. These would contain terms corresponding to ambipolar electric fields (see, for example, Appendix C in Achilleos et al. 2010; Maurice et al. 1997).

If we now consider force balance in a direction orthogonal to $\mathbf{B}$, we may write:

$$
\left(\frac{\left(P_{\|}-P_{\perp}\right)}{R_{c}}\right) \hat{\mathbf{R}}_{c}-\nabla_{\perp}\left(P_{\perp}\right)-\nabla_{\perp}\left(\frac{B^{2}}{2 \mu_{o}}\right)-\left(\frac{B^{2}}{\mu_{o} R_{c}}\right) \hat{\mathbf{R}}_{c}+N_{i} m_{i} r \omega^{2} \hat{\boldsymbol{\rho}}_{\perp}=0,
$$

where the additional symbols have the following meanings: field line radius of curvature $R_{c}$, unit vector along this radius of curvature $\hat{\mathbf{R}}_{c}$, component of gradient operator perpendicular to the field $\nabla_{\perp}$, magnetic vacuum permeability $\mu_{o}$, component $\hat{\boldsymbol{\rho}}_{\perp}$ of vector $\hat{\boldsymbol{\rho}}$ in the direction perpendicular to $\mathbf{B}$. Reading from left to right, the terms in (2) represent the anisotropy force, perpendicular pressure gradient, magnetic pressure gradient, magnetic curvature force and the centrifugal force component perpendicular to the field. Kivelson and Southwood (2005) pointed out the importance of the pressure anisotropy in the Jovian system, where rotating magnetic flux tubes may become significantly radially distended on the time scale required by ions to travel along them, from polar to equatorial regions. This process may cause the anisotropy term in (2) to grow and violate the force balance conditions (we will return to this aspect in more detail below). When this happens, the corresponding flux tube loses its integrity and 'blows open', thus forming an important channel of mass loss from the magnetosphere. In a broader context, this type of mass loss process, driven by rotation and pressure anisotropy, may also be operating in other astrophysical bodies, such as magnetised accretion discs or proto-stellar discs.

A useful, albeit greatly simplified, representation of force balance in the magnetodisc is obtained if we commence with (2), assume isotropic pressure $\left(P_{\perp}=P_{\|}=P\right)$ and apply it at the rotational equator of a system whose magnetic and rotational axes of symmetry are coincident. That is, where the vectors $\hat{\mathbf{R}}_{c}$ and $\hat{\boldsymbol{\rho}}$ are equal, and both perpendicular to $\mathbf{B}$. Under these simplifying assumptions, we may represent the balance between centrifugal force, plasma pressure gradient, and magnetic force along the equatorial, radial direction $(r)$ as follows:

$$
N_{i} m_{i} r \omega^{2}-\frac{d P}{d r}-\frac{d}{d r}\left(\frac{B^{2}}{2 \mu_{0}}\right)-\frac{B^{2}}{\mu_{0} R_{c}}=0 .
$$

This equation is appropriate for a simple, rigid plasmadisc which lies entirely in the equatorial plane (e.g. Caudal 1986; Kivelson and Southwood 2005). Achilleos et al. (2010) constructed such isotropic-pressure models for the Jovian and Kronian discs, and noted the following general trends for the various force terms. For average conditions in Saturn's magnetodisc, the total plasma pressure gradient exceeds the centrifugal volume force in the equatorial distance range $\sim 8-12 \mathrm{R}_{\mathrm{S}}$, outside of which centrifugal force is dominant $\left(R_{S}=60268 \mathrm{~km}\right.$ denotes Saturn's 1-bar equatorial radius). They noted, however, that the strong variability in hot plasma pressure at Saturn could easily change this picture. For the average conditions at Saturn, the total plasma beta falls below unity at distances inside $\sim 8 \mathrm{R}_{\mathrm{S}}$. The Jovian disc models (incorporating Voyager observations) in this study 
Fig. 1 Structure of Jovian magnetodisc model. Upper panel: Magnetic field lines (dark curves) lying in the magnetic meridian plane, with $\rho$ and $Z$ denoting cylindrical radial and vertical distance, respectively. The superposed grey curves are contours of the ratio $\xi$ of the energy density of magnetic field to that of the plasma rotation (see text). Lower panel: As for the upper panel, but now with contours of the decimal logarithm of the magnetic field magnitude in nano-Tesla. Spacing between adjacent contours is 0.2 units. For clarity, some labels are omitted

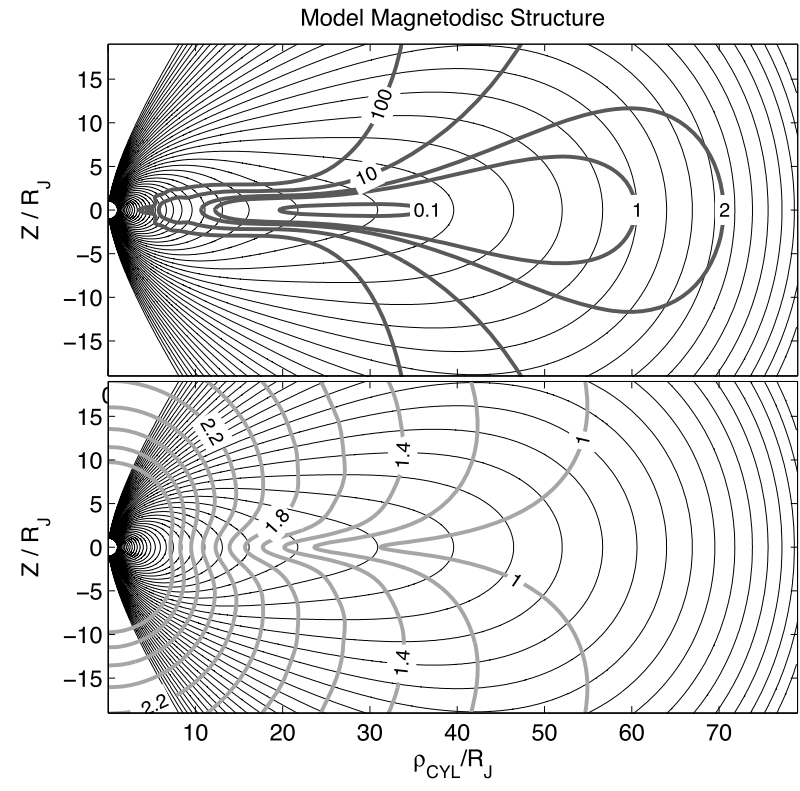

showed comparable plasma pressure gradient and centrifugal forces in the distance range $\sim 20-30 \mathrm{R}_{\mathrm{J}}\left(\mathrm{R}_{\mathrm{J}}=71492 \mathrm{~km}\right.$ denotes Jupiter's 1-bar equatorial radius), with plasma pressure gradient dominating outside this interval. Total plasma beta falls below unity at distances inside $\sim 12 \mathrm{R}_{\mathrm{J}}$.

If we wish to approximate the region where centrifugal stress alone is large enough to radially displace the planetary dipolar field lines, a simple first approach is to require that the energy density of the field be less than the kinetic energy density of plasma rotation. Using the same symbols as above, we may write:

$$
\frac{B^{2}}{\mu_{0}} \lesssim N_{i} m_{i} r^{2} \omega^{2},
$$

which is equivalent to requiring that the rotational velocity of the plasma, $V_{\phi}=r \omega$, be transAlfvénic.

In Fig. 1, we show in the top panel the magnetic field lines for a cylindrically symmetric model of Jupiter's magnetosphere, based on the force-balance formalism of Caudal (1986). Superposed on the field lines are contours of the quantity $\xi=\left(B^{2} / \mu_{0}\right) /\left(N_{i} m_{i} r^{2} \omega^{2}\right)$. The region where this ratio $\xi$ is smaller than unity encloses a disc-like body of centrifugally confined cold plasma, extending between $\sim 10-60 \mathrm{R}_{\mathrm{J}}$ (in the present context, 'cold' plasma may be defined as a medium in which the thermal energy of particle motions is small compared to their kinetic energy of bulk rotation). The lower panel, extracted from the same model, shows that the cold plasmadisc has a weaker field strength at its centre than in the neighbouring 'lobe' regions which are relatively devoid of plasma. This property reflects the balance between thermal plasma pressure inside the disc and magnetic pressure outside. Repeating this exercise for the model of Saturn's magnetodisc by Achilleos et al. (2010) reveals a similar structure, with the ratio $\xi$ having a maximum value about half that of the Jovian model, with equatorial value below unity from $\sim 8 R_{S}$ nearly out to the magnetopause.

This disc-like field structure is supported by a distributed azimuthal current or ring current. On a microscopic scale, the ring current is produced by different ion and electron drift 
motions, which are associated with the macroscopic forces summarised in (3) (e.g. Caudal 1986; Achilleos et al. 2010; Bunce et al. 2007). One of these drift motions is the inertial drift due to the centrifugal force which arises in a frame of reference that corotates with the cold plasma. The intensity of the corresponding contribution to the ring current will depend on the local angular velocity of the plasma, which is represented as the quantity $\omega$ in (3). In situ observations of the plasma angular velocity, $\omega$, were first reported for Jupiter by McNutt et al. (1981) (Voyager observations), and for Saturn by Lazarus and McNutt (1983), later augmented by Wilson et al. (2008); Kane et al. (2008). Studies by Kellett et al. (2010, 2011) are among the most detailed analyses of the morphology of Saturn's ring current and the contributions to this current associated with bulk rotation and thermal pressure of the magnetospheric plasma.

For both Jupiter and Saturn, the fact that the plasma rotation rate generally decreases with radial distance is an important observational signature of the interaction, or coupling, between the magnetospheric disc and the planet's ionosphere. Hill (1979) developed a theory for this coupling process in the Jovian system. He described the link between the intensity of field-aligned currents flowing between the ionosphere and the magnetosphere, and the radial profile of plasma angular velocity. In this picture, there are two major factors which determine the dependence of $\omega$ on radial distance. The first is related to the radial transport of angular momentum in the plasmadisc. The original source of this momentum is the plasma originating from the Io torus, which, in Hill's theory, is assumed to form a corotating (with the planet) 'inner boundary' for the disc. As the plasma diffuses radially outwards, via processes to be later described, its tendency is to conserve angular momentum, and hence for $\omega$ to decrease with increasing radial distance $r$. In the absence of other forces, the disc plasma would thus exhibit an angular velocity profile $\omega \propto r^{-2}$.

In reality, there is an additional force on the plasma, which arises from the tendency of the most radially distant (equatorial) segments of outward-moving flux tubes to be 'bent back' against the direction of plasma rotation as $\omega$ decreases. This action produces a significant, non-zero azimuthal component $B_{\phi}$ in the magnetic field ( $B_{\phi}<0$ north of the magnetodisc current sheet in the equatorial plane, and $B_{\phi}>0$ in the south). The corresponding curl of the field is equivalent to a radial current density $J_{r}$ flowing outwards through the middle magnetosphere. The magnetic force per unit volume exerted on the disc plasma is the second factor which determines the angular velocity profile, and may be written as $-J_{r} B_{z} \mathbf{e}_{\phi}$, where the $z$ direction is orthogonal to the current sheet, $B_{z}<0$ (southward-pointing) for both Jupiter and Saturn, and $\mathbf{e}_{\phi}$ is a unit vector pointing in the local direction of planetary corotation. It follows that the magnetic force acts to accelerate the plasma back towards corotation with the planet. The origin of this force is the angular momentum of the planet itself. This is because the radial current which flows in the magnetospheric current sheet is part of a larger current system which closes through the planet's ionosphere, by means of field-aligned currents that flow between these two regions (Fig. 2). The ionospheric current is driven by ion-neutral collisions. Hence, the angular momentum of the planet's rotating thermosphere is transferred to the magnetospheric plasma by a field-aligned current system.

Taking into account these two mechanisms for angular momentum transport in the Jovian magnetosphere, and assuming a pure dipolar magnetic field with an axis of symmetry aligned with the planet's rotational axis, Hill's (1979) steady-state equation for the change in plasma specific angular momentum $\ell$ with radial distance is:

$$
\begin{aligned}
\frac{d \ell}{d L} & =\omega_{J} \mathrm{R}_{\mathrm{J}}^{2} \frac{d}{d L}\left[L^{2}\left(\frac{1-\delta \omega}{\omega_{J}}\right)\right] \\
& =4 \pi\left(\frac{\Sigma}{\dot{M}}\right) \delta \omega \mathrm{R}_{\mathrm{J}}{ }^{4} \mathrm{~B}_{\mathrm{J}}{ }^{2} \frac{(1-1 / L)^{1 / 2}}{L^{3}}
\end{aligned}
$$




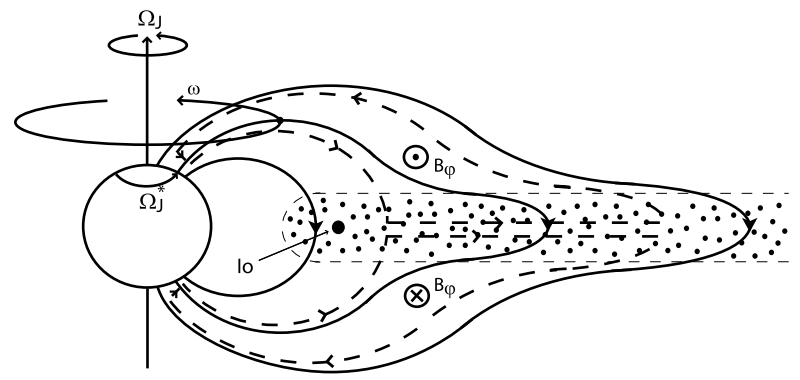

Fig. 2 Figure and caption reprinted from Cowley and Bunce (2001), Copyright (2001), with permission from Elsevier: Sketch of a meridian cross-section through the Jovian magnetosphere, showing the principal features of the inner and middle magnetosphere regions. The arrowed solid lines indicate magnetic field lines, which are distended outwards in the middle magnetosphere region by azimuthal currents in the plasma sheet. The plasma sheet plasma originates mainly at Io, which orbits in the inner magnetosphere at $\sim 6 \mathrm{R}_{\mathrm{J}}$, liberating $\sim 10^{3} \mathrm{~kg} \mathrm{~s}^{-1}$ of sulphur and oxygen plasma. This plasma is shown by the dotted region, which rotates rapidly with the planetary field due to magnetosphere-ionosphere coupling while more slowly diffusing outwards. Three separate angular velocities associated with this coupling are indicated. These are the angular velocity of the planet $\Omega_{J}$, the angular velocity of a particular shell of field lines $\omega$, and the angular velocity of the neutral upper atmosphere in the Pedersen layer of the ionosphere, $\Omega_{J}^{*}$. The latter is expected to lie between $\omega$ and $\Omega_{J}$ because of the frictional torque on the atmosphere due to ion-neutral collisions. The oppositely directed frictional torque on the magnetospheric flux tubes is communicated by the current system indicated by the arrowed dashed lines, shown here for the case of sub-corotation of the plasma (i.e. $\omega \leq \Omega_{J}$ ). This current system bends the field lines out of meridian planes, associated with azimuthal field components $B_{\phi}$ as shown

where the symbols have the following meanings: $L$ denotes the equatorial crossing distance of the flux tube in units of $\mathrm{R}_{\mathrm{J}}$, the planetary radius; $\omega_{J}$ is Jupiter's angular velocity of rotation; disc plasma along the flux tube extending to $L$ rotates with angular velocity $\omega_{J}-\delta \omega$; $\Sigma$ is the height-integrated ionospheric Pedersen conductivity; $\dot{M}$ is the rate at which new plasma mass is added to the disc at its inner boundary, the Io torus; and $\mathrm{B}_{\mathrm{J}}$ is the equatorial field strength at the planet's surface.

This equation clearly indicates the important role played by the ionospheric conductance and the mass loading rate of Iogenic plasma. For increasing $\Sigma$ and decreasing $\dot{M}$, Hill (1979) showed that the 'critical' value of $L$, beyond which the plasma subcorotates to a significant degree $\left(\delta \omega / \omega_{J} \gtrsim 0.3\right)$, would increase. This tells us that the plasma is more efficiently accelerated towards corotation in systems with highly conducting ionospheres and/or low plasma mass loading rates. Mathematically, the value of $L$ at which this level of breakdown in corotation arises is given by:

$$
L_{0}=\left(\frac{\pi \Sigma \mathrm{B}_{\mathrm{J}}^{2} \mathrm{R}_{\mathrm{J}}^{2}}{\dot{M}}\right)^{1 / 4}
$$

When we substitute appropriate values of $\Sigma=0.2$ mho (e.g. Cowley and Bunce 2001), $\dot{M}=1000 \mathrm{~kg} \mathrm{~s}^{-1}$ (Bagenal and Delamere 2011), and $\mathrm{B}_{\mathrm{J}}=428000 \mathrm{nT}$, we obtain a value $L_{0} \sim 28 \mathrm{R}_{\mathrm{J}}$. Additional variants of Hill's theory have included the effects of non-dipolar fields, global changes in magnetospheric configuration and precipitationinduced enhancement of the ionospheric conductance. (e.g. Cowley and Bunce 2001; Nichols and Cowley 2004; Cowley et al. 2007). Other theoretical studies of the magnetosphere-ionosphere coupling have included more realistic, global-circulation models for the thermosphere-ionosphere (e.g. Achilleos et al. 2001; Smith and Aylward 2009; Yates et al. 2012). The most recent study of this kind (Yates et al. 2012) found that the 
total energy transferred from the planet's rotation to the atmosphere and magnetosphere varies with magnetodisc size. The total power dissipated in the coupled system changes from $\sim 400 \mathrm{TW}$ to $\sim 700 \mathrm{TW}$ as the plasmadisc radius expands from $45 \mathrm{R}_{\mathrm{J}}$ to $85 \mathrm{R}_{\mathrm{J}}$. This prediction is consistent with the theoretical work of Southwood and Kivelson (2001), who demonstrated that the steady-state intensity of the auroral currents should be higher for a more expanded magnetosphere, characterised by a plasmadisc which rotates more slowly.

The addition of newly ionised plasma in the vicinity of the Io torus represents a momentum 'loading' for the system. Freshly created ions initially move at the Keplerian velocity of the neutral molecules from which they formed, and must then be picked up or incorporated into the ambient, corotating plasma flow-this requires an increase in the angular momentum of the newly ionised plasma, which is ultimately provided by the deep layers of the rotating planet. The processes of ion pickup and radial plasma transport both slow the magnetospheric plasma below rigid corotation, extract angular momentum from the planet, and produce magnetosphere-ionosphere coupling currents. However, there is also a process which can change the local angular momentum of the disc plasma, without adding additional plasma mass. This is the phenomenon of charge exchange which may occur between rapidly rotating ions and slower, cold neutrals (e.g. on Keplerian orbits) in a magnetosphere where the neutral-to-ion ratio is considerably high, such as that of Saturn. The transfer of electrons from the cold neutral to the hot ion results in the formation of a cold ion and a hot neutral, the latter now free from the influence of the magnetic field and thus capable of carrying energy away and escaping from the magnetosphere entirely.

Delamere et al. (2007) modelled the energy and particle flows for the Io and Enceladus tori, incorporating the effects of ion pickup and charge exchange (see Sect. 2 for more details). They concluded that the relative importance of charge exchange and radial transport of plasma was sensitive to the ratio of neutral to ion number density in the torus. At Saturn/Enceladus, where this ratio is about $\sim 12$, the net effect of charge exchange is to carry away $\sim 95 \%$ of the mass which is added to the torus per unit time (principally in the form of water group molecules, which may then become ionised). The remaining $\sim 5 \%$ of this material is transported radially outwards as plasma. At Jupiter/Io, on the other hand, neutral species in the torus model (mainly sulphur and oxygen) have number densities about $\sim 1 \%$ of their corresponding ion products. Under these conditions in the Jovian system, charge exchange is predicted to remove about half of the mass added to the Io torus per unit time. This result demonstrates the more dominant role played by radial plasma transport in the overall mass flow of the Jovian system.

In a related study, Pontius and Hill (2009) developed an equation of motion for the disc plasma at Saturn which included the effects of ion-neutral charge exchange. They then used this theory to 'invert' observations of plasma angular velocity by Cassini, and demonstrated that the charge exchange process was the major contributor to decreasing the angular momentum of the plasma within regions $L \lesssim 5$. At $L=3.4-3.8$ in Pontius and Hill's (2009) modelling study, for example, they show that the charge exchange process, in terms of momentum loading, is equivalent to adding $\sim 350 \mathrm{~kg} \mathrm{~s}^{-1}$ of plasma to the disc. In this same interval of distance, the mass loading rate due to actual, newly ionised plasma (i.e. a genuine increase in plasma mass) is $\sim 8 \mathrm{~kg} \mathrm{~s}^{-1}$. The same authors estimate a total outward mass flux of $\sim 100 \mathrm{~kg} \mathrm{~s}^{-1}$ near $L=7.5$, for an ionospheric height-integrated conductivity of 0.1 mho.

The aforementioned studies suggest, then, that radial transport of plasma plays a major role in the evolution of the Jovian magnetodisc, at least. The question then arises as to the nature of this transport process: How do we move disc plasma in the radial direction without eventually 'breaking' the magnetic flux tubes to which this material is 'frozen in'? An important clue arises from in situ observations of the magnetic field in the vicinity of the 
plasma tori themselves. On spatial scales which are large compared with Io and Enceladus, the magnetic field near the corresponding tori is always well approximated by a rigid, dipolar configuration, arising mainly from the planet's internal dynamo. Indeed, the model results from Fig. 1 reveal that the $L<10$ region of Jupiter's magnetosphere is characterised by a magnetic energy density more than ten times the bulk kinetic energy of the rotating plasma. Hence, the Io torus is clearly inside a region where we expect the magnetic field to strongly resist any large-scale 'deformation' due to plasma flow. The radial transport mode required should thus be able to displace plasma mass outwards from its torus of origin, without any attendant displacement of magnetic flux. The most widely proposed transport mechanism which satisfies these requirements is that of flux tube interchange, a process which, in some sense, relies on the development of a small-scale 'texture' in the disc plasma.

In this chapter, we limit our discussion of plasma transport to the interchange process within the magnetodisc region, which arises from a fluid instability. In a more general context, other forms of radial plasma transport may arise when fluctuations of magnetic or electric fields occur. If the fluctuation time scale is larger than a particle bounce period, but small compared to the time scale of azimuthal drift, the first two adiabatic invariants may be conserved, but not the third. Hence particles will radially 'diffuse' (e.g. Brice and McDonough 1973; Walt 1994; Schulz 1979). If field fluctuations occur on adequately small time scales compared to that of bounce motion, the first two adiabatic invariants will not be conserved and particle energy and equatorial pitch angle will also be subject to diffusion. Such rapid fluctuations may be associated with plasma waves, and the interaction of these waves with particles can be the basis for this type of diffusion (e.g. Walt 1994; Thorne 2010).

Sections 4 and 5 discuss more recent, advanced models for the interchange process at both Jupiter and Saturn which incorporate additional effects such as pressure anisotropy and multi-fluid magnetohydrodynamics. However, we may start to introduce the concept here, in a simple way, by appealing to the early description of Gold (1959). He compared the nature of a putative flux-tube-interchange process in the Earth's magnetosphere with that of convection in a gravitating, compressible fluid. In the latter case, consider the temperature gradient $-\nabla T$ in the fluid, whose direction (from higher to lower temperature) opposes gravity. If the magnitude of this gradient is above a critical value, known as the adiabatic temperature gradient, the gas becomes unstable to convection. The critical driver for the process is the fact that, for adequately steep gradient in temperature, the convective mixing of parcels of gas will lead to a lower total energy for the new fluid configuration.

Applying this reasoning to a simple model of interchange, Gold (1959) considered the outward displacement of a single plasma flux tube in a rigid, dipole magnetic field (a compensating inward displacement of a plasma tube at larger radial distance maintains the largescale, dipole configuration of the field). As the flux tube moves outward, its equatorial cross section intercepts a constant increment in magnetic flux, and its volume $V$ changes in proportion to the quantity $L^{4}$. In the limiting case of adiabatic transport, the pressure $P$ of the plasma in the tube would thus change according to $P \propto L^{-4 \gamma}$, where $\gamma$ is the ratio of specific heats for the plasma (e.g. $\gamma=\frac{5}{3}$ for an ideal, monatomic gas). This 'adiabatic gradient' for pressure thus plays an analogous role to the adiabatic temperature gradient for the gravitating fluid.

Hill (1976) extended this idea to a rapidly rotating model magnetosphere, specifically in order to analyse the properties of the Jovian system. He considered the change in centrifugal potential energy of plasma which would result from the exchange in location of two flux tubes embedded in a pure dipole field. For a system where the flux tube content $M_{B}$ (mass of plasma contained in a tube whose cross section encloses unit magnetic flux) decreases 
monotonically with distance, Hill (1976) supposed that the system would be unstable to such interchange motions when they led to a decrease in the total centrifugal potential, and he showed that this would happen when the following condition was satisfied:

$$
\frac{\partial M_{B}}{\partial r_{e}}<0
$$

In other words, a monotonic decrease in flux tube content $M_{B}$ with equatorial crossing distance $r_{e}$ encourages interchange motions in a system whose dominant energy arises from bulk rotation. This description is appropriate for the cold, rapidly rotating plasma within the Jovian magnetodisc. It is also important to note that the result of interchange motions is to reduce the spatial gradient in flux tube content, and hence drive the system towards a lowerenergy, more stable state. The continual addition of Iogenic plasma to the disc ensures that this stable state is never reached and that interchange continually acts to move newly created plasma radially outwards.

Southwood and Kivelson (1989) further extended the theoretical treatment of interchange motion, and quantified the effects of the interaction between the moving flux tubes and the planetary ionosphere. Specifically, they showed that the timescale for growth of interchange motions increases linearly with ionospheric Pedersen conductance $\Sigma_{P}$. This result reflects the 'damping' effect which arises when a highly conducting ionosphere transmits a retarding force to flux tubes participating in interchange motion. As with the large-scale rotational motions of the disc plasma described above, this conveyance of momentum is achieved by field-aligned currents, which travel along the flanks of the moving tubes and close in the ionosphere. The ionospheric currents are associated with ion-neutral collisions between the ionospheric plasma and the planet's thermosphere. The transfer of momentum due to these collisions is the ultimate source of the retarding force on the moving flux tubes. Southwood and Kivelson (1989) favoured a diffusion-like mechanism to maintain the strong inward gradient of the cold plasma's flux tube content at the outer edge of the Io torus (i.e. tube content strongly decreases with increasing radial distance). Beyond this region, their depiction of the most likely nature of the plasma flow involved interchange motions whose combined effect, over long time scales, was to migrate regions of high flux tube content outwards, and depressed flux tube content inwards. For a temporally variable Io plasma source, the associated flow streamlines would be continually changing in a random manner. The local motion of a flux tube (inward or outward) in this picture is determined according to whether it has an elevated or depressed plasma content compared to the average content of its surroundings.

More than three decades after Gold's analysis (Gold 1959) of the interchange process, the Galileo orbiter at Jupiter gave scientists the opportunity to search for observational signatures of this mode of plasma transport. Kivelson et al. (1997b) reported short-duration increases in the magnetic field strength, of typical amplitude 1-2\% of the background field and average duration $\sim 26 \mathrm{~s}$. These events were observed over an equatorial distance range $\sim 6-7.7 \mathrm{R}_{\mathrm{J}}$. Although the change in field strength was typically small, the corresponding change in magnetic pressure was often comparable to the background plasma pressure. One particular event at $6.03 \mathrm{R}_{\mathrm{J}}$ was analysed in detail by Kivelson et al. (1997b), and was consistent with an inward-travelling flux tube of depleted plasma density, equal to $\lesssim 50 \%$ of the background (outside the tube) value. The distance of origin for this depleted tube was estimated to be $7.2 \mathrm{R}_{\mathrm{J}}$, the location where the flux tube content of the background plasma would be equal to that inside the inward-travelling tube.

The absence of ion-cyclotron wave signatures inside the depleted tube was interpreted as evidence that it had not existed for a long enough period in the near-Io plasma environment for these waves (themselves signatures of ion pickup) to appreciably grow. Indeed, 


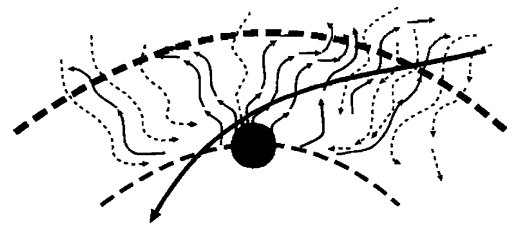

Fig. 3 Schematic of the plasma transport near the Io torus from Kivelson et al. (1997b). Dashed arcs denote the orbit of Io, and $7 \mathrm{R}_{\mathrm{J}}$. Other meandering curves represent averaged, rather than instantaneous, flows. Solid (dashed) curves of this type indicate outward (inward) moving flux tubes. Well away from Io, inward and outward flows balance. The vicinity of Io itself (filled circle) is a region dominated by mass loading, and thus predominantly outward flow. Inward flow dominates elsewhere, presumably. The inbound Galileo trajectory is shown as a solid, curved arrow. The spacecraft crosses both inward- and outward-moving plasma near $7 \mathrm{R}_{\mathrm{J}}$, but mainly outward-moving material nearer to Io

Huddleston et al. (1997) applied this argument to estimate a radial velocity of travel for the depleted tube around $100 \mathrm{~km} \mathrm{~s}^{-1}$. A more advanced description of the wave signatures associated with mass loading in the magnetodisc will be given in Sect. 6. Analysis of the same field enhancement event by Thorne et al. (1997), incorporating plasma wave data, indicated that the depleted plasma density could have been as low as $\sim 3 \%$ of the background value, thus suggestive of a strong contribution by energetic particles to the plasma pressure. These Galileo studies were consistent with a scenario in which heavier flux tubes (i.e. higher plasma content than background) are outward-moving, and outward motions are dominant close to Io itself. They are presumably balanced by inward motions at other longitudes. At larger distances, inward-moving, depleted tubes are observed, as described above. In Fig. 3, we show the schematic diagram from Kivelson et al. (1997b) which illustrates these aspects.

Similar observational signatures of the centrifugally driven interchange process have been observed by Cassini at Saturn. Rymer et al. (2009) reported a short-duration field strength enhancement of amplitude $\sim 6 \%$ of the background field, corresponding to an electron density about one third of the background value. The particle energy spectrogram acquired by the Cassini plasma spectrometer (Young et al. 2004) exhibited the characteristic dispersions associated with the energy-dependent particle drifts that would presumably occur during the inward motion of the depleted tube. The data were consistent with a tube that had moved inward from $\sim 10 \mathrm{R}_{\mathrm{S}}$ to $7 \mathrm{R}_{\mathrm{S}}$ at a radial speed of $150-300 \mathrm{~km} \mathrm{~s}^{-1}$, of the same order as the Jovian observations. Earlier observational studies at Saturn (e.g. Hill et al. 2005; Chen and Hill 2008) analysed what they referred to as plasma injection signatures over an equatorial distance range $\sim 6-11 \mathrm{R}_{\mathrm{S}}$. The azimuthal extent of injection events with electron energies 1-30 keV were estimated to be of the order $\lesssim 1 R_{S}$, and to survive less than one planetary rotation.

Moving now into the outer reaches of the 'magnetodisc' region and beyond, the nature of plasma transport is observed to change. Generally speaking, the relatively small perturbations to the background plasma which describe the interchange process are replaced in the outer magnetosphere by much larger-scale injections of plasma which is usually more energetic and tenuous than the disc plasma. Mauk et al. $(1999,2002)$ quantified this transition in the Jovian system through analysis of Galileo particle data. They described a change in the 'character' of injections beyond distances of $\sim 9 \mathrm{R}_{\mathrm{J}}$. Specifically, the injections in the more distant magnetosphere are associated with increased fluxes of energetic particles, and more pronounced energy dispersion signatures, even evident in electrons. Mauk et al. (1999) analysed more than 100 events over the distance range 9-27 $\mathrm{R}_{\mathrm{J}}$, which occurred over a wide range in longitude and local time. This property of the Jovian plasma injections highlights 
the contrast with similar events in the Earth's magnetosphere, whose local time distribution suggests a clustering around the dawn sector, consistent with a physical origin in the terrestrial magnetotail.

Mauk et al. (1999) also found that a typical large-scale plasma injection in their sample moved a few $\mathrm{R}_{\mathrm{J}}$ before it became significantly dispersed through differential drift motions. About five injections per day were observed by Galileo under quiescent magnetospheric conditions. This rate rose to approximately 30 events per day, following global magnetospheric disturbances or reconfigurations. The typical azimuthal extent of an injection was $\sim 30-40^{\circ}$, in contrast with the few degrees for the interchange-related events described above. In a related study, Louarn et al. (2001) examined the correlation between Galileo plasma data, and radio data relating to regions in the outer Io torus. Their results suggested that relatively young, 'dispersion-free' injections were signatures of an 'unloading' process, which acted quasi-periodically to remove plasma from an active region in the torus. This phenomenon may be related to the 'energetic events' described by Louarn et al. (1998) that trigger sequences of plasma loading and unloading in the magnetodisc (Louarn et al. 2000). The existence of these large-scale, energetic plasma injections in the Jovian system naturally raises the question of whether they and the narrower 'interchange tubes' are in fact different signatures of a unified, global process of plasma transport. At present, we do not have a definitive answer.

The large-scale variety of plasma injections have also been observed in Saturn's magnetosphere. Injections with particle energies in excess of $\sim 100 \mathrm{keV}$ have been observed, and inferred to have survived up to several Saturn rotations from their time of formation (Mauk et al. 2005; Paranicas et al. 2007). Plasma injections extending to tens of degrees in azimuth have been remotely observed through energetic neutral atom (ENA) imaging (Krimigis et al. 2005; Carbary et al. 2007). It has been suggested that these may originate in the planet's magnetotail, and rotate more quickly as they move inwards. ENA injections have also been convincingly linked to rotating, arc-like auroral emissions at Saturn, to which they are likely to be magnetically conjugate (Mitchell et al. 2009). Section 3 describes these injections and their analysis in more detail.

After its creation and radial migration through a disc-like region, the final stage of the transport of magnetospheric plasma is, of course, its final release from its parent planetary system. One of the key phenomena which underpins this process is the fact that outwardmoving flux tubes cannot maintain their integrity indefinitely. The continual process of internal mass loading (associated with satellite tori) leads to the plasma sheet thickening in the dusk sector, and the strong radial expansion of flux tubes as they rotate into the magnetotail region, now unencumbered by the proximity of the magnetopause (e.g. Kivelson and Southwood 2005).

A well-known scenario for mass loss in this context is the intermittent release of plasma from these highly distended and mass-loaded flux tubes, a process which was first discussed by Vasyliūnas (1983). In this model, the corresponding part of the plasma sheet becomes unstable once these outer flux tubes carry a critical amount of plasma and rotate past the dusk sector into the magnetotail. Without the 'bracing' of a nearby magnetopause boundary to stabilise it, the plasma sheet becomes disrupted and sheds individual parcels of plasma, or plasmoids. A more detailed discussion of this process and magnetic reconnection in the magnetotail region is given in other chapters of this volume. For present purposes, we note that planetary rotation at the gas giant systems is also thought to influence the stability of these heavily loaded flux tubes. Kivelson and Southwood (2005) pointed out that combining concepts from magnetohydrodynamic and kinetic theories were important for understanding the dynamics of plasma embedded in these structures. Specifically, they considered the 
motion of a heavy (20 proton masses), corotating ion with thermal energy small compared to its rotational energy. Such an ion would gain $\sim 20 \mathrm{keV}$ in energy parallel to the field as it moves from 45 to $50 \mathrm{R}_{\mathrm{J}}$ in cylindrical radial distance. If such ions acquire an adequate amount of energy parallel to the field direction, then the plasma at the equatorial portion of the flux tube will become subject to a firehose-type instability, and the magnetic field will not be adequately strong to constrain the rapid, field-aligned motion of the plasma. Consequently, the plasma sheet becomes unstable, distending and thinning, which leads to the loss of plasma from the outer edge of the disc and the return of depleted, closed flux tubes towards the planet.

In summary, then, we have found that the rapid planetary rotation associated with the gas giant systems has a fundamental influence on the transport of magnetospheric plasma, and the communication of energy and momentum between the magnetodiscs and the ionospheres of these worlds. To improve our understanding of both processes, several advancements are required, from both observational and theoretical points of view. We list some of these here:

- Additional surveys of the plasma angular velocity, over comprehensive ranges in distance and in local time.

- More theoretical work coupling global-circulation models of planetary thermospheres with plasma flow models for magnetospheres. Particularly relevant in this context would be the further exploration of time-dependent effects of magnetospheric reconfigurations on the auroral currents, and further work with three-dimensional models, in order to explore local time effects.

- The further development of an observational 'interchange budget', based on the statistics of plasma injection events, as well as the comparison of different injections' spatial scales, dispersion characteristics, and correlations with near-coincident magnetospheric reconfigurations.

- Further refinement of the theory of flux tube interchange, considering, for example, the shapes and scales of the underlying, small 'overturn motions' which drive the global plasma migration. Another major topic to address is the possibly inter-related influence on interchange of: (i) dramatic changes in the rate of internal mass-loading; and (ii) the external driving of the magnetospheric field structure by transient changes in the solar wind dynamic pressure and/or the interplanetary magnetic field.

Finally, we conclude this section with a summary of some of the outstanding questions relating to magnetodisc structure and dynamics (some of the following sections also have summaries related to their relevant topics):

- What is the range of timescales for the localised motions, or 'interchange overturns', which support the global radial transport of plasma?

- Do we actually detect interchange signatures with adequate spatial coverage and frequency in order to explain the global rates of plasma transport which are required, on average, at both the Jovian and Kronian magnetospheres?

- How do the larger-scale injection events contribute to global radial transport, and how do they relate to the smaller-scale, centrifugally driven interchange events?

- Does the outward-moving, colder plasma actually extend to very large azimuthal distances between the hotter, more tenuous flux tubes, and is this why its observational signatures are lacking by comparison?

- How do anisotropy forces, for both hot and cold plasma, affect the global structure of the disc and the higher-latitude plasma?

- What are the acceleration mechanisms responsible for establishing the anisotropy in the hot plasma pressure? 
Fig. 4 Saturn's E-ring of neutral gas formed near the orbit of Enceladus from water vapor vented from its south pole plumes. (Image credit: NASA/JPL/Space Science Institute)

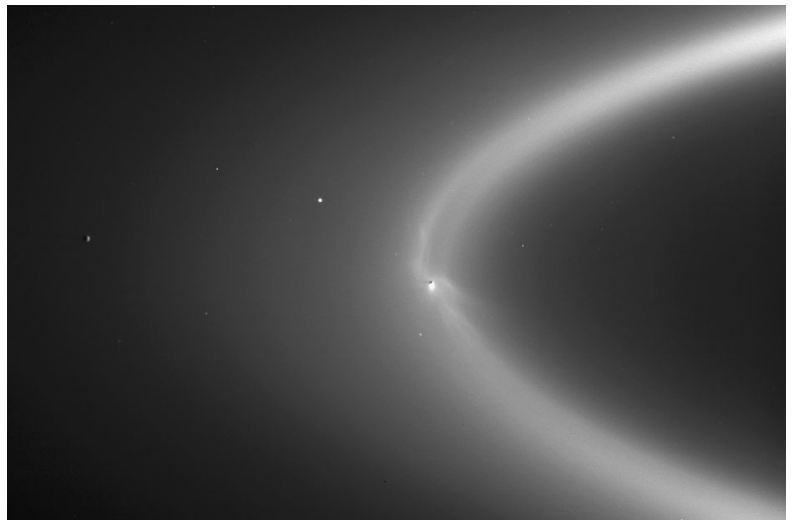

\section{The Neutral-Plasma Interaction}

\subsection{Introduction}

The giant planet magnetodiscs are supplied by mass from satellites orbiting deep within the magnetosphere. At Jupiter, Io revolves around the gas giant with an orbital distance of $6 \mathrm{R}_{\mathrm{J}}$ and supplies the inner magnetosphere with roughly 1 ton per second of neutral sulfur and oxygen from its prodigious volcanic activity (Thomas et al. 2004). At Saturn, Enceladus orbits at a distance of $4 \mathrm{R}_{\mathrm{S}}$ and vents roughly $200 \mathrm{~kg} \mathrm{~s}^{-1}$ of water molecules from geysers at its south pole to form Saturn's E-ring (Mauk et al. 2009). The distances to the respective magnetopause boundaries are roughly $75 R_{J}$ (Joy et al. 2002) and $24 R_{S}$ (Kanani et al. 2010), placing these satellites deep within the inner magnetosphere. The fate of these neutral gases are very different when comparing Jupiter with Saturn. Jupiter's inner magnetosphere is dominated by plasma as most of the neutral gas from Io is ionized (Delamere et al. 2007). Saturn's inner magnetosphere remains neutral dominated due to very low ionization rates (Delamere and Bagenal 2008). This section will compare and contrast the evolution of neutral gas and transport of plasma through the giant planet magnetodiscs.

Escaping neutral gases typically have speeds that are not significantly different from the Keplerian velocity of the parent satellite. For example, the escape velocity from Io is roughly $2.5 \mathrm{~km} \mathrm{~s}^{-1}$, while Io's Keplerian velocity is roughly $17 \mathrm{~km} \mathrm{~s}^{-1}$. As a result the neutral gases will tend to form a torus of gas about the orbit of the satellite (Fig. 4). However, if ionization occurs then the evolution of the gas clouds is altered significantly.

There are essentially three neutral gas loss mechanisms to consider: 1) photo ionization, 2) electron impact ionization by a thermal background plasma, and 3) charge exchange (Delamere and Bagenal 2003; Fleshman et al. 2010a). Other processes such as electron impact dissociation and photodissociation of molecular species and neutral-neutral collisions can scatter neutral particles resulting in loss to the planet or rings; however, as loss mechanism, these scattering processes are relatively minor (Fleshman et al. 2012; Cassidy and Johnson 2010; Farmer 2009; Johnson et al. 2006). Photoionization timescales at 5 and 9 AU are typically of order of months to years. Compared to the orbital period for Keplerian motion (days) and the spreading of the neutral gas due to small difference in Keplerian speeds (tens of days), a complete neutral torus would be expected to form in the absence of electron impact ionization mechanisms. If, however, electron impact ionization 
Fig. 5 An example of Io's partial neutral oxygen torus from Burger (2003)

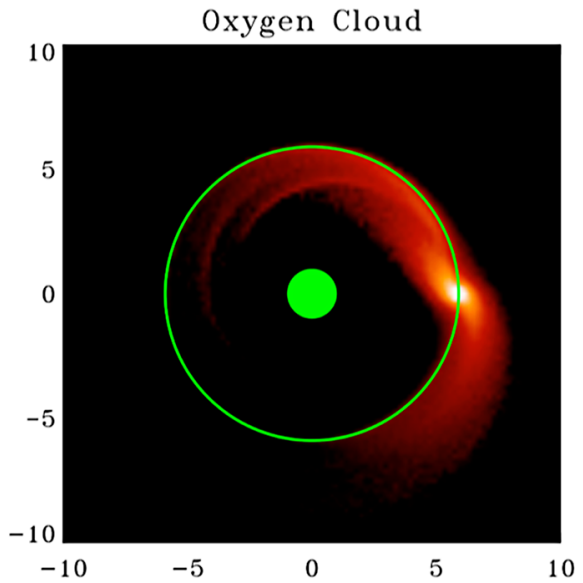

is faster than $\approx$ tens of days, then a partial neutral torus will form due to relatively prompt losses of the neutral gas cloud.

The ionization energy for most neutral species ranges between 10 and $15 \mathrm{eV}$. For a Maxwellian distribution, significant ionization can still be achieved at lower temperatures via the high energy tail of the distribution. The ionization rates for sulfur (i.e. $\mathrm{S}$ to $\mathrm{S}^{+}$) are sufficient to erode the sulfur neutral clouds extending from Io such that only a partial neutral torus forms. While no global observation of Io's neutral torus has been made (see Brown 1981; Skinner and Durrance 1986 for examples of measurements far from Io inferred from line-of-sight UV observations), model results show that the torus is indeed partial (Smyth and Marconi 2003; Burger 2003) as shown in Fig. 5.

The thermal electron population in the Io plasma torus is observed to be roughly $5 \mathrm{eV}$ (Sittler et al. 2007), while the electron temperature in the Enceladus E-ring region is less then $2 \mathrm{eV}$ (Young et al. 2004; Schippers et al. 2008). The electron impact ionization rates increase by roughly two orders of magnitude between $2 \mathrm{eV}$ and $5 \mathrm{eV}$. This accounts, in part, for Jupiter's plasma-dominated inner magnetosphere and Saturn's neutral-dominated inner magnetosphere (Delamere et al. 2007). The ubiquitous hot electrons, observed in both systems, can also provide ionization, but as we will discuss below, the hot electrons at Jupiter serve to heat the thermal electrons and increase the ionization state (i.e. average charge of the ions) of the plasma torus. At Saturn, the hot electrons provide ionization at a level that is comparable to photoionization but due to the tenuous plasma, cannot provide significant energy via Coulomb interactions to the thermal electrons.

Additional chemistry must be considered to fully understand the evolution of the neutral clouds and ultimate fate of these gas particles. Charge exchange between ions and neutrals results in high-speed neutrals that can potentially escape from the system completely, or force collisions with the planet or ring systems. This mechanism leads to the permanent loss of neutral material from the system and cannot further participate in the plasma mass loading of the magnetosphere (Cassidy and Johnson 2010; Fleshman et al. 2012). Other reactions involve molecular species that can dissociate via electron impact into smaller molecules or atomic species. Molecular ions likewise can suffer electron impact dissociation, yielding smaller neutral fragments that can likewise escape from the system. Photodissociation for molecular species can also be important (Fleshman et al. 2010a).

In this section we will discuss the mass loading of the inner magnetosphere and the flow of energy and mass through the system. 


\subsection{Momentum Loading and Neutral Cloud Evolution}

Ionization and charge exchange both involve momentum loading of the magnetosphere. The Keplerian speed of neutral particles is significantly less than the local plasma flows that are typically a significant fraction of the corotation speed of the planet. At Jupiter and at Io's orbit, the corotation speed is roughly $74 \mathrm{~km} \mathrm{~s}^{-1}$, and the difference between the Keplerian speed and the corotation speed is $57 \mathrm{~km} \mathrm{~s}^{-1}$. This means that new ions will be picked up (from Keplerian speed) to the local flow speed (corotation speed), requiring a transfer of momentum from the planet to the new ions. Mass loading the plasma flow results in a slowing (subcorotation) of the flow and the related magnetic field perturbations will couple (via an Alfvénic perturbation/field-aligned currents) the mass-loaded plasma to the planetary ionosphere. In steady state, the "pickup current" results from the $\mathbf{J} \times \mathbf{B}$ force that balances the momentum loading, specifically when $\dot{M} v_{\text {plasma }}=\int \mathbf{J} \times \mathbf{B} d V$, integrated over the mass loaded volume (see Vasyliūnas 2006 for a detailed discussion on the physical origin of the "pickup current").

Alternatively, mass loading and ion pickup can be understood by considering the rest frame of the neutrals. In this frame, the plasma is flowing past the stationary neutrals and has an associated convection electric field, $\mathbf{E}=-\mathbf{v}_{\text {plasma }} \times \mathbf{B}$. In this case, the new ion is accelerated by the convection electric field and acquires an average drift velocity equal to the local flow speed and in the flow direction together with a gyromotion with a speed that is also equal to the local flow speed. The combination gives a cycloid motion as illustrated in Fig. 6. The resulting velocity distribution is a two-dimensional ring beam (perpendicular to the local magnetic field) with temperature, $T_{\perp}=\frac{1}{2} m v_{\text {plasma }}^{2}$. Typically, this temperature is tens to hundreds $\mathrm{eV}$. This unstable distribution can generate electromagnetic ion cyclotron (EMIC) waves which scatter the ring beam toward an equilibrium Maxwellian distribution (Blanco-Cano et al. 2001c; Russell and Huddleston 2000). However, the scattering time scales and the mass loading time scales can be comparable leading to the observed temperature anisotropies $\left(T_{\perp} / T_{\|}>1\right)$ in both Io (Crary et al. 1996) and Enceladus plasma tori (Sittler et al. 2008). Figure 7 illustrates the combined velocity distribution of a ring beam (blue) embedded in a cooler Maxwellian distribution (red).

A critical issue, however, is that the electrons produced during ionization are picked up with an energy that is much less than the ionization potential of the neutral gas (i.e. $\ll 10 \mathrm{eV}$ ). Only through Coulomb coupling to the ions and superthermal electrons will the thermal electrons attain a sufficient energy to ionize. But the effectiveness of the Coulomb coupling is dependent on the plasma density and this energy source can also compete with radiative cooling losses. For a complete discussion of this energy flow, see discussions by Shemansky (1988) and Delamere et al. (2007).

Both ionization and charge exchange momentum load the local plasma flow, but generally only ionization adds mass to the system. Charge exchange reactions can liberate a fast neutral with speeds $\sim v_{\text {plasma }}$, in principle, at any point of the cycloid trajectory. These fast neutrals will generally leave the system (i.e. escape from the magnetosphere). However, Fleshman et al. (2012) note that charge exchange cross sections are generally velocity dependent and in the case of symmetric reactions, the cross sections are a strong function of velocity with large increases at low velocity. If the ions move in perfect cycloids, then the reaction rates increase significantly near the cycloid cusps where the relative velocity between the ion and neutral is minimized. The slow moving neutral products will thus linger in the inner magnetosphere and the slow velocity charge exchange reaction can be considered as an important mechanism for spreading the neutral clouds. Other mechanisms for spreading the neutral clouds include dissociation of molecular species due to the residual kinetic energy distribution of the dissociated products (typically $1 \mathrm{~km} \mathrm{~s}^{-1}$ ) (Fleshman et al. 2012). 
Fig. 6 An illustration of the neutral-plasma interactions near Io. Incident plasma interacting with Io's atmosphere lead to ionization, charge exchange and induced dipole interactions that collectively lead to an atmospheric sputtering process at or near the exobase. (Image credit: F. Bagenal/S. Bartlett)

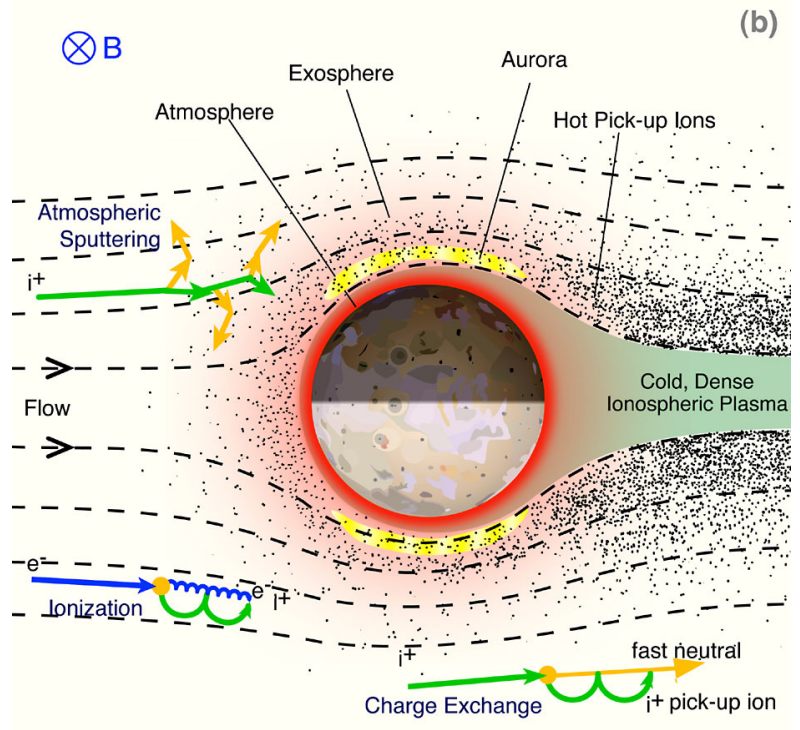

Fig. 7 An illustration of a ring beam distribution (blue) combined with a cooler Maxwellian distribution (red) near Io

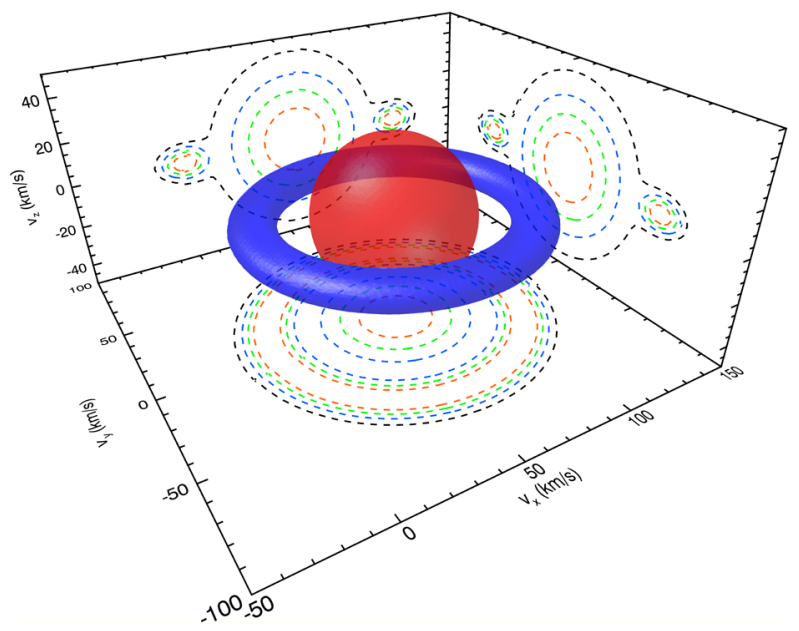

\subsection{Satellite-Magnetosphere Interactions}

At the root of neutral-plasma interactions at Jupiter is the source of neutral material from Io's atmosphere. Io's atmosphere is created through the direct injection of gases from volcanic vents and through sublimation of the surface frosts originating from volcanic plume fallout. The relative contribution from these processes has generated significant debate (see reviews by McGrath et al. 2004; Lellouch et al. 2007). The atmosphere exhibits day/night asymmetries, supporting the sublimation source, yet the persistence of atmospheric emissions during eclipse supports a direct volcanic source. Io's atmosphere is confined to its equatorial regions and appears to contain multiple components that include a tightly bound $\mathrm{SO}_{2}$ and $\mathrm{SO}$ atmosphere, together with an extended corona of atomic $\mathrm{S}$ and $\mathrm{O}$ species. The 
corona is also an expected result of an escaping atmosphere, namely the 1 ton per second of material that escapes from Io to populate the extended neutral clouds.

Various approaches have been adopted to model Io's interaction with Jupiter's magnetosphere. Early models adopted a fully three-dimensional, MHD approach where Io's surface used prescribed boundary conditions (e.g. finite conductivity) and a distributed mass loading rate to represent ionization and charge exchange (Combi et al. 1998; Linker et al. 1998; Kabin et al. 2001; Khurana et al. 2011). Saur et al. (1999) used a two-fluid model to model the flow around a conducting atmosphere, solving for the electric fields and currents associated with the interaction. However, these authors did not self-consistently solve for the magnetic field perturbation, but rather determined the perturbation based on the modeled currents. A hybrid simulation was also attempted by Lipatov and Combi (2006) to improve the treatment of mass loading and the related generation of non-Maxwellian pickup distributions. More recently, Dols et al. (2012) used a MHD approach, coupled with mass loading rates determined from a physical chemistry model of the local Io interaction (Dols et al. 2008). The coupled approach, combined with comparisons with data from the various Galileo flybys, led Dols et al. (2012) to suggest that Io's outer atmosphere/corona is not distributed uniformly in longitude. Instead, it is extended in the wake direction and perhaps even more extended in the anti-Jupiter direction.

There is one possible explanation worth mentioning for a possible asymmetric atmosphere. Using magnetic field perturbations seen in the Galileo magnetometer data, Russell et al. (2003b) concluded that an asymmetric distribution of ion cyclotron wave power could be generated if charge exchange preferentially redistributes the neutral atmosphere to the wakeward and anti-Jupiter directions because ion pickup motion from charge exchange reactions is initially in this direction. Subsequent ionization of these scattered neutrals will lead to the generation of EMIC waves. Crary and Bagenal (2000) used the ion cyclotron waves as a constraint on density and escape rate of the neutral atmosphere. These initial assessments of Io's atmosphere are consistent with the subsequent analysis of Dols et al. (2012).

Roughly $10^{12} \mathrm{~W}$ of power is generated by Io's interaction with the plasma torus (Saur et al. 2004; Hess et al. 2010). The escape velocity, $v_{e s c}$, from Io's surface is $\sim 2.5 \mathrm{~km} \mathrm{~s}^{-1}$. If 1 ton of neutral material escapes per second from the atmosphere, then the power associated with neutral escape $\left(\frac{1}{2} \dot{M} v_{\text {esc }}^{2} \approx 3 \times 10^{9} \mathrm{~W}\right)$ is only a small fraction $(<1 \%)$ of the interaction power. While most of the power is radiated in Alfvén waves, it is plausible that harnessing just a small fraction of this power can support Io's atmospheric escape. The escape process could involve a variety of different physical mechanisms, all of which might be considered as atmospheric sputtering. These include direct collisional heating of the neutral atmosphere via induced dipole interactions, charge exchange scattering, or even electron impact dissociation of molecular species. The latter mechanism was discussed by Dols et al. (2008) as being potentially a major contributor to the formation of Io's corona and extended neutral clouds because of substantial reaction rates ( $\sim$ tons per second) combined with a characteristic kinetic energy distribution of the dissociated products that is comparable with the escape velocity.

Finally, a potentially important aspect of the satellite-magnetosphere interaction is the feedback of field-aligned electrons. Electron beams have been observed near Io by Williams et al. (1996). Dols et al. (2008) and Saur et al. (2002) noted that these beams can interact with Io's atmosphere, providing additional ionization and heating of the atmosphere. The total power attributed to these beams from Frank and Paterson (1999) is roughly $10^{9} \mathrm{~W}$, indicating that the beams might contribute to the modification of the atmosphere via the plasma interaction. 
A study, similar to that of Dols et al. (2008) for Io, of the Enceladus plume-plasma interaction was conducted by Fleshman et al. (2010b) using a water group physical chemistry model. The tenuous plasma conditions near Enceladus do not result in a strong interaction. Typical mass loading rates, consistent with magnetic field perturbations measured near Enceladus, are small (e.g. Khurana et al. 2007 estimate a plasma mass loading rate of $\lesssim 3 \mathrm{~kg} \mathrm{~s}^{-1}$ within five Enceladus radii). Field-aligned electron beams are also present and are thought to play a more significant role in the overall chemistry associated with the Enceladus plume (Fleshman et al. 2010b). Simulation studies have investigated the role of charged dust particles in the plasma interaction and Kriegel et al. (2011) concluded that heavy negatively charged dust grains can in fact generate Hall currents in the opposite sense of currents generated by ions and electrons (with the mass of the positive and negative charge carriers reversing roles).

In summary, Io's interaction with its plasma environment results in a fascinating feedback process, modifying the atmosphere that ultimately supplies the plasma torus. Bagenal (1997) showed that most of the mass added to the torus occurs in the escaping neutral cloud rather than through local ionization of the atmosphere. Enceladus, on the other hand, already has an escaping plume of neutral gas and does not require energy input from the magnetosphere to liberate neutral gas from the gravitational well of the satellite.

\subsection{Plasma Tori}

The neutral-plasma interaction is fundamental for formation of giant planet magnetodiscs. The ultimate fate of escaping neutral gases from satellite sources is a basic problem that affects the flow of mass and energy through magnetodisc systems. Neutral gas can be absorbed by the planet, its rings, or escape from the system at high speed (i.e. greater than the escape velocity for the planet) due to charge exchange reactions. The final mass loss mechanism is via radial plasma transport and eventual loss to the solar wind (primarily down the magnetotail). In other words, the neutral source is either absorbed within the system, scattered outside the system, or lost as plasma to the solar wind. The latter mechanism has significant consequences for magnetospheric dynamics.

Bagenal and Delamere (2011) provided a comprehensive comparative study of mass and energy flow at Jupiter and Saturn. In both cases the transport time scales from satellite to solar wind is roughly tens of days. At Jupiter, roughly half of the neutral source is lost to fast neutral escape, leaving roughly $500 \mathrm{~kg} \mathrm{~s}^{-1}$ of the original 1 ton per second of gas available for plasma transport. At Saturn, Fleshman (2011) showed that only a quarter of the neutral gas survives to the plasma state. The typical neutral source from Enceladus is $200 \mathrm{~kg} \mathrm{~s}^{-1}$, indicating that only $50 \mathrm{~kg} \mathrm{~s}^{-1}$ is transported as plasma. This order of magnitude difference between Jupiter and Saturn has enormous consequences in terms of magnetosphere-ionosphere (MI) coupling. At Jupiter, the breakdown in corotation of the magnetodisc is dramatically highlighted by the main auroral oval. Saturn's auroral emissions, on the other hand, appear to map to a terrestrial-like boundary to an open polar cap with the internal plasma source not presenting the same level of mass loading to the corotating magnetosphere (Bunce et al. 2008). There has been some debate regarding the relative mass loading of these two systems, with Vasyliūnas (2008) arguing that Saturn is in fact more heavily mass loaded with Saturn's weaker magnetic field playing an important role.

Fundamentally, the plasma mass must be eventually lost to the solar wind flow. Delamere and Bagenal (2013) argued that the scale of the magnetosphere (i.e. distance to the sub-solar magnetopause) must be related to the plasma mass loss rate. At Earth the 
Fig. 8 An illustration of the scaling of Jupiter's and Saturn's magnetospheres. The vertical pink line approximates the location of the subsolar magnetopause boundary in the absence of an internal plasma source. Jupiter's magnetosphere is substantially more inflated compared with Saturn. (Image credit: F. Bagenal/S. Bartlett)

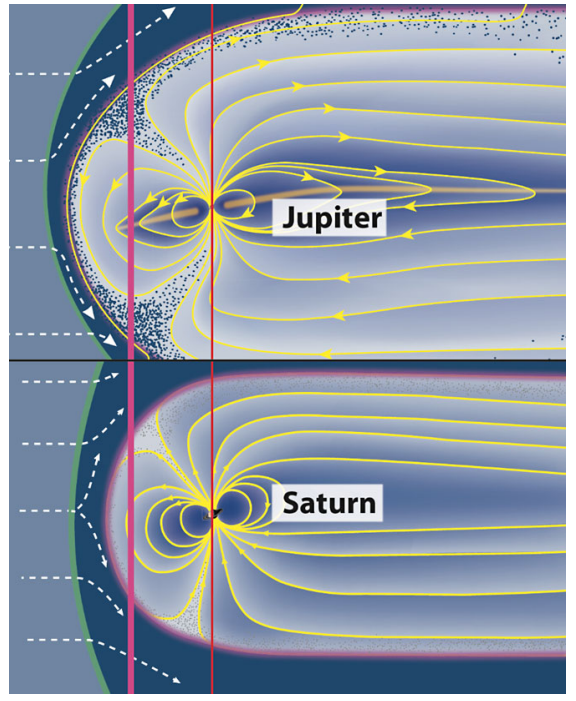

scale of the magnetosphere is determined by balancing the solar wind dynamic pressure with the planetary magnetic field pressure. This is not the case for Jupiter and Saturn where plasma pressure is an essential ingredient in the pressure balance condition. As an alternative, the momentum transfer rates from the solar wind must be able to accommodate this effective mass loading from the magnetosphere. In other words, the amount of solar wind mass per second that can be coupled via an Alfvénic interaction must balance the magnetodisc plasma loss rate. If these effective $\dot{M}$ quantities are not balanced, then the magnetodisc will grow/shrink until momentum balance is once again achieved. In this sense, the neutral-plasma interaction is fundamental to understanding the MI coupling within the magnetosphere, as well as the coupling of the magnetosphere with the solar wind.

\subsection{Summary}

The interaction between satellite-generated neutral gases and the magnetospheric plasma environment is critical for the formation of giant planet magnetodiscs. In the absence of a source of energy to heat the electrons, ionization of the neutral clouds would not occur. Yet the ionization of the neutral gas leads to a flow of momentum and energy from the planet that in turn heats the plasma. This feedback process can reach a non-linear tipping point where the plasma environment can rapidly erode the neutral clouds. At Saturn, the inner magnetosphere is dominated by neutral gas as electron temperatures near Enceladus are not sufficient to tip the balance in favour of plasma. But at Jupiter, the electron temperatures are sufficient to yield a plasma-dominated inner magnetosphere. The net result is that Saturn's magnetosphere is only slightly inflated by the internal plasma source when compared to Jupiter's highly inflated magnetosphere (Fig. 8). It follows then that Jupiter's magnetodisc is dominated by plasma pressure $(\beta>1)$ while the plasma pressure in Saturn's magnetodisc is comparable to the magnetic field pressure (i.e. $\beta \sim 1$ ). These very different conditions can significantly affect the solar wind interaction with these magnetospheres. 
(a) Small-Scale Injecttions and Energization $(L<9)$
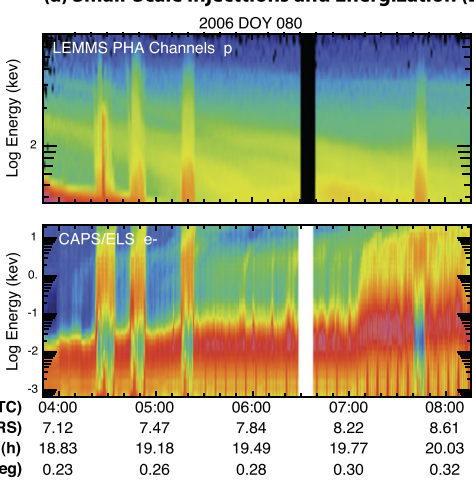

(b) Large-Scale Injections and Energization ( $L \geq 9)$ 2004 DOY 184
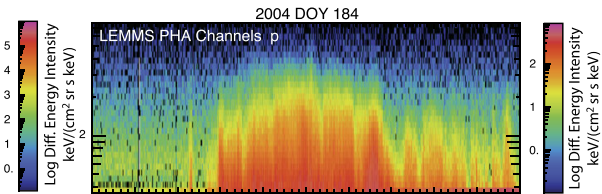
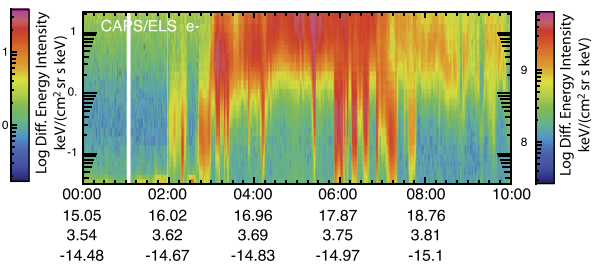

Fig. 9 In-situ observations of small- (left) and large-scale (right) injections at Saturn obtained by Cassini/MIMI (magnetospheric imaging instrument). The upper panel shows protons measured by LEMMS (low energy magnetospheric measurement system) and the lower panel shows electrons measured by CAPS (Cassini plasma spectrometer). The small-scale injections are observed predominantly inside $9 \mathrm{R}_{\mathrm{S}}$ and have a very narrow spatial extent $\left(\ll 1 R_{S}\right)$. The large-scale injections are observed predominantly beyond $9 R_{S}$ and have a relatively broad spatial extent (several $\mathrm{R}_{\mathrm{S}}$ ). See Fig. 10 for an example of the instantaneous global distribution of large-scale injections

\section{Injections and Their Role in Magnetodisc Formation and Stability}

\subsection{In-Situ Picture}

The occurrence of energetic particles, up to several hundreds of $\mathrm{keV}$, is a particularly apparent feature of the Saturnian magnetosphere. In the Jovian magnetosphere, such intensifications of energetic particles are less apparent and occur on top of a "sea" of already enhanced ion intensities, which is most likely due to the lack of a significant radial gradient in phasespace density (Mauk et al. 1999). In Saturn's magnetosphere, the injection of energetic particles seems to stem from two main fundamental mechanisms: (1) small-scale injections observed roughly inside of $9 \mathrm{R}_{\mathrm{S}}$ associated with centrifugally driven interchange (Kennelly et al. 2013; Rymer et al. 2009), and (2) large-scale injections observed roughly beyond $9 \mathrm{R}_{\mathrm{S}}$, likely associated with planetward propagation due to magnetic field line tension (or buoyancy) set up by reconnection processes in the magnetotail region (Pontius and Hill 1989; Bunce et al. 2005; Mitchell et al. 2009, 2015). Both of these mechanisms are fundamental to the energization and transport of plasma and therefore comprise an important aspect of magnetodisc formation and stability.

Figure 9 illustrates both types of injection. The left-hand column shows the small-scale injections that are associated with $\ll 1 \mathrm{R}_{\mathrm{S}}$ particle intensifications. These have been associated with centrifugally driven interchange-type instabilities. The right-hand column provides an example of a large-scale injection observed post-midnight, around about $17 \mathrm{R}_{\mathrm{S}}$. Its lack of energy dispersion indicates that this is a rather young injection. The injected particle distributions will disperse as they drift due to the sub-corotational electric field (i.e. the convective electric field due to bulk rotation), and magnetic gradient/curvature forces. Such particle dispersions have been observed both in situ (Mauk et al. 2005) and in Energetic Neutral Atom (ENA) images (Brandt et al. 2008) obtained by Cassini INCA (Ion Neutral Camera). An initial ion population, evenly distributed (spatially) over finite intervals in radial distance and azimuth, will therefore disperse and form a spiral pattern, with the higher 


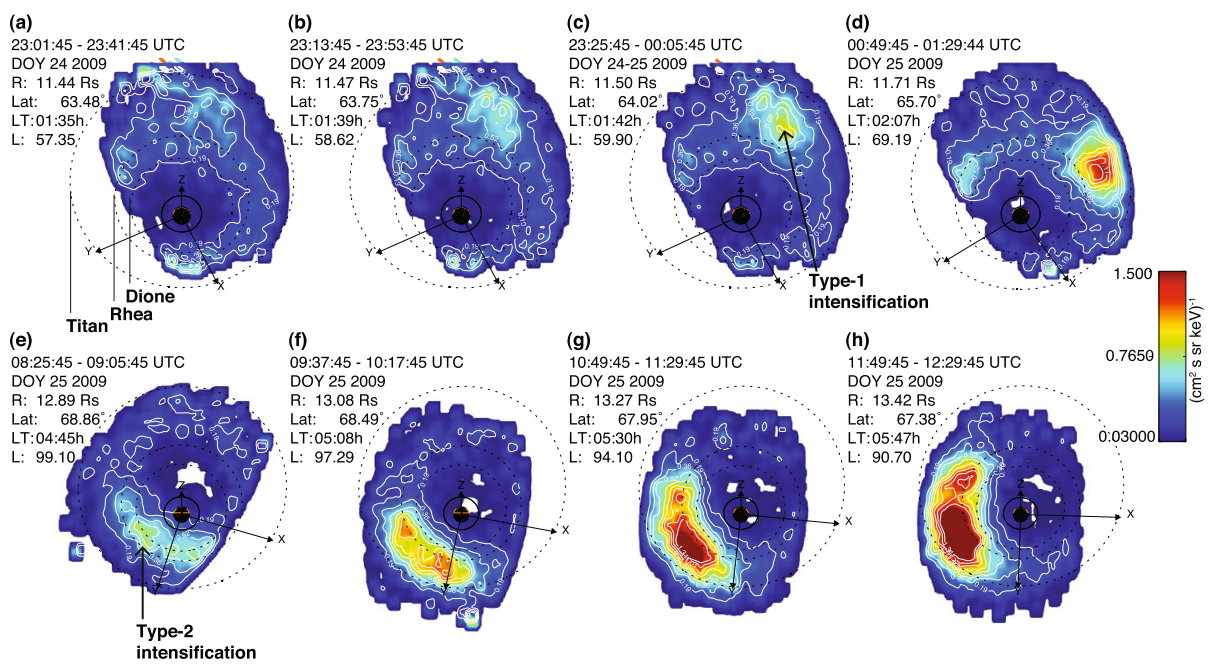

Fig. 10 Hydrogen ENA image sequence obtained from the southern hemisphere by Cassini/INCA in the 24-55 keV range. The sequence shows the first type of large-scale injection with an inward radial propagation from the tail. The injected population then drifts around Saturn due to the sub-corotational electric field, gradient and curvature forces. The drift trajectory is slightly radially inward. As the population reaches the pre-midnight sector it re-intensifies due to yet unknown processes. Position information for the spacecraft is reported in each panel. The axes shown correspond to the SZS coordinate system, in which the Z axis is parallel to the planet's rotational axis and the planet-Sun direction lies in the XZ plane

energy ions drifting faster than those of lower energy. Although this behaviour has been observed several times, there are other behaviours that remain unexplained.

It is important to remember that the term 'interchange instability' represents a broad range of magnetospheric phenomena and is not exclusively reserved for the small-scale injections, that are most likely centrifugally driven. Large-scale injections can plausibly also be the signature of an interchange-type instability, but they are more likely to be driven by the relatively strong buoyancy (with respect to the ambient plasma) of hot, low-density flux tubes which are generated through magnetic reconnection processes in the magnetotail.

\subsection{Global Picture}

The relation between the small- and large-scale injections is best observed in remote global images of the energetic ion distributions obtained by INCA. This instrument uses a technique called Energetic Neutral Atom (ENA) imaging, which is based on detecting the direction, energy and species of ENAs produced through charge exchange between singly charged energetic ions and neutral species.

As illustrated in Figs. 10 and 11, there are two main categories of ENA intensifications observed by INCA. The first type ('Type 1') occurs as an inward and slightly azimuthally propagating intensification, and is visible from about $20 \mathrm{R}_{\mathrm{S}}$ in the midnight/post-midnight sector. The second type ('Type 2') is re-brightening of an already existing emission region in the pre-midnight/midnight region. We describe the observations in more detail below.

The hydrogen ENA image sequence shown in Fig. 10 was obtained from the Southern hemisphere on DOY 24-25 2009 in the 24-55 keV energy range. The apparent hard edges of the image are the limitations of the FOV (field of view) of INCA. Figures 10a-10d show 


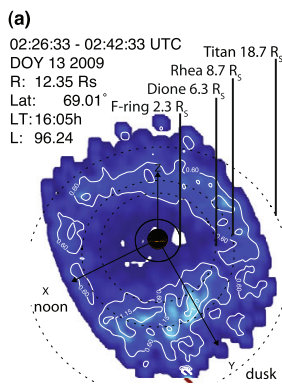

(b)

03:02:33 - 03:22:33 UTC

DOY 132009

R: $12.26 \mathrm{Rs}$

Lat: 69.03

LT: $16: 18 \mathrm{~h}$

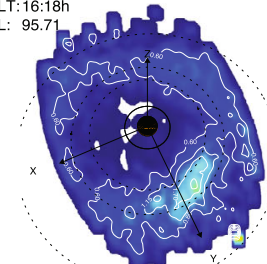

(e)
05:02:33 - 05:22:33 UTC

DOY 132009

R: $11.95 \mathrm{Rs}$

Lat: $\quad 68.61^{\circ}$

LT: 17:04h

L: 89.81

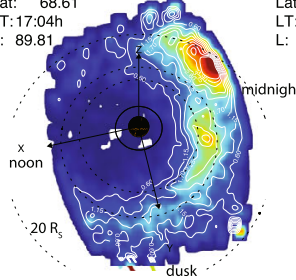

(f)

05:42:34 - 06:02:34 UTC

DOY 132009

R: $11.84 \mathrm{Rs}$
Lat: $\quad 67.29$

Lat: $\quad 67.29$

LT:17:19h

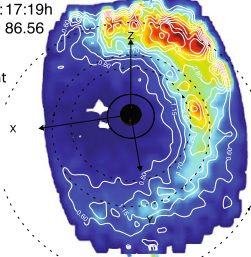

(c)

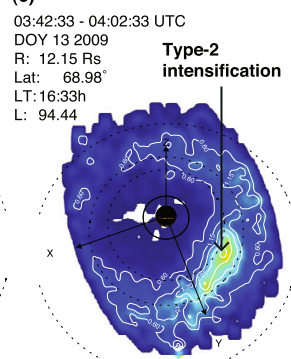

(g)

06:22:34 - 06:42:34 UTC DOY 132009 R: $11.74 \mathrm{Rs}$ Lat: $\quad 67.88^{\circ}$ LT: $17: 34$ 82.8

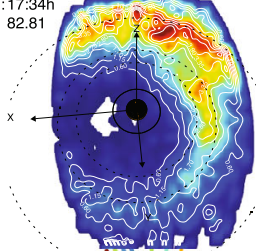

(d)

04:22:33 - 04:42:33 UTC DOY 132009 Type-1 $\mathrm{R}: 12.05 \mathrm{Rs}$ intensification Lat: $68.84^{\circ}$
LT: $16: 49 \mathrm{~h}$ L: 92.46

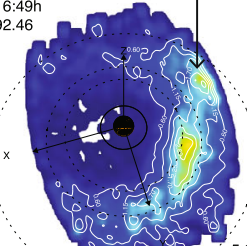

(h)

07:02:34 - 07:22:34 UTC DOY 132009 R: $11.64 \mathrm{Rs}$ Lat: $67.38^{\circ}$ LT: $17: 49 \mathrm{~h}$ : 78.67

Fig. 11 Hydrogen ENA image sequence obtained from the northern hemisphere by Cassini/INCA in the 24-55 keV range. The sequence displays the occurrence of a Type-2 intensification together with a Type- 1 . Although tempting to suggest, it is not yet clear if Type-2 intensifications trigger Type-1

a Type 1 intensification becoming first visible around $20 \mathrm{R}_{\mathrm{S}}$ at the upper edge of the FOV, and then propagating inward to the post-midnight sector and intensifying. The intensification could be due to two things: (1) energization of the ions leading to higher ion differential intensities, and/or (2) propagation in to regions of higher neutral densities leading to higher ENA differential intensities. Although this Type 1 intensification shows intriguing fine structure, it is not clear if it is real or is an effect of counting errors and running averages used for the images. Neither can be excluded at this stage.

Figure 10e shows how the Type 1 injection has now drifted around to the dusk sector. There is a decrease of ENA intensities from Figs. 10d to 10e, which is attributed to charge-exchange loss. However, in subsequent images of Figs. 10f to 10h, the intensities are increasing. This kind of (re)intensification is referred to as Type 2. Fine structure is coherent through the observations of the Type 2 intensification and is arguably real, since the image averages are well separated in time, but still display the same general fine structures.

Figure 11 illustrates how a Type 1 intensification can sometimes be preceded by a Type 2 intensification. The sequence shows ENA images in the 24-55 keV range, obtained from the northern hemisphere. Again, a Type 2 intensification of a previous, dimmer population occurs in the pre-midnight sector as shown in Figs. 11a-11c. However, as the Type 2 intensification reaches midnight in Fig. 11d, a Type 1 event is visible at the upper right edge of the FOV of INCA, and propagates rapidly in azimuth (at radial distance about $20 \mathrm{R}_{\mathrm{S}}$ ), at about twice the rate of rigid planetary corotation-which is consistent with the modeling results by Jia et al. (2012). The reader should be cautioned here that the fine structure of the Type 1 intensification seen in Figs. 11e-11h is likely to be an artefact of the collimator blades of the INCA camera or counting noise.

The Type 1 intensification is most likely the global manifestation of the large-scale injections presented in Fig. 9. This statement is supported by the location and size of the 
non-dispersive, large-scale injection in Fig. 9. Perhaps, more importantly, both Type 1 intensifications observed by INCA and the large-scale injections observed in-situ appear to be associated with low-frequency extensions (LFE) of the Saturn Kilometric Radiation (Lamy et al. 2013; Mitchell et al. 2015). In addition, modelling by Jia et al. (2012) shows fast plasma flows returning to the inner, post-midnight magnetosphere following reconnection processes in the tail. Therefore, the preliminary conclusion that we draw is that reconnection in the magnetotail creates planetward, fast flows that are driven by the buoyancy of the associated low-density plasma (Pontius and Hill 1989). As the fast plasma flow reaches the inner magnetosphere it leads to plasma energization, either through inward radial transport to regions of higher magnetic field strength, and/or through particle interaction with the thin dipolarization fronts of the plasma flow burst. This type of process is very similar to that believed to be causing the main ion energization in the terrestrial magnetosphere (Runov et al. 2011).

The cause of the Type 2 intensifications is still under investigation, but possible explanations include small-scale plasma injections, based on the radial range where these are observed in-situ. The fact that the Type 1 intensification drifts radially inward to then transform into a Type 2 intensification also supports the idea that small-scale injections are playing a role in transporting plasma radially inward-thus leading to enhanced ENA intensity due to ion energization and the higher neutral densities encountered. Although radial, inward motion is not apparent in the INCA images presented here, Carbary et al. (2008b) demonstrated that a slight tendency for inward propagation in the INCA image sequences was statistically significant. Thomsen et al. (2012) provides further evidence from a range of in-situ measurements that this is indeed a feature of the drifting ion populations. A potentially important clue may also come from the fact that Type- 2 intensifications occur more frequently at periods close to that of Saturn's rotation than do the Type-1 intensifications. Brandt et al. (2012) reported four consecutive occurrences of Type-2 ENA intensifications close to the orbit of Dione with a Type-1 intensification only after the fourth Type- 2 intensification. As an independent confirmation of the regular nature of the Type-2, Mitchell et al. (2015) reported a close relation between the Type-2 ENA intensifications and narrow-band emissions, although the exact relation between the two remains unclear.

\section{The Interchange Instability}

\subsection{The Interchange Instability Criterion}

The structure and dynamics of giant planet magnetospheres are dominated by the presence, within the magnetospheric cavity, of several plasma sources and by the fast planetary rotation. The various plasma populations are trapped by the planetary magnetic field and the relatively cold plasma is confined near the equatorial plane by the action of centrifugal force, thereby giving rise to a thin disk of (sub)corotating plasma. The redistribution of the locally created plasma throughout the magnetospheric system is one of the fundamental dynamical processes occurring in giant planet magnetospheres. This redistribution is achieved through plasma transport perpendicular to magnetic field lines (see Sect. 1). The exact mechanisms responsible for this transport are not yet completely understood, with the transport probably operating through different modes and on different scales, both spatial and temporal. It is, however, widely believed that the outward plasma transport is triggered by the centrifugal instability, a Rayleigh-Taylor type instability in which the centrifugal force plays the role of the gravitational force, and that it proceeds through 
the interchange of magnetic flux tubes (e.g., Melrose 1967; Ioaniddis and Brice 1971; Hill 1976). Physically, under the action of the centrifugal and magnetic buoyancy forces, mass-loaded flux tubes tend to exchange positions with relatively empty flux tubes, located further out in the magnetosphere.

In terms of in situ observations, there is no direct evidence of outward-moving flux tubes laden with cold plasma. However, the outflow velocity in Jupiter's magnetodisc has been inferred by Russell et al. (2000) through the use of various techniques. One of these involved the use of magnetodisc field measurements and stress-balance arguments to estimate a plasma mass density, and then equating the total rate of radial mass outflow to the rate at which plasma mass is added by the Io source. At a distance of $25 \mathrm{R}_{\mathrm{J}}$, for example, the estimated velocity is $29 \mathrm{~km} \mathrm{~s}^{-1}$. Considering the density profile of plasma nearer the Io torus (6-9 R ), Russell et al. (2000) estimated smaller outward radial velocities $\sim 9-68 \mathrm{~m} \mathrm{~s}^{-1}$ in this region. These authors also pointed out that magnetic signatures of empty flux tubes in the Io torus region comprised a small fraction, $(\sim 0.4 \%)$, of the total time covered by the relevant data. This aspect supports the picture of empty flux tubes which carry magnetic flux inwards at relatively high speeds, in order to compensate for the outward transport of flux via the motion of a body of cold plasma which occupies the majority of the magnetodisc volume (Russell et al. 2000 estimate that the inward-moving tubes have speeds $\sim 250$ times greater than the outward-moving ones; see also Sect. 1). In this picture, the net radial flux of plasma mass would be outward-directed.

Interchange motions have been extensively discussed in the literature under a variety of simplifying assumptions, sometimes unrealistic and unproven. A review of this abundant literature can be found in the introductory section of Ferrière et al. (1999). Gold (1959) was the first to introduce the concept of interchange of magnetic flux tubes in the magnetospheric context (see also Sect. 1). His so-called strict interchange model assumes a one-to-one interchange between magnetic flux tubes enclosing the same amount of magnetic flux and thus leaving the shape of the field lines unchanged, and the total magnetic energy of the system unperturbed. Cheng (1985) pointed out much later that this model is at odds with the requirement of total pressure balance, and that a realistic flux tube interchange must be accompanied by a corresponding change in field magnitude. The so-called generalized interchange model of Southwood and Kivelson (1987) still assumes that the interchanging flux tubes preserve everywhere the direction of the local magnetic field, but they relax the condition that the energy density of the magnetic field is unperturbed by the interchange. Both models involve approximations for actual interchange motions of plasma elements, which generally entail distortions of the direction of magnetic field lines, preserving the equilibrium of total pressure (plasma plus magnetic pressure).

Newcomb (1961) studied the influence of the gravitational field and of stratification on the three modes of ideal magnetohydrodynamics (the Alfvén mode, the fast and slow modes). He considered a plasma confined in a horizontal magnetic field distribution by a uniform, vertical gravitational field. Newcomb (1961) identified two convective wave modes for this system, whose dispersion relation is influenced by the stratification of the plasma in the vertical direction (for the case of a wave vector nearly perpendicular to the magnetic field). These two modes can be distinguished by their behaviour in the limit of zero parallel wave vector (with respect to the magnetic field). In this limit, the convective wave modes occur as two types, and were given the name of quasi-interchange modes: one of these modes gives rise to plasma motions mainly across the magnetic field lines (hereafter denoted the interchange or type 1 mode), while the other two modes give rise to plasma motions mainly along the magnetic field lines (hereinafter denoted as translation or type 2 modes). These quasi-interchange modes have been studied more comprehensively than in 
previous attempts by Ferrière et al. (1999) for the case of collisional plasmas, and by Ferrière and André (2003) for the case of collisionless proton-electron plasmas. These studies take into account the effect of both gravitation and magnetic field line curvature. André and Ferrière (2004) derived the local stability criterion of small-scale, low-frequency modes in stratified, rotating plasmas, consisting of multiple ion species and characterised by gyrotropic, bi-Maxwellian distribution functions. They obtained a criterion that consists of the simple superposition of two distinct criteria; the first being related to instabilities triggered by thermal pressure anisotropies, and the second corresponding to instabilities triggered by stratification. All low-frequency modes (Alfvén, fast, slow and mirror modes) were found to be stable over all wave vectors, if and only if:

$$
\mathcal{F} \geq 0 \quad \text { and } \quad \mathcal{M} \geq 0 \quad \text { and } \quad \varpi_{0}^{2}-\frac{g_{1}^{2}}{\mathcal{M}} \geq 0 .
$$

The restricted number of parameters appearing in the stability condition (8) are defined as follows. Firstly, the parameter $\mathcal{F}$ is given by

$$
\mathcal{F}=V_{A}^{2}+C_{\perp}^{2}-C_{\|}^{2},
$$

and thus involves the Alfvén speed, $V_{A}$ and the sound speeds in directions perpendicular and parallel to the magnetic field (respectively $C_{\perp}$ and $C_{\|}$). The parameter $\mathcal{M}$ is defined as:

$$
\mathcal{M}=V_{A}^{2}+\gamma_{\perp_{\perp}} C_{\perp}^{2}-\frac{\gamma_{\perp_{\|}}^{2} C_{\perp}^{4}}{\gamma_{\|_{\|}} C_{\|}^{2}},
$$

where the $\gamma$ coefficients, due to limitations of space, are not given here in their full forms, but may be found in equations (B9)-(B12) of André and Ferrière (2004). These coefficients are complicated functions that contain the relevant information on the composition and velocity distribution of the collisionless plasmas considered. $\mathcal{F}$ and $\mathcal{M}$ have physical meaning, and are respectively linked to the firehose and mirror instability criteria.

The definition of the final parameter in (8) is

$$
\begin{aligned}
\varpi_{0}^{2}-\frac{g_{1}^{2}}{\mathcal{M}}= & \left(\frac{\nabla \rho}{\rho}-\frac{\mathbf{g}}{\gamma_{\|\|} C_{\|}^{2}}\right) \cdot \mathbf{g} \\
& +\left[\frac{\nabla\left(2 P_{\mathrm{M}}+P_{\perp}-P_{\|}\right)}{\rho}-\mathcal{F} \mathbf{c}\right] \cdot \mathbf{c} \\
& -\frac{\left(\left[\left(1-\frac{\gamma_{\perp_{\|}}}{\gamma_{\|\|}} \frac{C_{\perp}^{2}}{C_{\|}^{2}}\right) \mathbf{g}+\mathcal{F} \mathbf{c}\right] \cdot \widehat{e}_{g}\right)^{2}}{\mathcal{M}} .
\end{aligned}
$$

This is a threshold parameter with the dimension of frequency squared. In these definitions, $\rho$ denotes the plasma mass density, $\mathbf{g}$ the effective gravity (including a centrifugal component) with unit vector $\widehat{e}_{g}=\mathbf{g} / g$, c the magnetic curvature vector, $C_{\perp}^{2}=\left(P_{\perp} / \rho\right)$, $C_{\|}^{2}=\left(P_{\|} / \rho\right)$ where the corresponding plasma pressures are $P_{\perp}$ (perpendicular to the magnetic field) and $P_{\|}$(parallel to the magnetic field). $P_{\mathrm{M}}$ denotes magnetic pressure. All the physical quantities and parameters appearing in these expressions pertain to the equilibrium state, where they are all considered to be invariant along magnetic field lines, with the additional assumption that the effective gravity and magnetic curvature vectors are parallel.

The first two inequalities in (8) are required to guarantee stability against the anisotropydriven firehose and mirror instabilities, respectively. Together, they constitute the necessary and sufficient condition for the stability of low-frequency modes in a uniform medium. The last inequality represents the effect of stratification in the plasma, and is required to 
prevent the growth of stratification-driven instabilities. Stratification and rotation alter the physical characteristics of the Alfvén, slow and mirror modes in the limit of nearly fieldperpendicular wave vectors, as discussed previously. The types of unstable modes in the different domains of parameter space depend on the four parameters appearing in (8) and on the sign of the generalized Rayleigh-Taylor frequency $\omega_{0}$, given by:

$$
\begin{aligned}
\omega_{0}^{2}= & \left(\frac{\nabla \rho}{\rho}-2 \mathbf{c}\right) \cdot \mathbf{g} \\
& +\left[\frac{\nabla\left(2 P_{\mathrm{M}}+P_{\perp}-P_{\|}\right)}{\rho}-\left(\mathcal{F}-3 C_{\|}^{2}\right) \mathbf{c}\right] \cdot \mathbf{c} \\
& -\frac{\left[\mathbf{g}+\left(V_{\mathrm{A}}^{2}-C_{\|}^{2}\right) \mathbf{c}\right]^{2}}{V_{A}^{2}+2 C_{\perp}^{2}} .
\end{aligned}
$$

Interestingly, $\omega_{0}^{2}$ depends only on fluid parameters and not on the exact composition of the multispecies plasma, whereas the details of the plasma composition enter the overall stability criteria equation (8), such that composition plays a role in determining the plasma's stability against all quasi-interchange modes.

The rest of this section is devoted to a discussion of the stability criterion of the interchange mode of type 1 (quasi-interchange mode) in giant planet magnetospheres. In these environments $\mathcal{F} \geq 0$, which implies that the condition $\omega_{0}^{2} \geq 0$ is necessary and sufficient for the stability of the interchange mode.

\subsubsection{Case Including Effective Gravity (Centrifugal Force) Only}

If the equilibrium magnetic field is assumed to be straight (i.e. no field line curvature), then the interchange instability criterion reduces to the following:

$$
\mathbf{g} \cdot\left(\frac{\nabla \rho_{0}}{\rho_{0}}-\frac{\mathbf{g}}{V_{A}^{2}+2 C_{\perp}^{2}}\right) \geq 0 .
$$

Although the plasma pressure gradient does not influence the interchange instability criterion, the thermal pressure itself has a stabilizing effect, since it enters the stability criterion through the perpendicular sound speed. In the cold plasma approximation, $C_{\perp}^{2}=0$ and the stability criterion reads (after integration along magnetic field lines):

$$
\mathbf{g} \cdot \nabla \eta \geq 0
$$

where $\eta=\int(\rho / B) d s$ is the mass per unit magnetic flux, and one recovers the classical result of the strict interchange model of Gold (1959). This criterion is frequently used in situations where the plasma beta is less than unity. This is typically the case in the innermost magnetospheres of Jupiter and Saturn.

\subsubsection{Case Including Curvature Force Only}

In the magnetospheric context for rapidly rotating systems, the magnetic curvature force often dominates over that of gravitation (Southwood and Kivelson 1987). In the case of isotropic collisional plasmas, the stability criterion for the interchange mode can be rewritten in terms of the volume of a magnetic flux tube $V_{0}$ and the radial gradient of the thermodynamic adiabatic invariant $P_{0} V_{0}^{\gamma}$, and reads:

$$
-\mathbf{c} \cdot \nabla\left(P_{0} V_{0}^{\gamma}\right) \geq 0
$$


in which the vector $\mathbf{c}$ is directed planetward. This expression thus coincides with the interchange stability condition obtained by Southwood and Kivelson (1987). A collisional plasma would therefore be stable against interchange motions provided its thermal pressure decreases outward less rapidly than for the case of adiabatic transport. For completeness, André and Ferrière (2004) have shown that a gyrotropic, collisionless plasma with isotropic thermal pressure at equilibrium is always more stable than the corresponding isotropic, collisional plasma.

\subsubsection{Competing Effects of Cold and Hot Plasma Populations}

We now turn our attention to the question of whether the local stability criterion against the interchange mode is fulfilled in the Io torus at Jupiter, and in the inner magnetosphere of Saturn. Following the Voyager encounters of Jupiter (specifically observations of the Io torus), it has been proposed that the hot/energetic plasma population plays a critical role in controlling radial plasma transport. For example, Siscoe et al. (1981) suggested that the thermal pressure gradient of the hot plasma in the Io torus could reduce or enhance transport via interchange, depending on whether it is directed in the same or opposite sense to the effective gravity (impoundment mechanism). Mauk et al. $(1996,1998)$ tested this suggestion by calculating the radial gradient of $P_{0} V_{0}^{\gamma}$ based on the hot/energetic plasma pressures inferred from the Voyager and Galileo observations. They concluded that the hot plasma distribution indeed impeded radial transport in both cases, and that this impoundment by the hot plasma had probably been more efficient during the Voyager era than during the Galileo era, when the hot plasma appeared significantly depleted (Fig. 12a).

Chen (2003) numerically studied the motion of a mass-loaded magnetic flux tube within empirical models of the Io torus at equilibrium, using a MHD approach. André and Ferrière (2004) also used different equilibrium models of the Io torus in which low- and high-energy plasma components have a specified radial distribution at the Jovian centrifugal equator, similar to the approach of Chen (2003), in order to test the interchange stability criterion. Both studies showed that the relative flux tube content of cold to hot plasma plays an important role in plasma transport for the Jovian system. These studies also showed that most of the torus is close to marginal stability against interchange. This is expected, since the injection of plasma at Io's orbit initially gives rise to unstable density gradients, which trigger plasma transport, which in turn acts to reduce the unstable gradients and restore a configuration closer to marginal stability. In both studies, the Io torus appeared stable against interchange at the time of Voyager when both the contributions of cold and hot plasma were taken into account, in agreement with the conclusions of Mauk et al. (1998). Unstable regions were identified for the Galileo period, in close correlation with the regions where clear observational signatures of the instability were reported (e.g., Kivelson et al. 1997a).

Whereas similar detailed studies of interchange stability remain to be done in the case of Saturn (although see Sect. 5), a few preliminary conclusions may be drawn. Similar to the proposed impoundment mechanism at Jupiter, a large fraction of the inner magnetosphere of Saturn is a 'battleground' between the competing influences of the interchange instability of the cold, internally generated plasma, and the interchange stability of the hotter, energized plasma (Mauk et al. 2009). At Saturn, Sittler et al. (2008) have shown that the main plasma source is at $L=6$ because that is where the ambient electrons become hot enough to produce fast collisional ionization (Fig. 12b). Outside of the radial location of this peak plasma source at $L=6$, Saturn's magnetosphere should therefore be unstable to centrifugal interchange in the cold plasma limit —and this picture is 

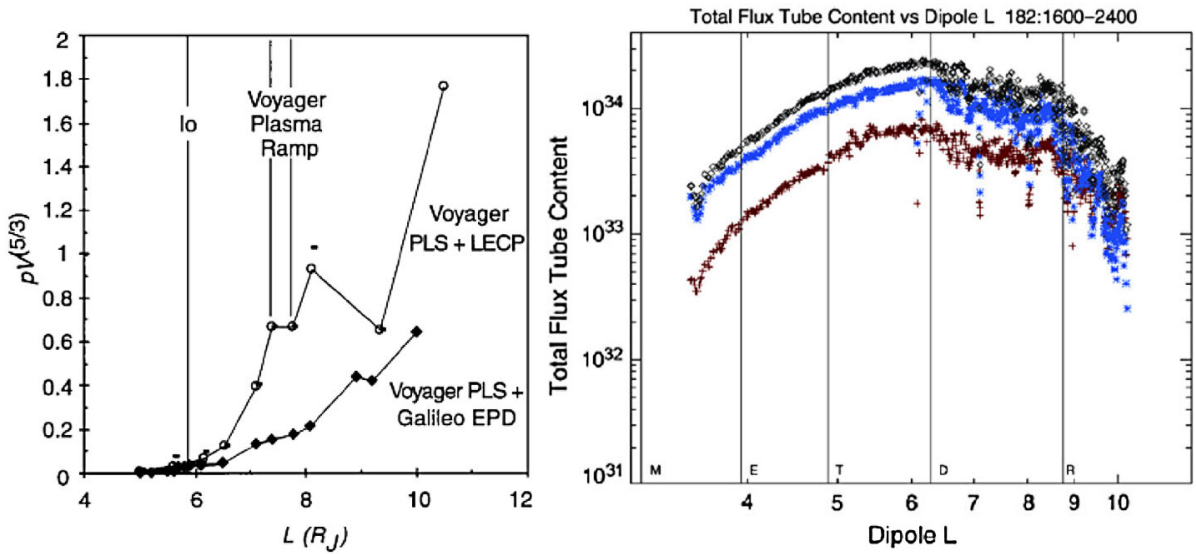

Fig. 12 (a) Radial profile of the thermodynamic invariant for combined Voyager PLS, Voyager LECP and Galileo EPD data obtained at Jupiter. Figure taken from Mauk et al. (1998); (b) total flux tube content for water-group ions (in blue), protons (in red) and total ions (in black) versus dipole $L$, from Cassini CAPS observations at Saturn. Figure reprinted from Sittler et al. (2008), Copyright (2007), with permission from Elsevier

indeed in close agreement with Cassini observations of plasma injection events beyond that distance. This simple picture should, however, be contrasted with the analysis of Sergis et al. (2007), which showed that most of the plasma pressure is carried by ions in the energy range $10-150 \mathrm{keV}$, and that the hot plasma pressure increases radially outward to acquire peak values around $L=10$. The observational signatures of centrifugal interchange are found to be clustered between $5-6 R_{S}$ and $10-11 R_{S}$, exactly where one observes the respective peak values in both cold plasma flux tube content and hot plasma pressure.

\subsubsection{Coriolis Influence on the Interchange Instability}

The influence of planetary rotation is felt through the centrifugal and Coriolis forces in a rotating frame of reference. Whereas the centrifugal force is included in most studies of the interchange instability, the Coriolis force and its impact are generally not taken into account. Vasyliūnas and Pontius (2007) have recently shown that the presence of the Coriolis force renders the growth rate of the instability complex, adding an imaginary component to this rate. This is equivalent to a real oscillation frequency, with the consequence of making the pattern of fluctuations rotate azimuthally, relative to the corotating frame of reference. The Coriolis force also acts to reduce the magnitude of the real growth rate. The growth rate depends on the ratio of azimuthal to radial wavelength and acquires maximal values for structures elongated in the radial direction. All the Coriolis effects become, however, negligible in the limit of very short azimuthal wavelengths, in agreement with the results of André and Ferrière (2007) that are restricted to that specific case. Although not a generally valid result, neglect of the Coriolis force effects is a reasonable approximation, as long as the fastest-growing instabilities are those with very short azimuthal wavelengths. This result may therefore hold quite well, in general, for practical applications to the magnetospheres of Jupiter and Saturn, although a few additional caveats were mentioned by Vasyliūnas and Pontius (2007). 

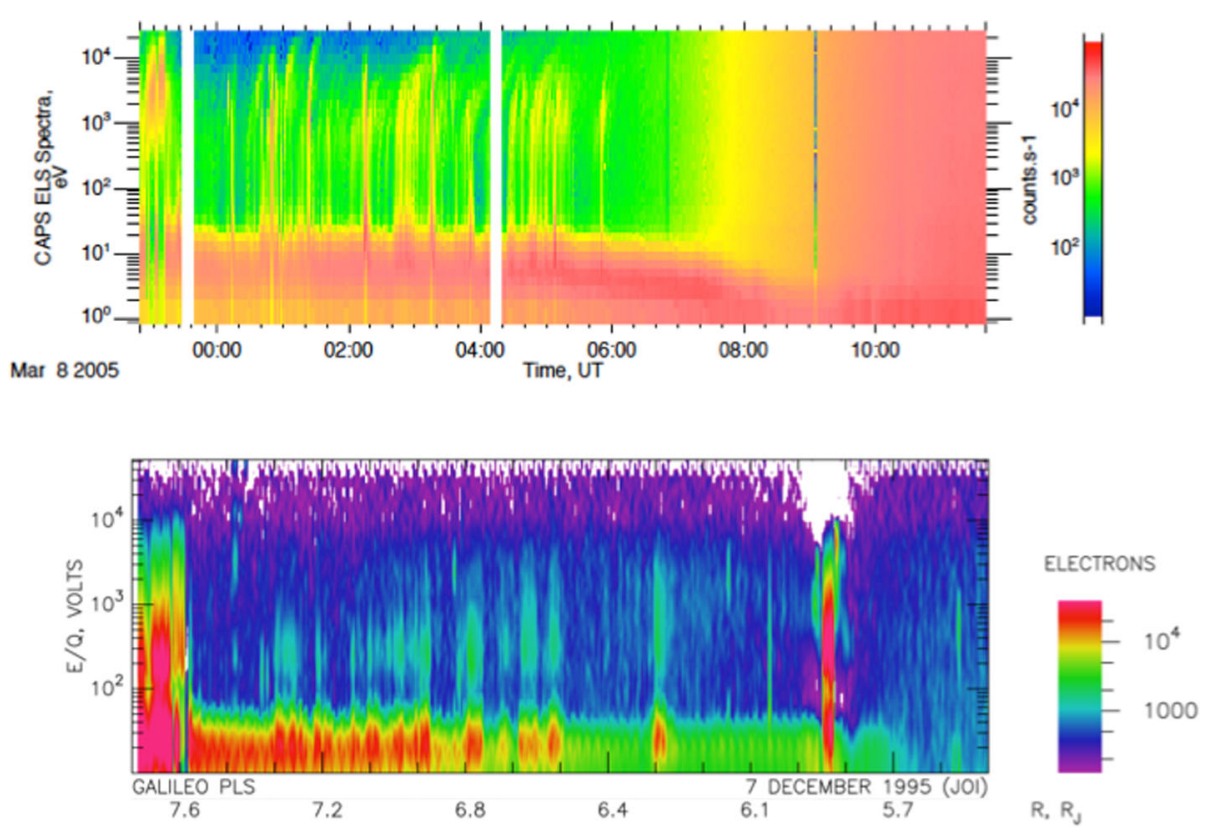

Fig. 13 Energy-time spectrogram of electrons at Saturn (top, Cassini CAPS; figure made with the AMDA tool available at CDPP, http://cdpp.cesr.fr) and Jupiter (bottom, Galileo PLS; figure taken from Frank and Paterson 2000)

\subsection{In Situ Signatures of the Interchange Process}

The recent Galileo and Cassini space missions have amply documented the Jovian and Saturnian magnetospheric systems. In particular, these missions have provided us with new observations and new insights into the mechanisms responsible for the outward plasma transport. There is now considerable observational evidence that centrifugally driven flux tube interchange is at play in the corotation-dominated regions of the Jovian and Saturnian magnetospheres, and that this process contributes to the redistribution of plasma throughout these systems. Signatures of intermittent, short-lived, mass-loaded and relatively empty flux tubes in the Io torus have been detected by the Galileo spacecraft, in orbit around Jupiter from December of 1995 to September of 2003 (e.g. Kivelson et al. 1997a; Thorne et al. 1997). Similar signatures have been observed in the E-ring of Saturn by the Cassini spacecraft, in orbit around Saturn since July 2004 (André et al. 2005; Burch et al. 2005, 2007; Hill et al. 2005; Leisner et al. 2005; Mauk et al. 2005; Rymer et al. 2008) and have lent further support to the notion that the centrifugal instability lies at the root of the outward transport.

In both magnetospheres, the reported plasma signatures consisted of discrete hot plasma injection events accompanied by longitudinal dispersion arising from magnetic gradient and curvature drift (Figs. 13, 14). The magnetic signatures of the injections consist of shortduration, sharp-bounded intervals of either enhanced or depressed magnetic pressure, and whose plasma content differs significantly from that of the surrounding medium (Fig. 14). These signatures were consistent with expectations for the interchange of magnetic flux tubes containing dense and cold plasma with those containing tenuous and hot plasma that originate farther out in the magnetosphere. Compared to Jupiter, Saturn's magnetosphere provides a somewhat better 'laboratory' for understanding the observational properties of 

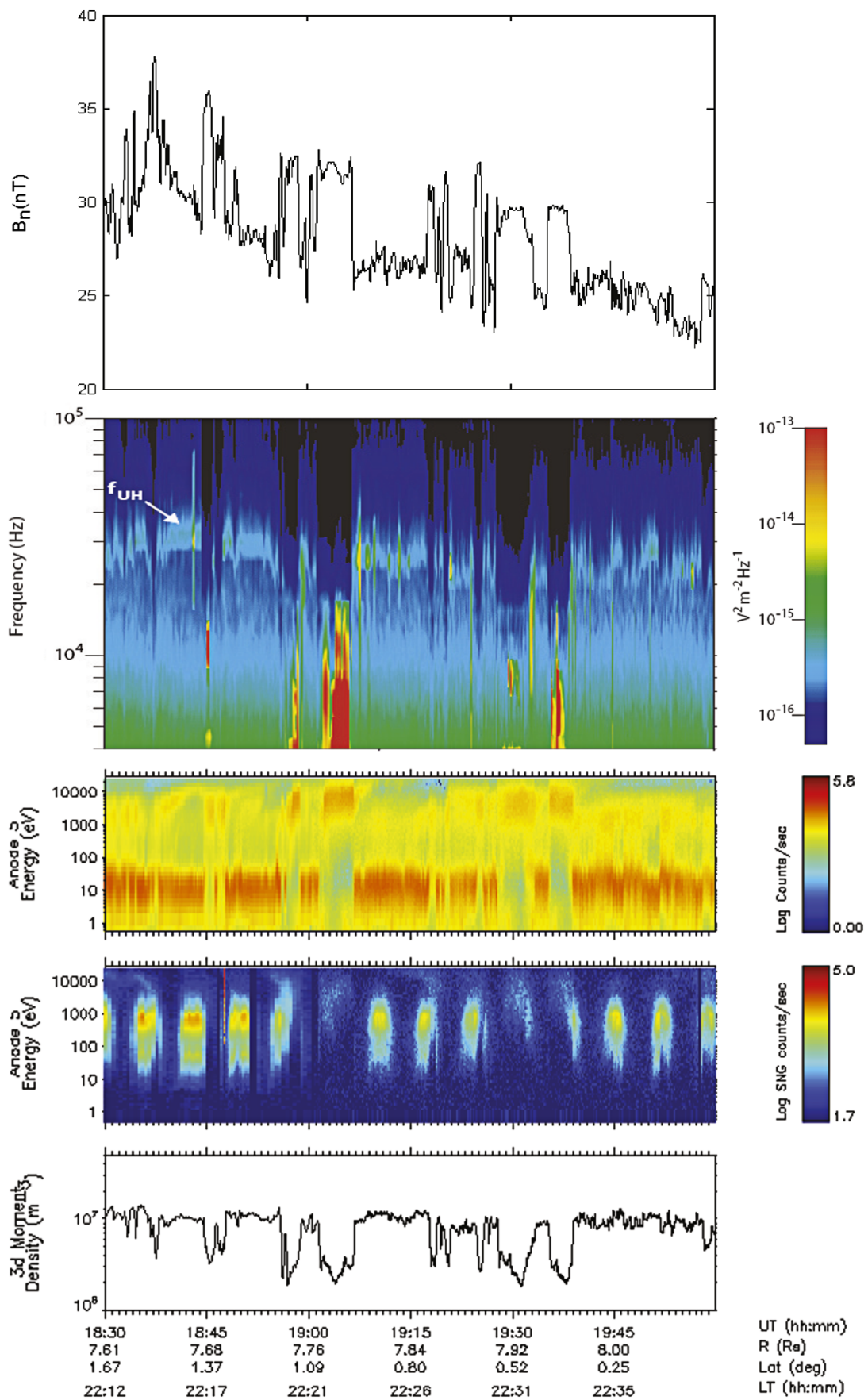

Fig. 14 Multi-instrument analysis of a series of injection events on October 28, 2004, showing correlations between (from top to bottom panels) enhancements in the magnetic field, the disappearance of the upper hybrid emission band, the depletion of the low-energy plasma population, the coincident appearance of a hot plasma component, and significant density drop-outs. These events occur inside the well-defined boundaries of the interchanging magnetic flux tubes. Figure adapted from André et al. (2007) 


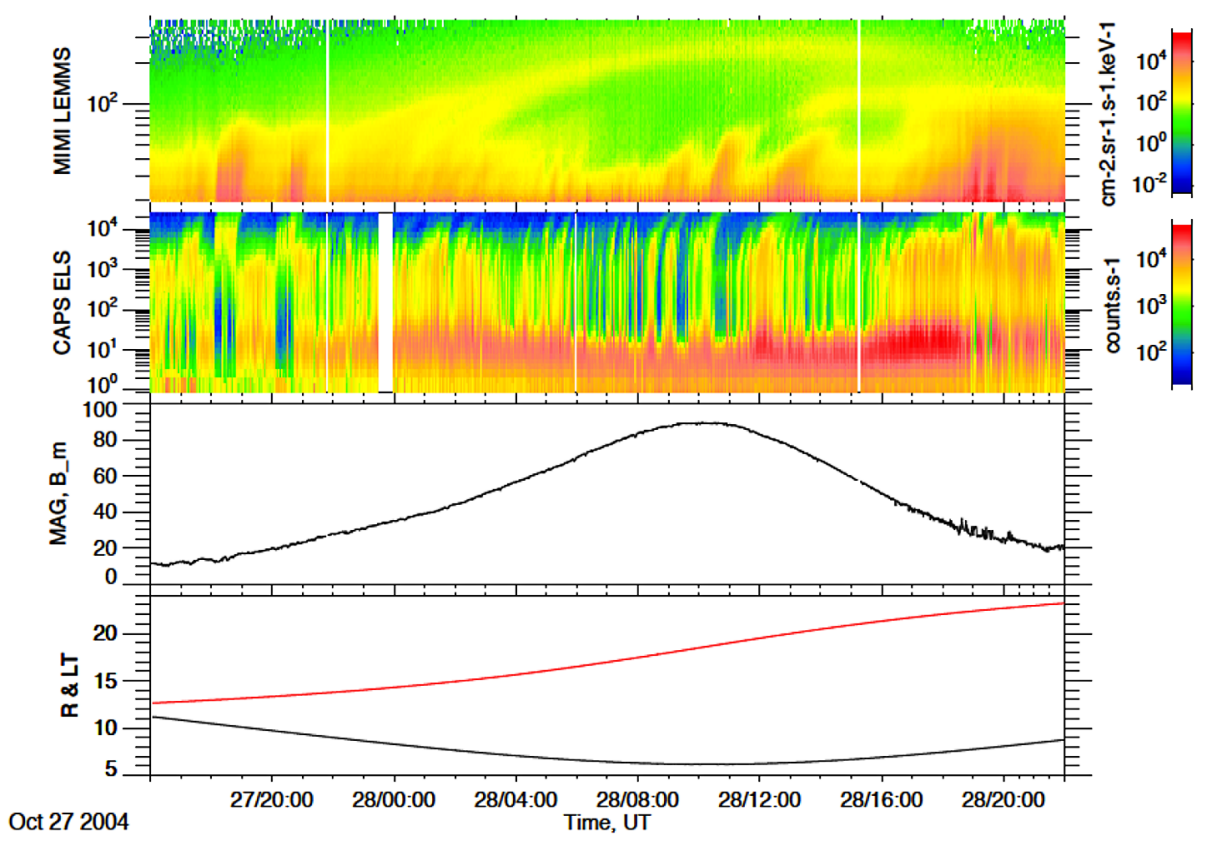

Fig. 15 Cassini multi-instrument observations of injection events showing the broad diversity of signatures encountered. From top to bottom: MIMI LEMMS electron energy-time spectrogram, CAPS ELS electron energy-time spectrogram, MAG magnetic field magnitude, Radial Distance and Local Time (in red). Figure made with the AMDA tool available at CDPP, http://cdpp.cesr.fr

the centrifugal interchange instability. This is because, for a given particle energy per unit charge, and a given $L$ value, the gradient/curvature drift is about 25 times faster at Saturn than at Jupiter (Hill et al. 2005), which thus makes Kronian injection events easier to detect.

Due to adiabatic gradient and curvature drifts, the injected plasma indeed exhibits significant longitudinal drift dispersion. On a linear energy-time spectrogram, an injection/dispersion event is revealed as a V-shaped structure, with hot ions (electrons) forming the left (right) legs of the $\mathrm{V}$. The apex of the $\mathrm{V}$ marks the original injection longitude, while the width of each leg indicates the width of the injection channel. The slope of the legs of the $\mathrm{V}$ is inversely proportional to the elapsed time since the original injection. Hence, by combining the original injection longitude and the elapsed time, the local time of the original injection can also be determined (Hill et al. 2005). Fewer, but spatially larger, events are observed at higher energies by the Cassini MIMI instrument (Mauk et al. 2005). These features are long-lived and dominate the appearance of the inner magnetosphere at medium and high energies (Fig. 15); they show a broad diversity of time-dispersed features produced by the combined effects of prograde (ions) and retrograde (electrons) magnetic drifts and the prograde 'ExB' drift (Paranicas et al. 2007). Over 100 injection/dispersion signatures have also been observed at Jupiter by the Galileo spacecraft. As at Saturn, the behaviour of the higher-energy particles at Jupiter showed much larger scale injections that are relatively long-lived (Mauk et al. 1999). These injections extended over $\sim 9-27 \mathrm{R}_{\mathrm{J}}$ and occurred at all longitudes and local times.

At Saturn, Chen and Hill (2008) and Chen et al. (2010) used Cassini plasma spectrometer observations and included 622 events in a statistical study of the smaller-scale injections. Their analysis revealed that the injections are spatially clustered within $\sim 5 \mathrm{R}_{\mathrm{S}}$, and also 

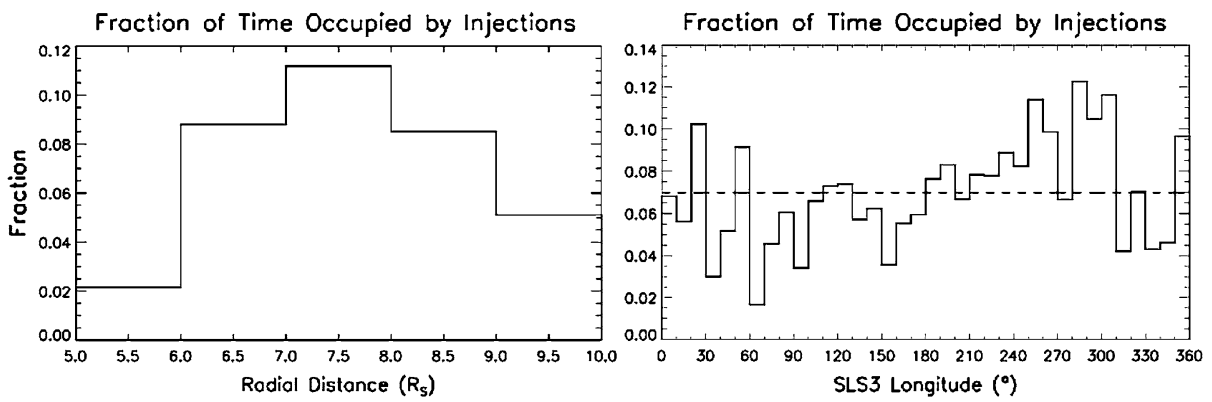

Fig. 16 Plasma inflow statistics at Saturn: (a) fraction of observation time occupied by plasma inflow channels within $1 \mathrm{R}_{\mathrm{S}}$ bins, averaged over SLS3 longitudes; (b) fraction of observation time occupied by plasma inflow channels within $10^{\circ} \mathrm{SLS} 3$ longitude bins averaged over the radial range $5-10 \mathrm{R}_{\mathrm{S}}$. The dashed horizontal line is the longitudinal average. Figures taken from Chen et al. (2010)

between $\sim 10-11 \mathrm{R}_{\mathrm{S}}$ (Fig. 16); their longitudinal widths seldom exceed $\sim 1 \mathrm{R}_{\mathrm{S}}$; their ages since formation seldom exceed the Saturn rotation period; and their spatial distributions in both longitude and local time are essentially random. The combined widths of all injection channels at any given time occupy only a small fraction (about $7 \%$ ) of the entire longitude space (Fig. 16). The inflow channels are thus evidently much narrower than the intervening outflow channels. If the total mass fluxes of the incoming hot plasma and outgoing cold plasmas are in balance (on average), then we would expect the ratio of inflow to outflow velocity to be approximately given by $V_{i} / V_{o} \sim\left(M_{c} / M_{h}\right)(\xi /(1-\xi))$, where $M$ denotes typical flux tube content of the plasma, subscripts indicate cold, outflowing (c) or hot, inflowing (h) populations, and the quantity $\xi \sim 7 \%$ is the fraction of the longitude space occupied in total by the inflowing events. The outflow event speeds are typically $\lesssim 1-2 \mathrm{~km} \mathrm{~s}^{-1}$ (Wilson et al. 2008), and so we expect the inflow speed to be at least $\sim 13$ times greater than this figure. Both inflow and outflow velocities appear to be smaller than the observed azimuthal velocity of the plasma, which is, in turn, lower than the velocity of planetary corotation. This ordering is consistent with theoretical considerations (Hill 2006). This observational finding is not generally predicted by theoretical models of the interchange process, in which the inflow and outflow channels are usually of roughly equal width. This discrepancy will be addressed in the next section.

Many injection events have relatively young ages, as discussed by Burch et al. (2005). The very young events were not included in the analysis of Chen et al. (2010), but a few of them have been studied in detail by Burch et al. (2005) and Rymer et al. (2007). Burch et al. (2005) used low-energy plasma measurements to estimate, for one particular event, an inward injection velocity of $25 \mathrm{~km} \mathrm{~s}^{-1}$ between $10 \mathrm{R}_{\mathrm{S}}$ and $5 \mathrm{R}_{\mathrm{S}}$, whereas Rymer et al. (2007) derived a radial injection speed of $71 \mathrm{~km} \mathrm{~s}^{-1}$ between $11 \mathrm{R}_{\mathrm{S}}$ and $7 \mathrm{R}_{\mathrm{S}}$ for another event. Whereas the latter estimate is outside the range of velocities inferred by Chen et al. (2010), the former is roughly consistent with their statistical results. More recently, Kennelly et al. (2013) conducted a statistical analysis of very young injection events, using observations from the Cassini Radio and Plasma Wave Science instrument. Whereas their occurrence rate with respect to $\mathrm{L}$ shell largely agrees with the distribution published by Chen et al. (2010), they do identify the post-noon (11-17 SLT) and near-midnight (19-3 SLT) regions as preferred sectors for the origin of injection events. At Jupiter, Russell et al. (2005) performed a statistical survey of the plasma-depleted flux tubes observed by Galileo in the Io torus (29 events). These flux tubes occupy $0.32 \%$ of the torus volume outside the orbit of Io. They proposed that the amount of magnetic flux transported by these thin flux tubes could supply 
the requisite amount of magnetic flux (mass-loaded) transported to the magnetotail provided the inward velocity is $\sim 300$ times that of the outward plasma motion. This represents a radial velocity of the order of $10 \mathrm{~km} \mathrm{~s}^{-1}$ in the torus.

\subsection{Comparing Simulations and Observations of Interchange}

Several numerical models have addressed the large-scale configuration and dynamics of giant plant magnetospheres, including those developed at the University of Michigan (e.g. Hansen et al. 2005; Jia et al. 2012) and at Nagoya/UCLA (e.g. Fukazawa et al. 2007, 2010). However, only a limited number of such codes have focused on the development of the interchange instability itself and how it is influenced by varying internal and external conditions. The rest of this section is devoted to those models which have been specifically used to investigate the interchange process. The main differences between models in this class are related to assumptions concerning rotational/interchange effects, plasma sources, current drivers, solar wind influence, and spatial regions of model validity.

\subsubsection{The Rice Convection Model for Jupiter and Saturn}

The Rice Convection Model is a multi-fluid physical model which was initially developed at Rice University in order to study solar wind-driven convection in the Earth's magnetosphere and the electromagnetic coupling to the ionosphere (e.g. Wolf 1983). This model was then adapted to simulate the rotation-dominated magnetosphere of Jupiter (Yang et al. 1992, 1994), and recently further extended to investigate the magnetosphere of Saturn (Liu et al. 2010).

The Rice model treats the three-dimensional problem of MHD flow in a planetary magnetosphere as a pair of two-dimensional problems: one in the equatorial plane of the magnetosphere, and the other in the planetary ionosphere. In this approach, the horizontal divergence of the magnetospheric current is balanced in the ionosphere by the analogous divergence of the Pedersen current, the two regions being connected by Birkeland currents flowing along magnetic field lines (Vasyliūnas 1970). In rapidly rotating magnetospheres, the centrifugal drift (or inertial) current is usually one of the major contributions to the total magnetospheric current, whereas, at the Earth, the major currents usually arise from a combination of magnetic gradient drift, magnetic curvature drift and the magnetization current associated with the magnetic moment of the gyrating plasma particles.

Early simulations from the Rice Convection Model applied to the Io torus at Jupiter focused on the influence of the radial width of the initial plasma distribution on the subsequent scale size of the dominant convection cells which emerge in the simulated flow. These simulations produced regularly spaced, long, thin 'fingers' of plasma outflow from the outer edge of the Io torus, interspersed with fingers of inflow from the surrounding magnetosphere (Yang et al. 1994). The plasma outflow in the fingers was suggested to be the main mechanism of plasma transport from the Io torus to the outer magnetosphere through the centrifugal interchange instability. The exponential growth rate of the instability was found to be proportional to the azimuthal wave number of the disturbance, and hence inversely proportional to the azimuthal scale size of the disturbance, in agreement with the analytical linear analysis of Huang and Hill (1991). The instability growth rate for a given wave number scales with the ratio of the total flux tube content divided by the ionospheric Pedersen conductivity.

A narrow, ribbon-like structure near Io's orbit is observed to be embedded within the larger torus. This 'ribbon' is a very dense, narrow annulus of plasma, situated around 
5.6-5.8 $\mathrm{R}_{\mathrm{J}}$. In order to understand the stability properties of this ribbon structure, $\mathrm{Wu}$ et al. (2007) decreased the size of the simulation region and increased the numerical grid resolution in order to resolve the smallest convection cells. They found that the dominant azimuthal scale of the interchange cells was about half of the radial width of the initial distribution that produced them. The small ribbon-scale structures thus grow faster than the larger torus-scale structures for given values of the flux tube content and ionosphere conductance. This observation emphasizes the importance of stabilizing mechanisms for maintaining the persistent torus structure, and calls for the inclusion in future simulations of ring current impoundment (e.g. Siscoe et al. 1981) and velocity shear (departure from planetary corotation, Pontius et al. 1998).

The relatively large growth rate for the ribbon in the Io torus also calls for the inclusion of the Coriolis and acceleration currents in the Rice Convection Model. This has been recently achieved for Saturn. Liu et al. (2010) have included the effects of the Coriolis force and the pickup current in order to study the plasma convection pattern in the inner magnetosphere of Saturn. To that purpose, a continuously active, distributed inner plasma source has also been added to the simulation. The plasma source model used in that initial simulation had a total mass-loading rate of only $24 \mathrm{~kg} \mathrm{~s}^{-1}$ and an ionospheric Pedersen conductance of 0.3 mho. The simulation confirmed that fast and narrow plasma inflow channels alternate with slower and wider outflow channels, in agreement with the Cassini observations of injection events described above. The Coriolis force was found to be responsible for: (i) the bending of the convection cells in the retrograde direction; (ii) the slowing of their growth; and (iii) the broadening of the outward-moving fingers. In addition, the inclusion of an active plasma source made the inflow sectors much narrower in longitude than the interspersed outflow sectors (Fig. 17). The simulation was then extended to include the effects of finite plasma pressure (i.e. cold plasma with finite temperature). The associated $\mathbf{J} \times \mathbf{B}$ force at Saturn was found to augment the driving provided by the centrifugal force and enhance by $50 \%$ the centrifugal instability growth rate (Liu and Hill 2012). The plasma source model used in the most recent simulation had a total mass-loading rate of $240 \mathrm{~kg} \mathrm{~s}^{-1}$ and the ionospheric Pedersen conductance was increased to 3-6 mho (simulation results not shown here). These incremental adaptations of the Rice Convection Model for Jupiter and Saturn have so far been generally successful in reproducing spacecraft observations of the centrifugal instability in giant planet magnetospheres. A multi-fluid model for Saturn's rapidly rotating magnetosphere is described in Sect. 5.

\subsection{Interchange in the Laboratory}

Interchange instabilities occur in a variety of natural plasma environments, not only the fast rotating magnetospheres of Jupiter and Saturn just described, but also at the plasmapause and in the F-layer of the ionosphere at Earth. They also arise in laboratory plasmas where magnetic confinement configurations can lead to pressure-driven interchange instability. Although the plasma beta in laboratory experiments is much lower than inside the Jovian or Kronian magnetospheres, observations of the structures resulting from the development of the artificial interchange instability may be relevant to the dynamical processes within giant planet magnetospheres. We briefly illustrate here the potential for such a comparison in the case of Saturn.

Evidence from multiple Cassini instruments exists for some sort of azimuthal asymmetry, moving in the corotational sense, thus causing asymmetric mass and energy flow around the planet (see a review by Carbary and Mitchell 2013). Such an asymmetry has been notably reported in the electron density determined by the Cassini Radio and Plasma Wave Science instrument in the inner magnetosphere (Gurnett et al. 

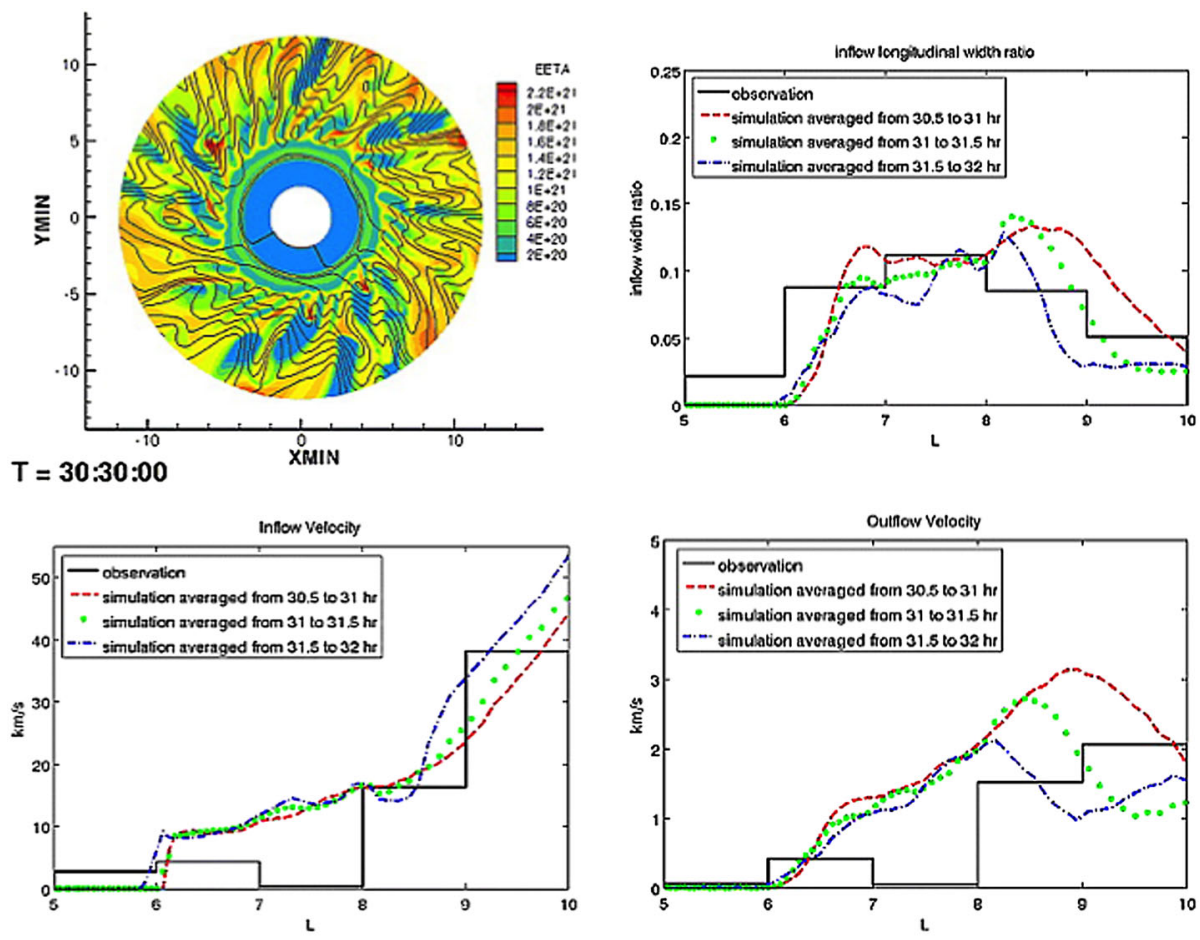

Fig. 17 Simulation results for the evolution of plasma convection in Saturn's inner magnetosphere, with the inclusion of the Coriolis force and an active plasma source. Top left: Colour indicates flux tube ion content (ions/Weber) projected on the equatorial plane, in the corotating frame of reference, with equipotential lines (flow streamlines) added at $15 \mathrm{kV}$ intervals. Top right: Fraction of the available longitude space occupied by inflow channels, compared with the observations from Chen et al. (2010). Bottom: Longitudinally averaged inflow and outflow velocities, compared with observations from Chen et al. (2010). Figures taken from Liu et al. (2010)

2007). One proposed explanation is a corotating convection pattern (Gurnett et al. 2007; Goldreich and Farmer 2007), initially proposed for the Jovian system (Vasyliūnas 1983; Hill et al. 1981). The proposed systematic pattern consists of outflow in an active sector and inflow in the complementary longitude sector (Fig. 18), and the pattern corotates with the planet, although the plasma may not. The resulting two-cell pattern may be generated spontaneously and maintained indefinitely via the centrifugal instability. As originally pointed out by Hill et al. (1981), the flux-tube interchange instability may initiate the plasma outflow, and the proposed corotating convection pattern may represent the steady state toward which the system evolves, i.e. the saturation state of the centrifugal interchange instability.

The two-cell convection pattern has also been observed to extend along a spiral path in the outer magnetosphere of Saturn (Burch et al. 2009). Most previous models of centrifugally driven convection, however, suggest that the convective motions should be dominated by high-order $(m \gg 1)$ azimuthal modes that evolve into azimuthally confined fingers, as discussed in the previous section. Gurnett et al. (2007) nevertheless suggested that the lowest-order, $m=1$ (two-cell) mode should dominate in the Enceladus neutral torus, since this mode produces the longest path length through the source regionthereby giving the largest density increase and the largest growth rate for the instability. How the centrifugal instability maintains a single longitudinal asymmetry $(m=1$ 

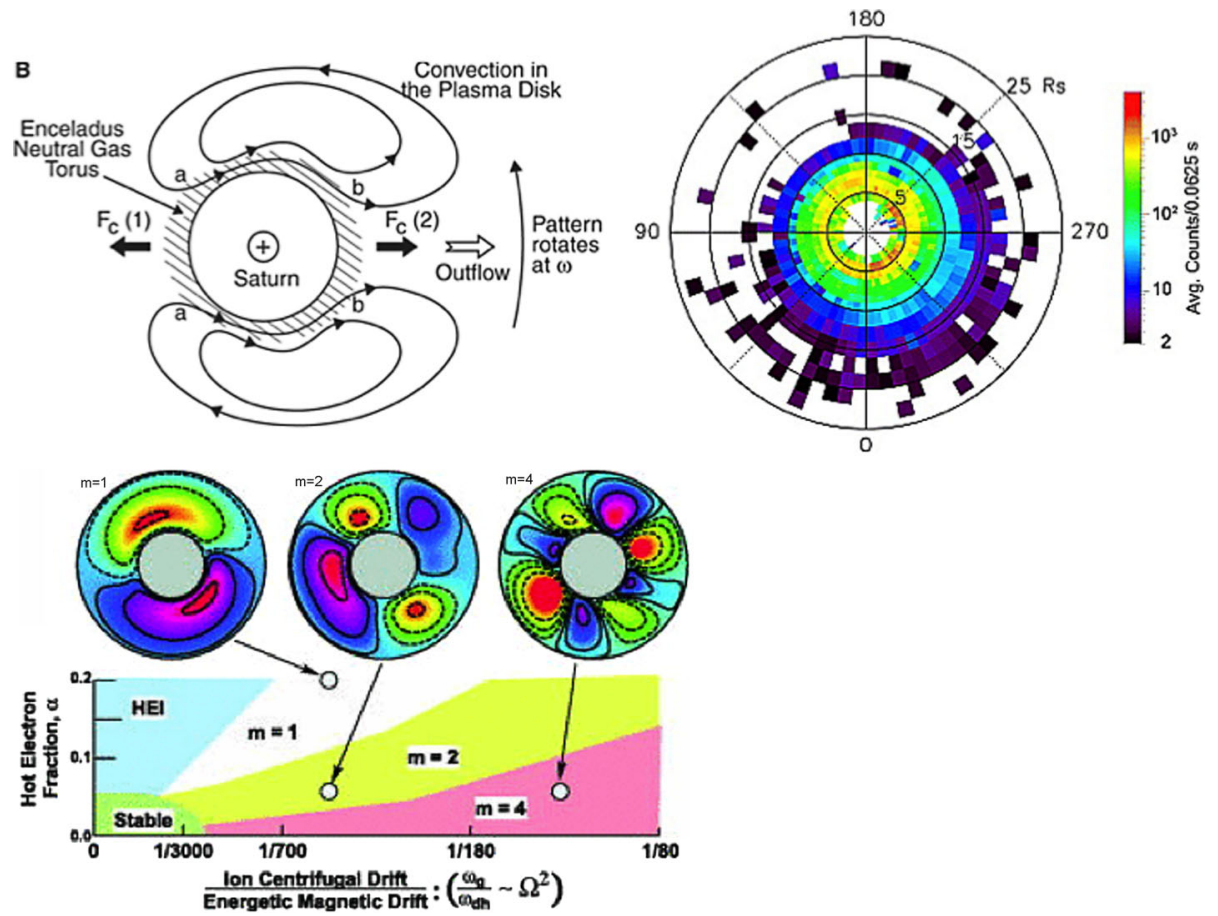

Fig. 18 Top left: Proposed two-cell corotating convection pattern in Saturn's inner magnetosphere. Figure from Gurnett et al. (2007), reprinted with permission from AAAS; Top right: Average ion counts organized against SLS3 longitudes. Figure from Burch et al. (2009); Bottom: Computed variation of the interchange mode structure as the fraction of energetic electrons and the plasma rotation rate $(\Omega)$ change. Only the regimes dominated by $m=1$ and $m=2$ are observed experimentally. Reprinted with permission from Levitt et al. (2005b). Copyright Physics of Plasmas 2005, AIP Publishing LLC

mode) rather than multiple asymmetries, or how the dominant $m=1$ mode can persist on decadal time scales are questions which remain unanswered. Additional challenges for such models are presented by the observation of two distinct oscillation periods in Saturn's magnetospheric field and kilometric radiation (e.g. Andrews et al. 2012; Lamy 2011; Gurnett et al. 2009).

Whereas the interchange driven by energetic electron pressure has been studied in detail in artificial plasmas, the excitation of the centrifugal interchange instability in a laboratory plasma confined by a magnetic dipole has been reported by Levitt et al. (2005a). These first experimental observations of the centrifugally driven interchange mode were made in the Collisionless Terrella eXperiment (CTX) (dipole-confined plasma) by inducement of $\mathbf{E} \times \mathbf{B}$ rotation, through application of a radial electric field. Both centrifugally driven interchanges, and interchanges driven by hot electron pressure, were simultaneously observed in the same discharge experiment. The observed mode structures for these instabilities are quasi-coherent in the laboratory frame of reference and are dominated by low azimuthal $m$ numbers and broad radial structures. The dispersive properties of the global, coherent modes are modified by the presence of the hot electron population, which causes long wavelength modes to be more unstable: drift resonant particles induce polarization currents which preferentially stabilize higher $m$ modes (Levitt et al. 2005b). For a relatively low fraction of hot electrons and rapid plasma rotation, the most unstable interchange mode has a shorter 
wavelength, $m=4$, and grows with nearly zero real frequency in the rotating frame. As the rotational driver is reduced or as the energetic electron population increases, the azimuthal mode number $m$ decreases and the interchange mode acquires a real frequency in the direction of the electron magnetic drift. The radial phase has a significant shift of $\pi / 2$ for the dominant $m=1$ mode. The corresponding fluctuations presented a broad azimuthal spiral structure, which appeared to rotate rigidly in time. Figure 18 displays the interchange mode structure as a function of the plasma rotation rate and the fraction of energetic electrons.

In this context, energetic electrons also constitute an important population of particles in the magnetospheres of Jupiter and Saturn. For a given ion mass $M_{i}$ and energetic electron energy $E_{h}$, the ratio of the gyrofrequency and drift frequency, denoted $\omega_{g} / \omega_{d}$, is proportional to $\left(1 / 2 M_{i} \Omega^{2} L^{2}\right) / 3 E_{h}$, where $L$ is the equatorial radius of the field line and $\Omega$ is the angular velocity of the plasma. For the experiments described in Levitt et al. (2005a), $\omega_{g} / \omega_{d} \sim 1 / 100$, whereas the same ratio is $1 / 55$ at Saturn (and 1/45 at Jupiter). The mode structure that results from the interaction of the centrifugal instability with energetic particles, and which is observed in these laboratory experiments, bears a striking similarity with some of the Cassini observations reported in Saturn's magnetosphere. The detailed comparison of these two systems, however, requires further studies to be initiated.

\section{Magnetodisc-Plasma Interactions}

\subsection{General Properties}

As noted in Sect. 1, the overall position of the plasmadisc and magnetodisc are determined by the plasma velocity, mass density, and the magnetic field. In the outer planets, especially Jupiter and Saturn, the energy and momentum transport is controlled by three influences: (a) solar wind and interplanetary magnetic field (IMF), (b) the planet's rotation and plasma sources, and (c) interactions with the planet's satellites. The influence of these processes is shown in Fig. 19. The interaction of the solar wind with the magnetic field of the planet produces a bow shock and magnetopause that define the outer boundary of the magnetosphere. Along the flanks and in the deep (several tens of planetary radii) tail, solar wind drives plasma flows that are predominantly down-tail. In the middle magnetosphere a twocell convection pattern, particularly for antiparallel interplanetary magnetic field (IMF), can develop where there are induced sunward plasma flows, some of which are associated with reconnection events. Imposed on these flows is the rotation field set up by the planet and its magnetic field. For the inner planets the rotational effects tend to be small and most dominate out to a few planetary radii, at most. At the outer planets the rotational effects can be much stronger, dominating out to several tens of planetary radii. This effect is strongest within the Jovian magnetosphere, though it is also a strong aspect of the dynamics of the Kronian magnetosphere.

In the presence of a two-cell convection pattern, the convection velocity in the inner to middle magnetosphere tends to be enhanced on the dawn side and reduced on the dusk side. This modulation of the convection speed, as discussed later, has an important effect on the transport of energy and mass within the magnetosphere.

The addition of the solar wind and rotational influences to create the overall, average plasma convection flows is shown in Fig. 20 and illustrates some aspects of the Vasyliūnas convection pattern (Vasyliūnas 1983). Co-rotational flows exist in the inner magnetosphere, while beyond the Alfvén radius in the outer magnetosphere, tailward flows prevail. In between these regimes, there exists a region of transition in the middle magnetosphere with the direction of flow strongly dependent on the prevailing solar wind conditions. 
Fig. 19 Schematic of the equatorial velocity fields within the magnetospheres of the outer planets

Fig. 20 Schematic of general plasma convection with the magnetospheres of the outer planets (Bagenal and Delamere 2011)
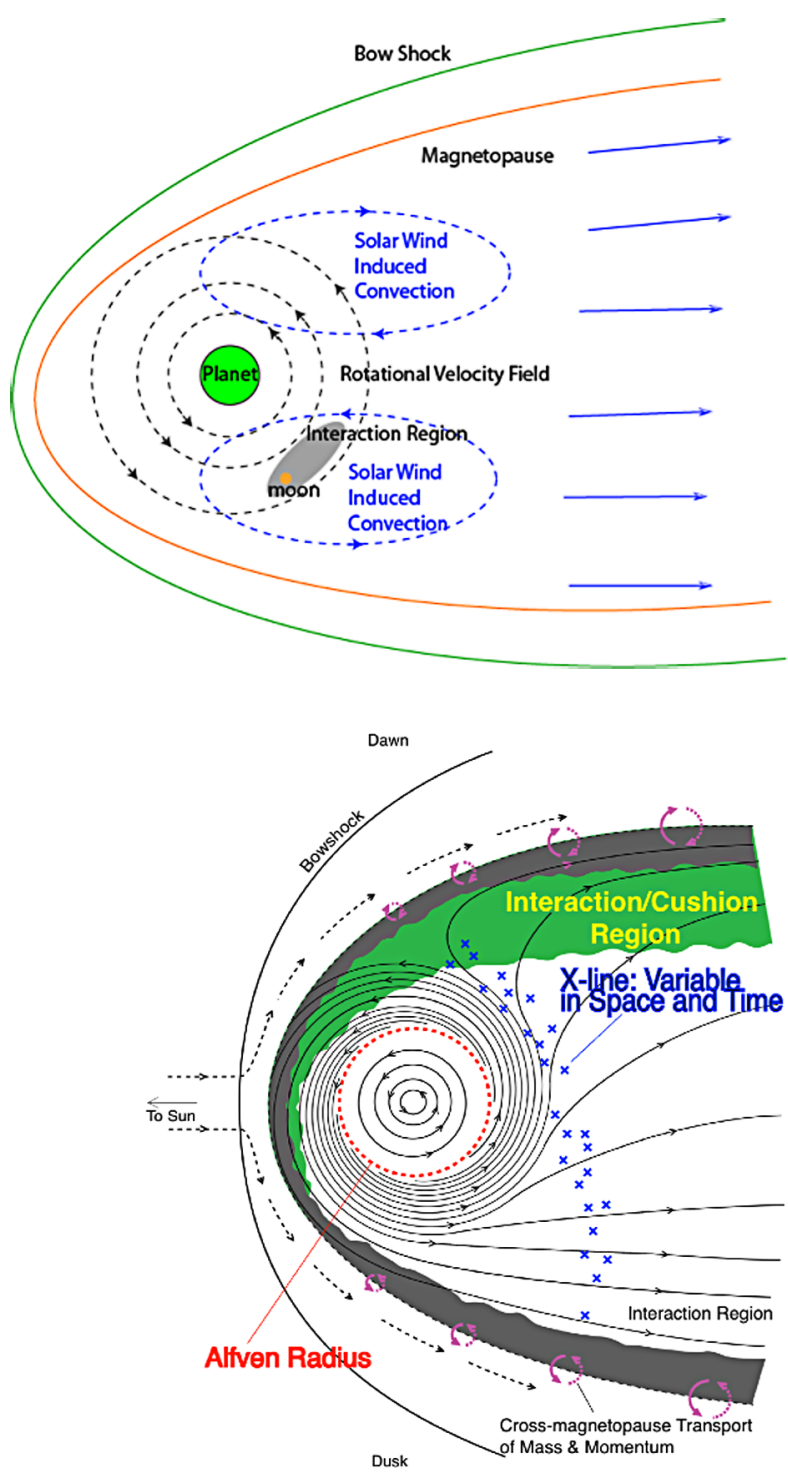

The overall convection pattern in Fig. 20 is not stable, and is subject to an interchange instability where there are alternating longitudinal regions of outward moving cold dense plasma and inward moving hot tenuous plasma (Pontius et al. 1986; Hill et al. 2005, Sects. 3 and 4). Injections of energetic particles into the inner magnetosphere are seen in both the Jovian (Mauk et al. 1997) and Kronian (Mauk et al. 2005) magnetospheres.

Transport of energy and mass is also modified by the presence of moons in the inner magnetosphere. At Jupiter, the strongest interaction is produced by Io, but both Europa and Ganymede have sufficient influence that aurorae can be seen at their magnetic footpoints in the Jovian ionosphere (e.g. Clarke et al. 1998). At Saturn, analogous footpoint emissions from Enceladus have been observed (Pryor et al. 2011). Footpoint emissions associated with Titan have yet to be identified, however the motion of Titan itself has the potential for 
modulating Saturn's kilometric radiation (SKR) — a phenomenon which is associated with the auroral process (Menietti et al. 2007).

The moons associated with footprint aurorae can generally produce plasma densities that, at least locally, can exceed the density of the ambient plasma in the magnetodisc. Moreover, the plasma tied to these moons initially moves at Keplerian orbits when it is first created through ionisation of neutral species. Since the Keplerian speed tends to be much slower than the convection of the ambient plasma, the moon-magnetosphere interaction produces a 'drag' effect on the ambient plasma. As discussed in Sect. 2 and in the following subsections, there is also potential for global effects developing from these moon-magnetosphere interactions. It is also interesting to note that these interactions can occur in sub-Alfvénic and/or subsonic flows, such that the presence of the moon in question can be 'felt' by the plasma in the region upstream of the moon itself. In the following subsections we provide additional details on these processes as applied to Saturn where there is a wealth of data from the Cassini mission (e.g. Blanc et al. 2002).

\subsection{Plasmoid Formation at Saturn}

Prior to 2011, only a handful of definitive plasmoids had been detected at Saturn by Cassini (Jackman et al. 2009; Hill et al. 2008). These reconnection-related events are seen at distances $40-50 \mathrm{R}_{\mathrm{S}}$ from the planet's centre, with a recurrence rate of $\sim 5-7$ days. During two of these events, Cassini was near the centre of Saturn's current sheet and observed a plasmoidal composition dominated by water group ions, strongly suggesting that plasmoid formation is often linked to processes occurring in the inner magnetosphere. A more detailed analysis of the Cassini deep tail orbits in 2006 showed the presence of a total of 34 plasmoids, with an observed occurrence rate of one plasmoid every 2.4 days (Jackman and Arridge 2011).

Another sign that the magnetotail is unstable is the appearance of quasi-periodic flapping of the plasma sheet in this region. This flapping was originally interpreted as the periodic passage of plasmoids by Burch et al. (2009). A far more likely interpretation attributes the flapping to the global magnetospheric oscillations observed in the Saturn system (Jackman et al. 2009; Arridge et al. 2011).

Global MHD modeling has been used to examine the overall shape of the Kronian magnetosphere (Fukazawa et al. 2007) and showed the development of the Kelvin-Helmholtz instability on the flanks during periods where the IMF and planetary equatorial fields are parallel, and the development of magnetic reconnection during periods where these fields are antiparallel. Zieger et al. (2010), using their MHD model, demonstrated that quasi-periodic reconnection, between closed field lines in the tail, could be driven with periods between 20-70 hours, depending on the value of the upstream solar wind's dynamic pressure. Global multi-fluid simulations have been also been performed by Kidder et al. (2012) in order to investigate plasmoid formation. There is scope for improving the inner boundary conditions related to ion density in these multi-fluid calculations, in light of additional plasma moment determinations from the Cassini plasma spectrometer experiment (e.g. Thomsen et al. 2010). The multi-fluid approach has the advantage over single-fluid MHD of being able to separately track the dynamics of the different ion species and sources, instead of simply calculating their average properties. The different components in the multi-fluid model include (a) solar wind protons, (b) ionospheric protons, (c) water group ions from Enceladus and (d) heavy ion group that can originate from either Enceladus or Titan. Due to the difference in mass and source, these different ion species can have different trajectories through the magnetosphere, and thereby have very different transport properties to those 

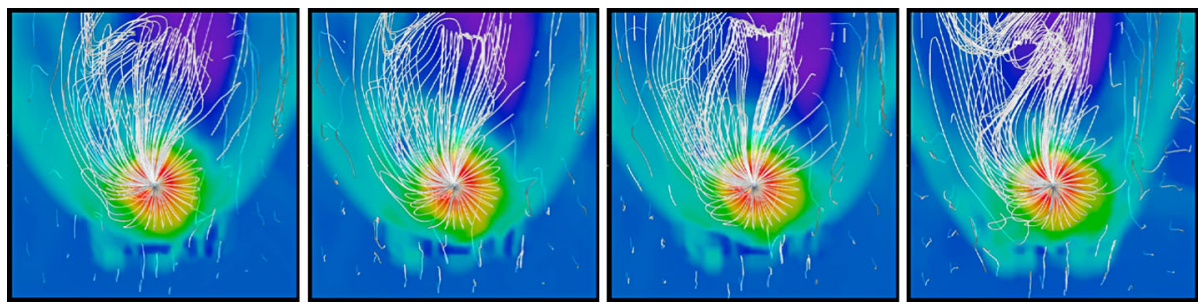

Fig. 21 Development of a plasmoid which includes loop-like structure as seen from a top view (from Kidder et al. 2012)

which would be evident in a one-fluid simulation. The development of a plasmoid in the multi-fluid model, which includes loop-like structures, is shown in Fig. 21. The skewing of the plasmoid towards the dawn sector arises from the relatively fast rotation of Saturn. While external triggering from IMF conditions is possible, the long time scale probably means that this type of triggering is probably not very common. Solar wind pressure pulses, however, can more efficiently produce reconnection and plasmoid formation. The composition of the plasmoid from the model was demonstrated to be primarily water group ions, consistent with the Cassini observations, and thus suggests that there is strong coupling between the inner and middle magnetospheres.

\subsection{Interchange Instability}

The dynamics of the inner magnetosphere, as noted above and in Sect. 4, are complicated by the potential growth of the interchange instability. The presence of this instability was detected very early in the data from Cassini (Burch et al. 2005; André et al. 2005). The first simulations of the interchange instability within a global Saturnian model were developed by Kidder et al. (2009) using the multi-fluid approach. An example of the simulated development of the interchange instability is illustrated in Fig. 22 which shows the evolution of equatorial plasma density. The growth and development of a single, outwelling interchange 'finger' is labeled with an asterisk. The development of the interchange instability is similar to that of the Raleigh-Taylor instability, in which a relatively dense fluid (in this case water group ions) sits over a less dense fluid (in this case solar wind protons), with the free energy being provided by the centrifugal force associated with the rotation of the plasma. The interchange instability starts as small ripples along the plasma density in the inner magnetosphere, where the density of the water group ions (designated $\mathrm{CNO}^{+}$ here) is relatively high. The ripples start to grow on the dawnside, reaching almost to the magnetopause as they pass through the midday sector. As they move into the dusk sector, components of the interchange structures can break off and move down the tail while, in the inner magnetosphere, they can become truncated, with the potential for regrowth during the next rotation about the planet. As a result, there is much more substantial outward transport of mass from the inner magnetosphere than suggested by the simple picture of Fig. 20.

The corresponding temperature profile is shown in Fig. 23. The plasma in the outer regions is hottest at several tens of $\mathrm{keV}$, while the plasma about the Enceladus torus is only at a few $\mathrm{eV}$. As the water group ions move out, they experience some heating and approach temperatures similar to that of the original magnetospheric plasma. Conversely, between the interchange fingers, hot tenuous magnetospheric plasma is seen to penetrate inwards. Thus the interchange instability is also important for energy transport within the magnetosphere 

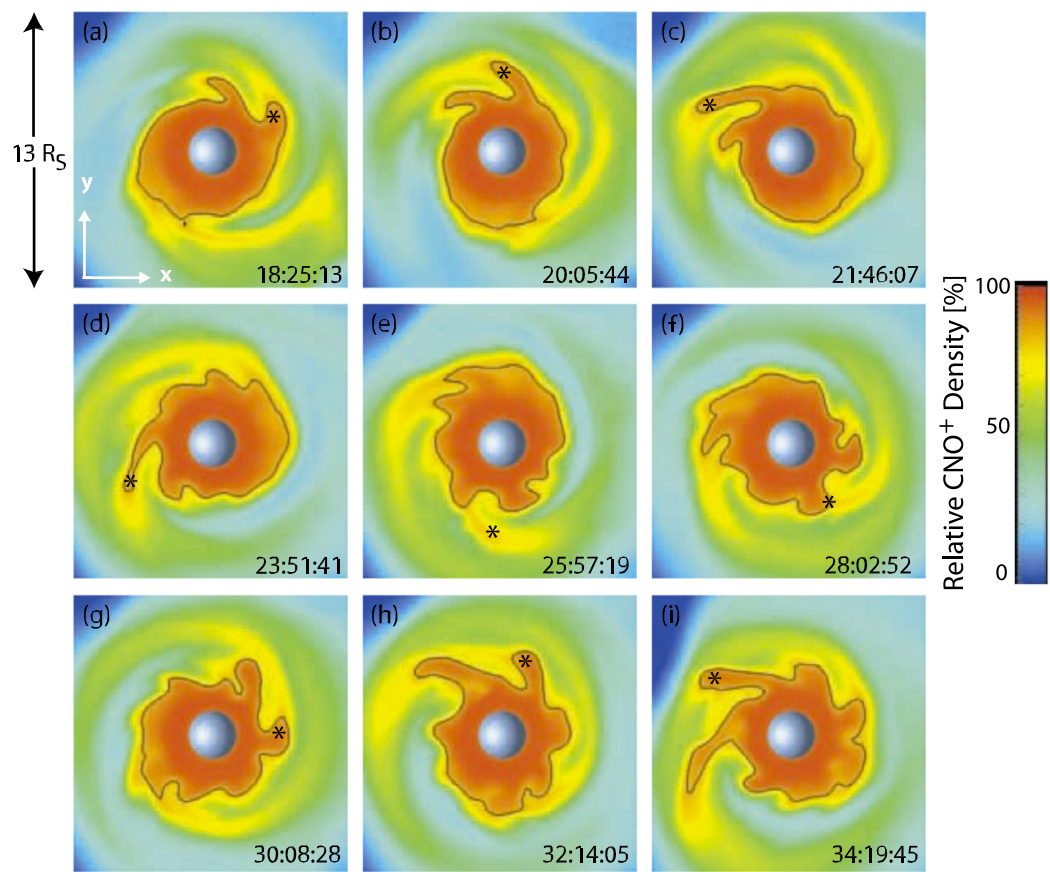

Fig. 22 Equatorial profile of the relative density of the $\mathrm{CNO}^{+}$ions originating from Saturn. The growth of the interchange instability is illustrated by the asterisk which marks the growth of one individual 'finger' (from Kidder et al. 2009)

and the spacecraft cutting through these fingers will see alternative regions of hot, tenuous and cold, dense plasma regions.

A particularly interesting feature seen in Figs. 22 and 23 is that the tip of the interchange fingers lead the finger itself. This effect arises from three-dimensional features of the instability. While much of the forcing is in the equatorial plane, the field lines themselves are tied to the planet at higher latitudes. In the original simulations by Kidder et al. (2009), the plasma density near Enceladus was not fully established by observations and a density nearly an order of magnitude too small was assumed. In this case, the high-latitude components also contributed to the interchange motion and, because of the additional centrifugal acceleration, the tip of the interchange finger leads the motion of the rest of the structure. The relevant point from these initial simulations is that the Kronian magnetosphere is unstable to the interchange instability over quite a wide range of the parameter space.

Subsequent simulations by Snowden et al. (2011a, 2011b) and Winglee et al. (2013) have utilized higher densities for the near-Enceladus plasma. As a result, the previous, forward curvature of the interchange figures is replaced with fingers that tend to have backward curvature (i.e. they lag the bulk finger motion). However, the difference in the velocity field between the dawn and dusk sides (c.f. Fig. 22) still causes the fingers to partially accelerate on the dawn flank and decelerate on the dusk flank, indicative of strong transport occurring where there is strong coupling between rotational and solar wind-related effects. Kidder et al. (2009) also demonstrated that the simulated growth rate of the instability could be enhanced by increases in the solar wind dynamic pressure and/or antiparallel IMF. In both cases, the convective electric field enhances the plasma velocity on the dawnside and thereby brings the plasma closer to the required velocity threshold associated with the 


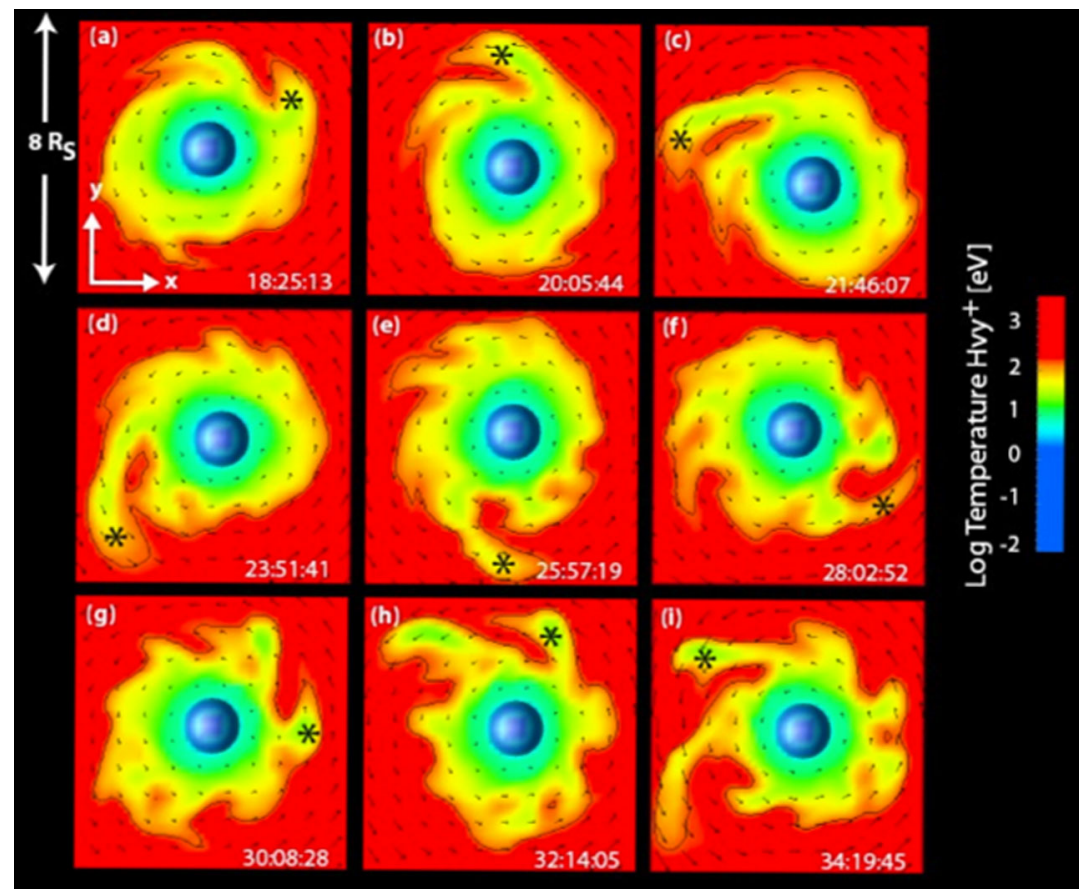

Fig. 23 The temperature profile corresponding to Fig. 22. Threading between the outward moving cold fingers is hot tenuous plasma from the middle magnetosphere (from Kidder et al. 2009)

growth of the instability. These results further emphasize the coupled nature of the problem, and that schematics such as Fig. 20, while providing a useful global picture, may be an oversimplification of the very important processes occurring deeper within the magnetosphere.

\subsection{Moon-Magnetosphere Interactions}

The simulated interchange fingers described above are sufficiently large that they are able to move beyond the orbit of Titan. The interaction of the Kronian plasma with Titan is known to produce an induced magnetosphere about Titan itself. The characteristics of the induced magnetosphere have been examined using local models, including MHD codes (Nagy et al. 2001; Kopp and Ip 2001; Ma et al. 2004), hybrid codes (Brecht et al. 2000; Ledvina et al. 2004; Simon et al. 2007) and multi-fluid simulations (Snowden et al. 2007). In the latter, the ion tail at Titan was demonstrated to be several Saturn radii in length, which demonstrates that Titan's induced magnetosphere is not an insignificant contributor of plasma to the Kronian magnetosphere in which it is embedded.

Given the potential for feedback between Titan's ion tail and the Kronian magnetosphere, multiscale/multi-fluid simulations that resolve the induced magnetosphere around Titan, as well as the main features of the Kronian magnetosphere, were developed by Winglee et al. (2009). In these initial simulations, time scales of a few tens of hours were considered and, due to the limited time scale, the refinement grid around Titan was held in a fixed position. An example of the results is shown in Fig. 24, where isosurfaces of the plasma density are shown over a region of about $15 \mathrm{R}_{\mathrm{S}}$ square. Prior to the arrival of an interchange finger, 


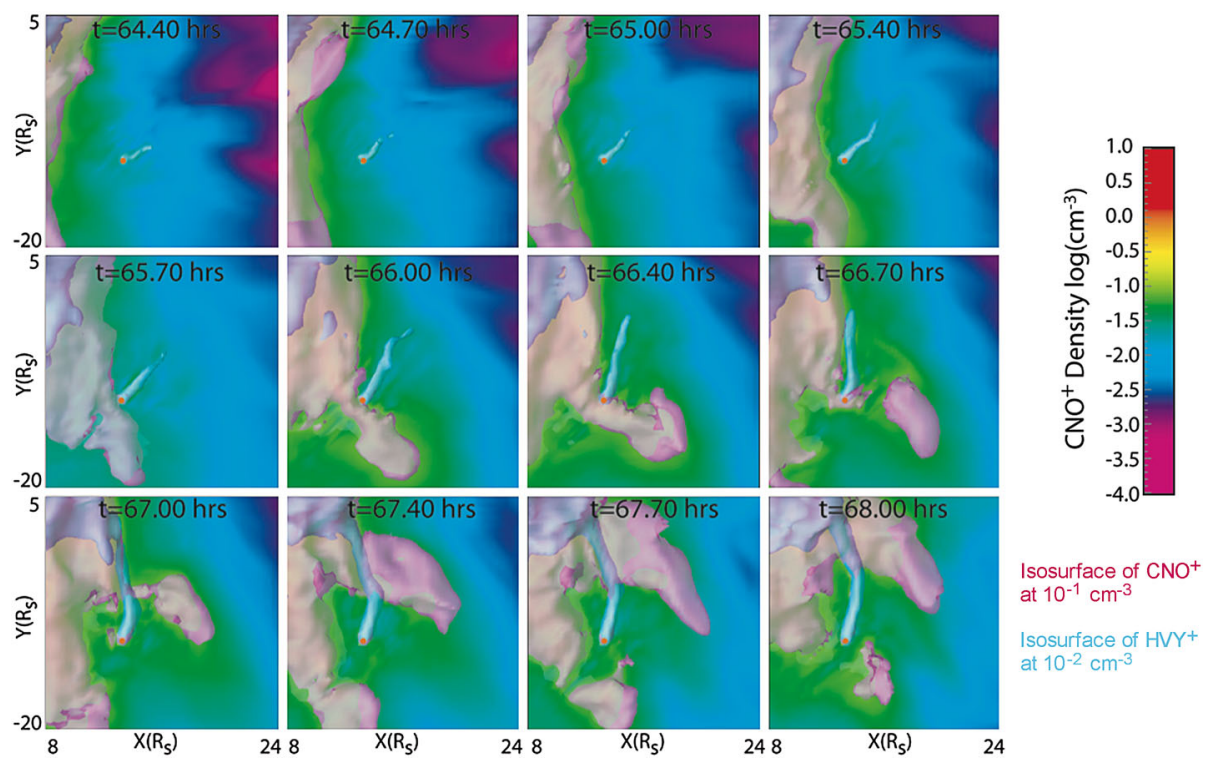

Fig. 24 Titan interacting with an interchange finger, derived from a multiscale global simulation that includes the Kronian magnetosphere and a refinement grid that includes the induced magnetosphere about Titan, with resolution of a few hundred kilometres. Interaction with the ambient plasma causes the flapping of Titan's ion tail and disruption of the near-Titan interchange finger (from Winglee et al. 2009)

Titan's ion tail is seen to lie along the average flow direction expected from the flow patterns in Fig. 20. However, as an interchange finger approaches and passes by Titan, the ion tail direction is substantially altered and moves to point more closely towards the planet. This interaction was also shown to substantially increase the outflow of plasma from Titan by nearly a factor of four. At the same time, there is the back reaction on the properties of the interchange finger, which is seen in the second and third rows of Fig. 24. In particular, the fingers in the vicinity of Titan can be disrupted or broken apart and this effect can occur upstream of the moon. This type of interaction can thus produce modulation of the physical properties of these fingers, as well as of the corresponding plasma mass transport in the Saturn system. In the example in Fig. 24, Titan was in the pre-midnight sector. Simulations that include Titan at pre-noon and post-noon sectors show that this effect of finger disruption is still very strong (Snowden et al. 2011a, 2011b). In the pre-noon sector, Titan's ion tail was sufficiently dense that it could prevent the movement of the magnetopause past Titan during IMF reversals. In the post-noon sector, slow convection of plasma past Titan can lead to the magnetic field being frozen in for periods as long as 15 minutes after IMF field reversals occur. These results again support the fact that a moon with an induced magnetosphere can have important impact on global mass and energy transport within its parent planetary magnetosphere.

\subsection{Interaction Between Global and Induced Magnetospheres}

The above interaction between the interchange fingers and the induced magnetosphere about Titan has typically been considered only a local interaction, with little consequences for the global dynamics of the Kronian magnetosphere. This paradigm has recently been brought into question by Winglee et al. (2013), who undertook simulations of the interaction for a 
full rotation of Titan about Saturn. This interaction was shown to have global consequences for the magnetosphere and could make a significant contribution to establishing the periodic signatures seen in many of the observable features of the Kronian magnetosphere (Carbary and Mitchell 2013).

Periodic modulation of Saturn's auroral kilometric radiation (SKR) was observed during the passage of Voyager 1 and used to determine the rotation period of the planet at $10 \mathrm{~h} 39 \mathrm{~min} 24 \mathrm{~s} \pm 7 \mathrm{~s}$ (Desch and Kaiser 1981). With the arrival of Cassini 24 years later, a slightly shifted period of $10 \mathrm{~h} 45 \mathrm{~min} 45 \mathrm{~s} \pm 36 \mathrm{~s}$ was determined (Gurnett et al. 2005; Kurth et al. 2008). In addition to a variability of the underlying period, there also appears to be a difference in the periods between the northern hemisphere $(\sim 10.6 \mathrm{hrs})$ and the southern hemisphere at $(\sim 10.8 \mathrm{hrs})$ (Gurnett et al. 2009). The difference in these two rates appeared to converge about seven months after Saturn's equinox in 2009.

The presence of this type of periodicity was a surprise, since the planet's internal magnetic field is highly aligned with its rotational axis. The fact that the period drifts with time suggests that its origin cannot be due to planetary rotation alone. The different periods between the northern and southern hemispheres further complicate any potential explanation of their physical origin. The same quasi-periodic modulation is observed in many of the properties of the Kronian magnetosphere, including the magnetic field (Espinosa and Dougherty 2000; Giampieri et al. 2006), low-energy plasma (Gurnett et al. 2007), energetic charged particles (Carbary et al. 2007), energetic neutral atoms (Paranicas et al. 2005; Carbary et al. 2008a), and the position of the magnetopause (Clarke et al. 2010). Whatever the source of the periodicity may be, it has global consequences for the energy and mass transport within the Kronian system.

The results from the previous section have already demonstrated that the interaction of Titan with an interchange finger causes the flapping of Titan's ion tail and that the interchange finger itself is also modified by the interaction. An important part of this interaction is that Titan's ion tail is seen to be pulled inwards by the interaction with the interchange finger. This means that there is partial reflection of energy and mass that would have otherwise propagated beyond Titan. In so doing, the system has the potential to set up a kind of resonance that favours the growth of modes of the interchange instability that have an apparent frequency near that of the planetary rotation, though the individual fingers themselves rotate with a period much longer than the planetary period. The buildup of this resonance was demonstrated in the long duration simulations of Winglee et al. (2013) and is illustrated in Fig. 25. In this simulation, an equilibrium for the global Kronian magnetosphere is established by running the code for 40 hours in the absence of a simulated Titan. After this period, Titan is then inserted with an initial position at the dusk terminator. The equatorial velocity in the top panels of Fig. $25 \mathrm{a}-\mathrm{c}$ show that, within about $7 \mathrm{R}_{\mathrm{S}}$, the flows are approximately rotational. At larger distances the flow pattern is modified by the superposition of a two-cell convection pattern which enhances the plasma velocities on the dawnside and reduces them on the duskside. The Titan obstacle produces a slowdown in the plasma velocities for a few hours in local time about its simulated orbital position. Because conservation of magnetic flux is required there is enhancement of the azimuthal velocity at smaller radial distances. This effect demonstrates that Titan's induced magnetosphere has both local and global consequences for the Kronian system's dynamics.

The simulated interchange instability at earlier times has five to seven large interchange fingers of plasma, similar to the result of Kidder et al. (2009), except that, with higher equatorial plasma density, the interchange fingers are curved in the backward direction (e.g. Fig. 25d). However, at later simulated times in Fig. 25e, f, the number of large interchange fingers is reduced to only three or four. Thus we have a configuration in which a rotationally 

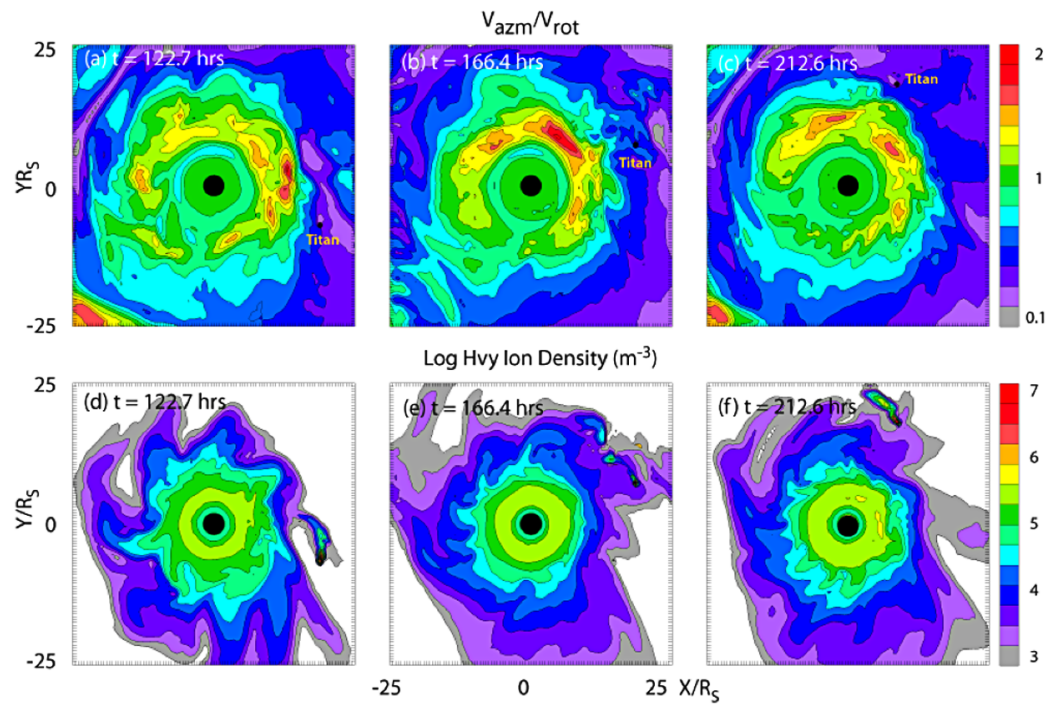

Fig. 25 Top panels show the azimuthal velocity in the equatorial plane while the lower panel shows the $\mathrm{Hvy}^{+}$ion density to indicate the positions of the interchange fingers, the position of Titan, and Titan's ion tail. Corotation dominates out to a distance $\sim 7 \mathrm{R}_{\mathrm{S}}$. This region is then followed by a sector where a two-cell convection pattern is imposed on the rotation field (see text). Titan suppresses the convection velocities over a few hours of local time in its vicinity (from Winglee et al. 2013)

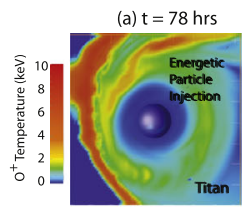

(f) $\mathrm{t}=88 \mathrm{hrs}$

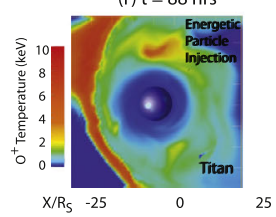

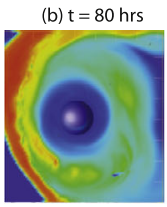

(g) $\mathrm{t}=90 \mathrm{hrs}$

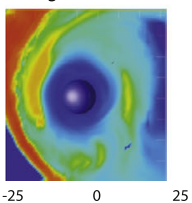

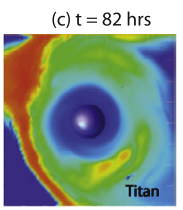

(h) $\mathrm{t}=92 \mathrm{hrs}$

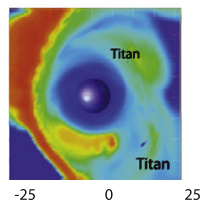

(d) $\mathrm{t}=84 \mathrm{hrs}$

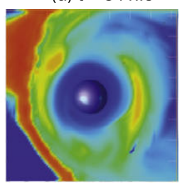

(i) $\mathrm{t}=94 \mathrm{hrs}$

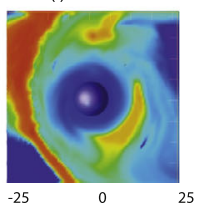

(e) $\mathrm{t}=86 \mathrm{hrs}$

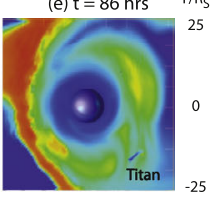

(j) $t=96$ hrs

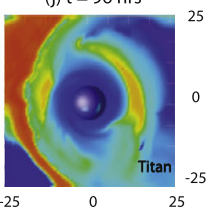

Fig. 26 The equatorial $\mathrm{O}^{+}$temperature during an energetic particle injection event that is tracked over approximately two Saturn rotations. The panels are arranged so that approximately a full period is shown in each row. Moving down the columns one can see the profiles separated by about eight planetary periods. Similar features in the energetic particles separated by a planetary period are apparent, although the particles themselves are actually different (from Winglee et al. 2013)

driven instability is being modified by an external influence that damps out the higher frequency components. For an observer at a fixed position, this has an interesting consequence which is illustrated in Fig. 26. This figure shows a simulated injection event tracked over two rotation periods. Titan is seen to provide herding of these energetic particles into the inner magnetosphere (top row) and produces some additional heating as they pass by Titan in their second passage around Saturn (bottom row). A second group of energetic particles is launched into the system in near-antiphase with the initial injected population; this effect 

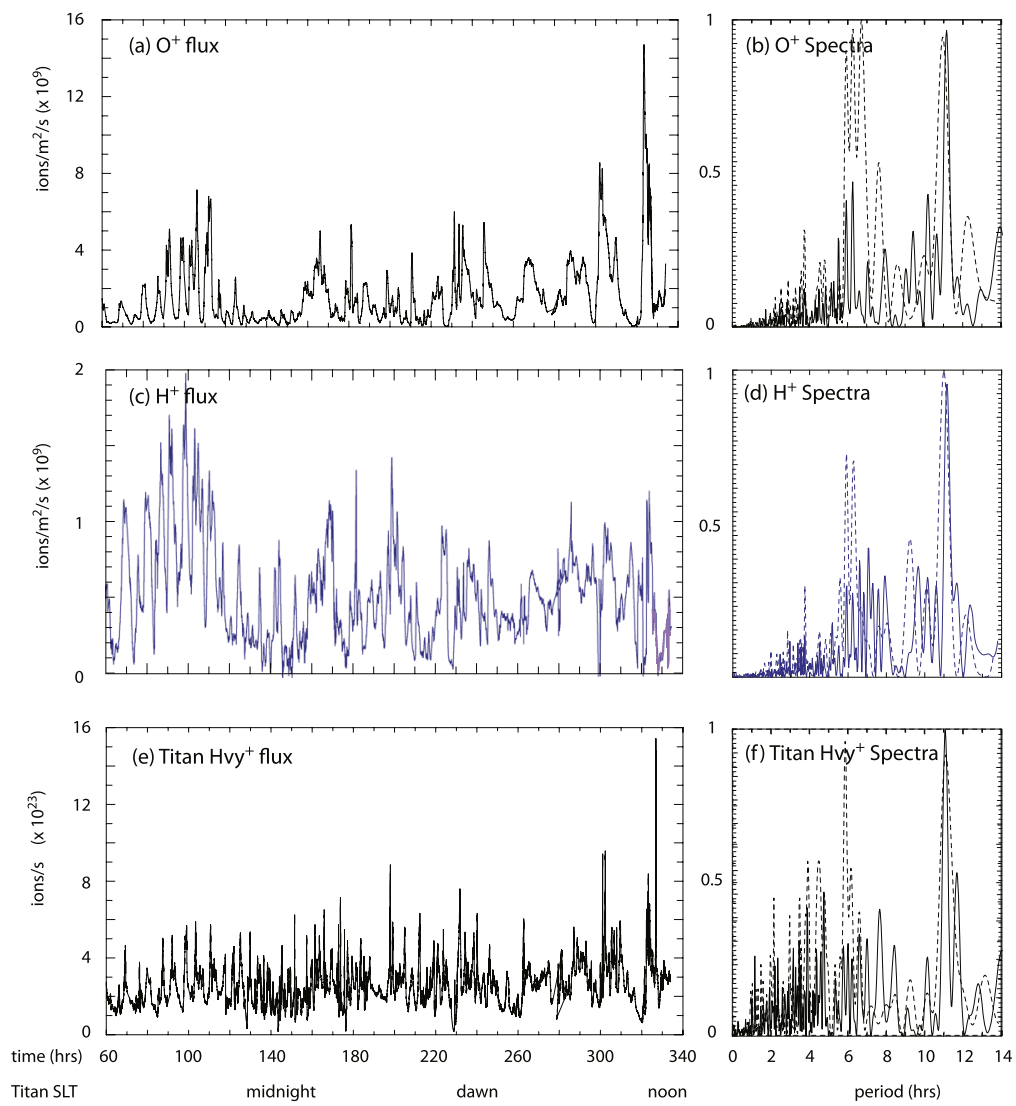

Fig. 27 Left panels show calculated fluxes of $\mathrm{O}^{+}$and $\mathrm{H}^{+}$ions integrated over a $0.4 \mathrm{R}_{\mathrm{S}}$ cube about Titan and the corresponding total flux from Titan. Right panels show the calculated power spectrum derived from the full period (solid line) and between 60-240 hrs (dashed line). Initially the spectrum is dominated by processes at twice the planetary period but, over long timescales, near-planetary periods dominate the fluctuations (from Winglee et al. 2013)

is related to the development of large interchange fingers. As a result, an observer at any fixed azimuthal position will see two distinct spikes in energetic particles moving past them, separated by approximately a planetary period.

Winglee et al. (2013) evaluated Fourier spectra of a variety of physical parameters to determine whether the effects described above could solely produce the observed periods. Figure 27 shows the time history and spectra of the particle fluxes about Titan. The $\mathrm{O}^{+}$ and $\mathrm{H}^{+}$ions originate from the Enceladus torus, while the ions that originate from Titan are designated as $\mathrm{Hvy}^{+}$(heavy ions). It is seen that all three ion species are significantly modulated. For the first 40 hours or so the spectrum is dominated by processes at about twice the planetary period. However after this time the spectrum becomes dominated by processes near the planetary period. This change in characteristic period is consistent with the transient buildup of this resonance process and was missing from the early simulations which considered relatively short periods for the interaction. This evolution towards nearplanetary periods would also be missing from all global simulations which do not include the Titan-related interaction. 
Perturbations in the Inner Magnetosphere

Southern Hemisphere - postnight $R=(8.55,5,-2) R_{S}$
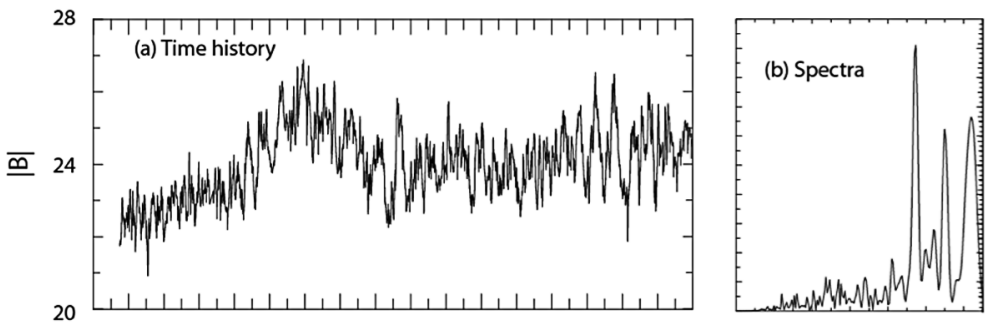

Northern Hemisphere - postnight $R=(8.55,5,2) R_{S}$
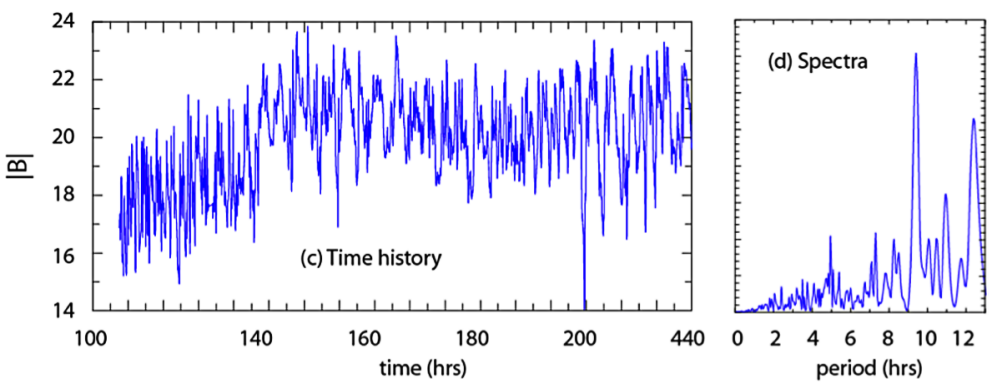

Fig. 28 Comparison of the simulated magnetic perturbations in the northern and southern hemispheres of the Kronian system. The configuration is for southern summer solstice, with Titan being below the center of the magnetodisc. Hence the profiles are not identical, with the northern hemisphere having a slightly shorter period than the southern (from Winglee et al. 2013)

The results in Fig. 27 also show that the particle fluxes moving through the magnetosphere can have substantial temporal variations. Modulations of nearly an order of magnitude can occur and need to be taken into account when trying to fully describe the mass and energy transport in the Kronian magnetosphere. The above model for the periodicities within the Kronian magnetosphere also sheds light on why there are differences between the northern and southern hemisphere periods. This is illustrated in Fig. 28 which shows the time history and Fourier spectra of representative points in the northern and southern hemispheres. The magnetic profiles, while similar, are not identical, and the southern hemisphere generally harbours larger modulations than the northern. This difference occurs because, for the simulated configuration of southern summer solstice, Titan lies below the center of the magnetodisc current sheet. As a result of this difference in proximity, the southern side of the magnetodisc experiences stronger drag and forcing from Titan's induced magnetosphere. This effect produces a slightly longer period for the southern hemisphere relative to that of the northern hemisphere, qualitatively consistent with the Cassini observations cited above. These perturbations are expected to propagate into the magnetosphere where they should be observed simultaneously. However, the simulations have insufficient duration and signal-to-noise ratio to resolve this mixing of the two very close frequencies in the equatorial magnetosphere.

The above results have concentrated mainly on the Kronian magnetosphere due to the wealth of data provided by Cassini. The Jovian magnetosphere, with its stronger magnetic field and stronger influences by the Galilean satellites, is expected to produce an equally rich environment in terms of dynamics and characteristic periods, that has yet to be explored in detail. Because of the stronger magnetic field and the high plasma output from Io (Sect. 1), 
Fig. 29 The ion pickup geometry at Io. The plasma corotating at $74 \mathrm{~km} \mathrm{~s}^{-1}$ meets Io orbiting at $17 \mathrm{~km} \mathrm{~s}^{-1}$. Pickup ions accelerate in a convective electric field corresponding to $57 \mathrm{~km} \mathrm{~s}^{-1}$. Ions follow cycloidal paths in the inertial frame and form rings in velocity space in the plasma rest frame

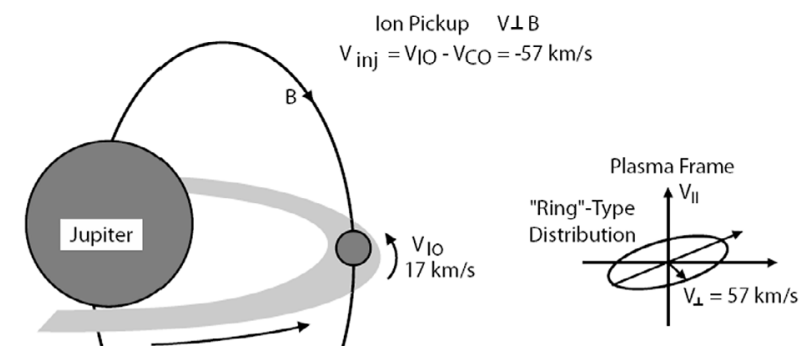

(Adapted from Huddleston et al., 1998)

influence from solar wind forcing on the mass and energy transport within the inner and middle magnetosphere is expected to be less important than for the Kronian system. On the other hand, influences from the Galilean satellites have the potential for being much stronger in terms of moon-magnetosphere interactions. These effects have yet to be explored in detail but, given the physical impact of the effects described above, our understanding of mass and energy transport will change as more data are acquired and improved models developed.

\section{Wave Phenomena in Magnetodisc Regions}

\subsection{Waves in Jupiter's Magnetodisc}

The regions of planetary magnetodiscs where mass loading occurs are rich environments for the generation of low-frequency waves. Ion cyclotron and mirror mode waves have been observed in the magnetospheres of both Jupiter and Saturn. In this section, we will discuss the characteristics of these waves. The waves are similar in character to fluctuations found in other mass loading regions, such as those at Venus, Mars, and comets. Waves are important in this context because they can be used as a diagnostic of the local plasma composition, and they tell us where ion pickup takes place. They also participate in wave-particle energy exchange and play an active role in the interaction between some moons and the giant planetary magnetospheres in which these moons are embedded.

In the Jovian system, ion pick up rates are largest near Io (Sects. 1, 2). It is estimated that of the order $\sim 500-1000 \mathrm{~kg} \mathrm{~s}^{-1}$ of neutral gas associated with the Io source becomes ionized through photoionization, electron impact by a thermal background plasma, electron impact by a superthermal electron population (Delamere and Bagenal 2003), and charge exchange. Once ionized, the material is picked up into the plasma torus - that is, incorporated into the ambient flow of the torus plasma (Hill et al. 1983; Frank and Paterson 1999). Figure 29 shows that Jupiter's magnetic field is approximately perpendicular to the corotational flow of the Io torus plasma, and hence ions are injected into the torus with ring-like velocity distributions which, in turn, are able to provide free energy for wave generation. At the 
orbit of Io, the magnetospheric plasma is rotating around Jupiter with an azimuthal speed of roughly $\sim 74 \mathrm{~km} \mathrm{~s}^{-1}$. Io orbits at $\sim 17 \mathrm{~km} \mathrm{~s}^{-1}$, so the ambient plasma moves at $\sim 57 \mathrm{~km} \mathrm{~s}^{-1}$ relative to Io and its ionosphere. Thus, any newborn ion feels an outward electric field (in the reference frame of Io) corresponding to the product of this $\sim 57 \mathrm{~km} \mathrm{~s}^{-1}$ relative velocity and the $\sim 2000 \mathrm{nT}$ (southward-directed) magnetic field. The newborn ion begins to drift and gyrate about the magnetic field direction, following a cycloidal trajectory in space. In the corotating plasma frame, this motion is circular with a fixed centre and speed of gyration, and the ions thus form a ring-like distribution in velocity space. These ring distributions introduce an effective temperature anisotropy $T_{\perp} / T_{\|}>1$ into the plasma and can therefore lead to the growth of ion cyclotron waves and mirror modes.

Galileo observations showed the existence of ion cyclotron waves in the Io torus during most of the spacecraft's encounters with this moon. The corresponding waves have frequencies near the gyrofrequencies of the ion species $\mathrm{SO}_{2}^{+}, \mathrm{SO}^{+}$and $\mathrm{S}^{+}$. Mirror mode waves were observed only when Galileo crossed Io's wake region. At Europa the mass loading rate representing the pick up of new plasma into the magnetosphere is significantly less than the rate for Io. Galileo observed waves in the Europan wake, with frequencies near the gyrofrequencies of $\mathrm{O}_{2}^{+}$and $\mathrm{Cl}^{+}$ions.

By contrast, Cassini data have revealed the existence of ion cyclotron and mirror mode waves in very extended regions of Saturn's magnetodisc. Thus, at Saturn's magnetosphere, pickup processes occur not only near the moons, but over wider regions in which water group ions are produced at all local times over distance intervals covering several $\mathrm{R}_{\mathrm{S}}$.

\subsubsection{Ion Cyclotron Waves}

Figure 30 shows one example of ion cyclotron waves observed near Io and mirror mode waves observed in the moon's wake. Ion cyclotron waves were observed in the torus during six flybys near Io. The waves had large amplitudes of $\lesssim 100 \mathrm{nT}$, or up to $\sim 5 \%$ of the background field $(\delta B / B \lesssim 0.05)$. These waves also exhibited a left-handed, near-circular polarization, with wave power primarily near the cyclotron frequencies of $\mathrm{SO}_{2}^{+}$and $\mathrm{SO}^{+}$ (Russell and Kivelson 2000; Blanco-Cano et al. 2001a, 2001b; Russell et al. 2003a, 2003b). Wave power became stronger closer to the moon, and the intensity ratio of the $\mathrm{SO}_{2}^{+}$to $\mathrm{SO}^{+}$waves also varied with distance from Io. In some regions, additional cyclotron waves associated with the $\mathrm{S}^{+}$ion were observed.

Figure 31 shows Galileo trajectories for five crossings of the Io wake region. On the first orbit, on December 7, 1995 (flyby I0), Galileo flew $900 \mathrm{~km}$ downstream of Io at closest approach, revealing strong magnetic fluctuations with periods $\sim 2-3 \mathrm{~s}$, corresponding to the gyrofrequencies of $\mathrm{SO}_{2}^{+}$and $\mathrm{SO}^{+}$(Kivelson et al. 1996). The waves were observed inbound and outbound (sinusoidal signatures), extending over distances of $\sim 20 \mathrm{R}_{\mathrm{I}}$ (Io radii) and $\sim 7 \mathrm{R}_{\mathrm{I}}$, respectively. Inbound, the $\mathrm{SO}_{2}^{+}$-related waves had larger amplitudes than those associated with $\mathrm{SO}^{+}$. In contrast, the outbound portion showed intervals with stronger waves near the $\mathrm{SO}^{+}$gyrofrequency, and a region with a burst near the $\mathrm{S}^{+}$gyrofrequency. $\mathrm{SO}_{2}^{+}$ waves reached amplitudes of around $100 \mathrm{nT}$ peak-to-peak, which is consistent with a mass loading rate near Io of about $300 \mathrm{~kg} \mathrm{~s}^{-1}$ (Huddleston et al. 1998). On this orbit, Galileo crossed Io's wake and mirror mode waves were observed at the wake edges (square signatures in Fig. 31; see also Fig. 30, and section below).

On October 11, 1999 (flyby I24) Galileo crossed the upstream nose of the interaction region (Fig. 31a (left panel)), where a weak, short burst of $\mathrm{SO}^{+}$ion cyclotron waves was observed. Later, on Io's downstream side, stronger waves were detected. Power spectra for this flyby (Fig. 32) show two major differences with respect to the waves of orbit I0. Firstly, 
Fig. 30 Galileo measurements of (a) ion cyclotron waves (adapted from Kivelson et al. 1996), and (b) mirror modes (adapted from Russell et al. 1999)
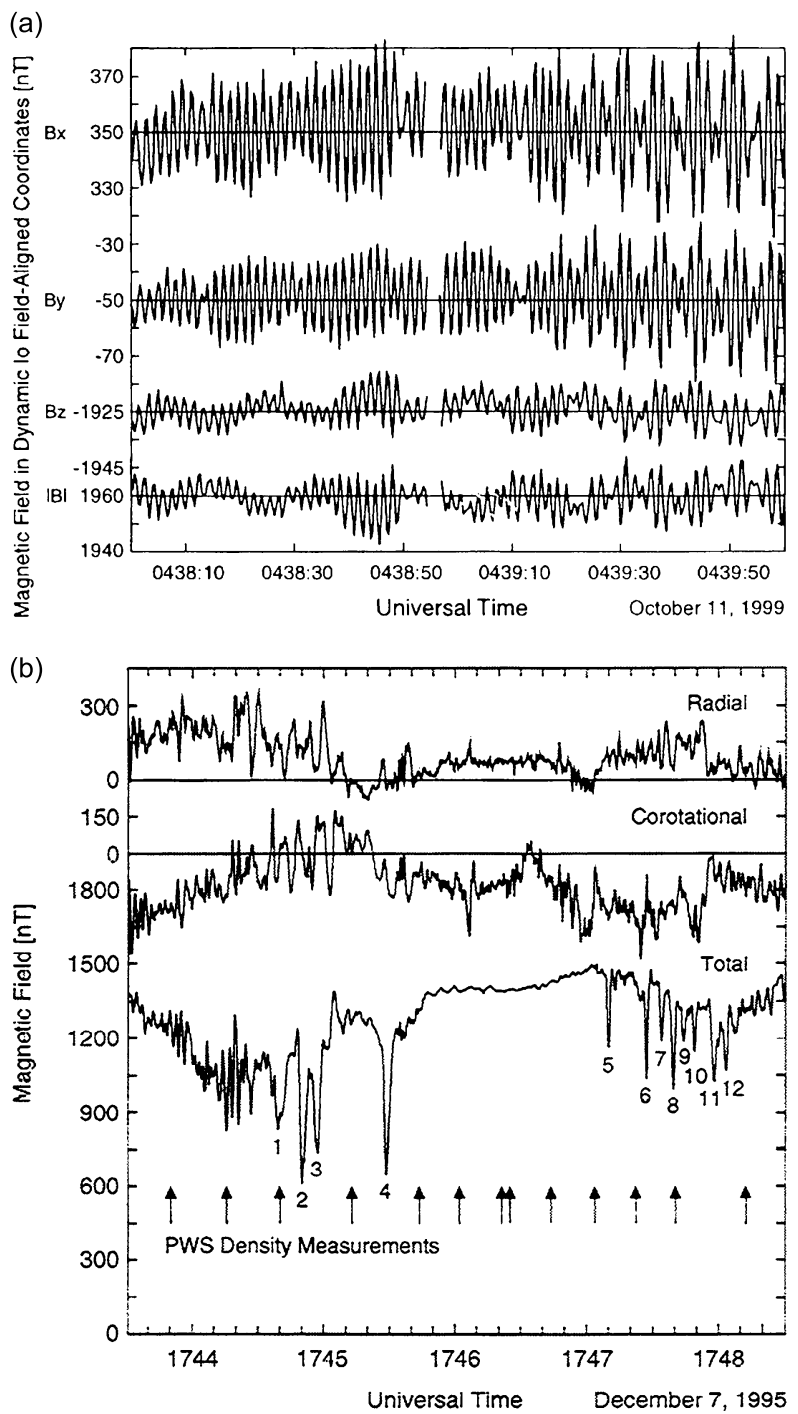

instead of a broad peak, there are two separate narrow peaks at the $\mathrm{SO}_{2}^{+}$and $\mathrm{SO}^{+}$gyrofrequencies (hereafter referred to as $\Omega\left(\mathrm{SO}_{2}^{+}\right)$and $\Omega\left(\mathrm{SO}^{+}\right)$). Secondly, the waves at frequencies near $\Omega\left(\mathrm{SO}^{+}\right)$were stronger and more persistent than those with frequencies closer to $\Omega\left(\mathrm{SO}_{2}^{+}\right)$. In some intervals, a third peak in the spectra appeared near the $\mathrm{S}^{+}$gyrofrequency, denoted $\Omega\left(\mathrm{S}^{+}\right)$. Because there is a background, thermalized component of $\mathrm{S}^{+}$ions in the torus, the presence of waves with frequencies near $\Omega\left(\mathrm{S}^{+}\right)$indicates that there is a strong $\mathrm{S}^{+}$ring distribution which is able to generate the relevant instability for wave growth, and presumably overcome the wave damping due to the thermalized $\mathrm{S}^{+}$population.

On November 26, 1999 (I25), there was only a short interval of Galileo magnetometer data after closest approach to Io. Figure 32c shows two peaks in power, with similar amplitudes at frequencies near $\Omega\left(\mathrm{SO}_{2}^{+}\right)$and $\Omega\left(\mathrm{SO}^{+}\right)$. On February 22, 2000 (I27), Galileo again moved from upstream to downstream (Fig. 31a), and ion cyclotron waves appeared 


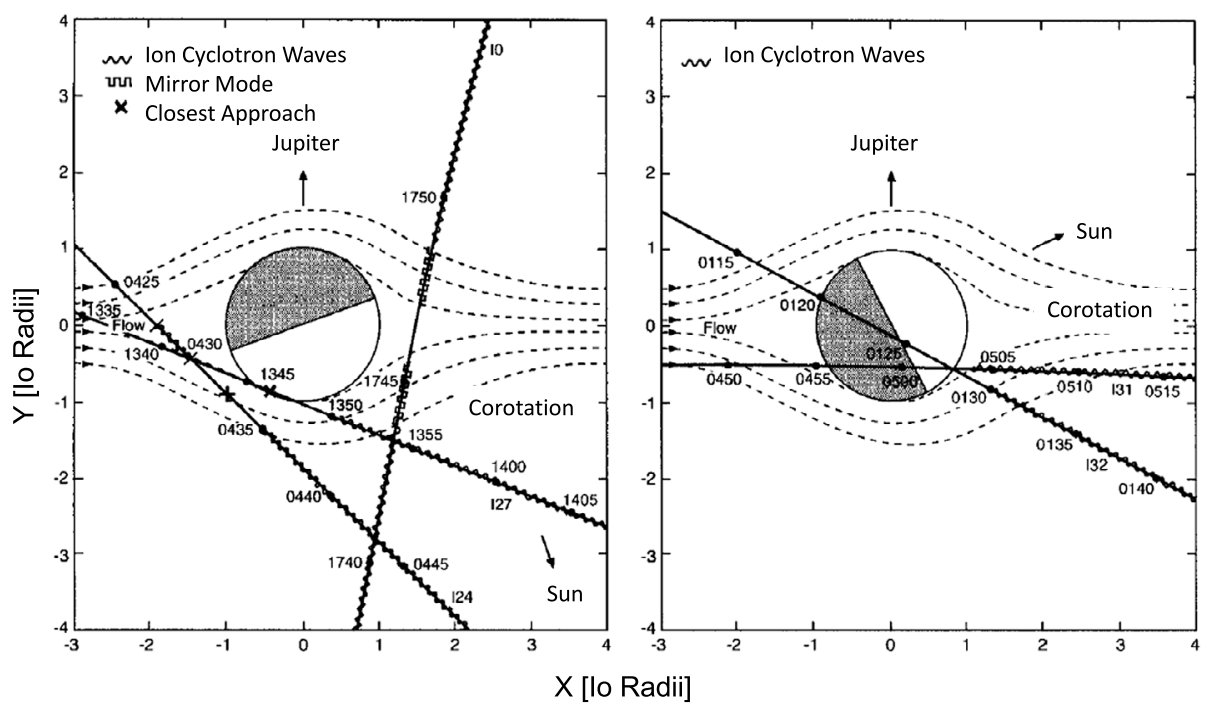

Fig. 31 Galileo trajectories during five encounters with Io. The regions where ion cyclotron waves and mirror modes were observed are indicated by sinusoidal and 'square wave' lines, see legend at top of both panels. The coordinate system keeps the flow velocity to the right and the convective electric field associated with the corotation of the plasma is projected on the Y-direction, outward from Jupiter, according to the instantaneous model field at Io. (Adapted/reprinted from Russell et al. 2003b, Copyright (2003), with permission from Elsevier)
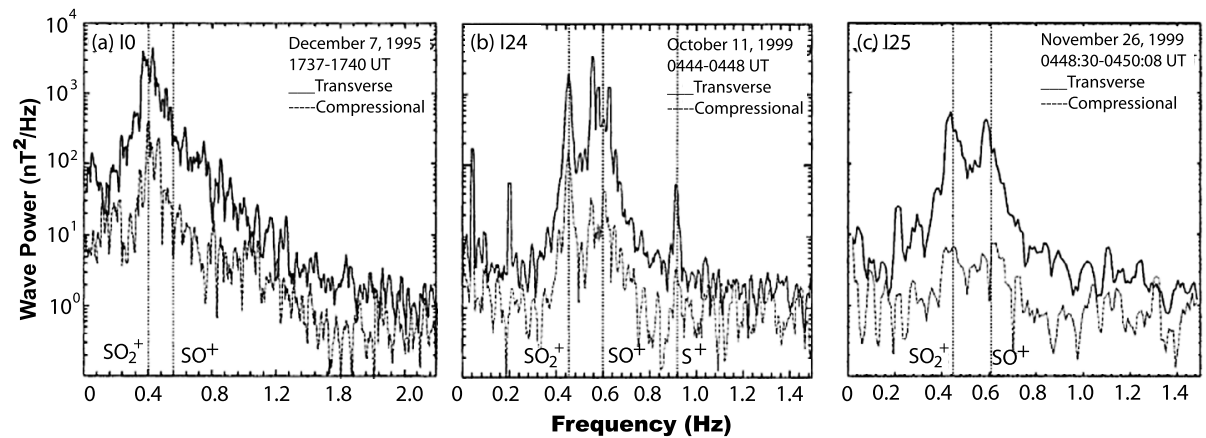

Fig. 32 Transverse and compressional wave power for three intervals with ion cyclotron waves in the Io torus

as Galileo entered the downstream side. Power spectra show peaks near all of the above frequencies $\left(\Omega\left(\mathrm{SO}_{2}^{+}\right), \Omega\left(\mathrm{SO}^{+}\right)\right.$and $\left.\Omega\left(\mathrm{S}^{+}\right)\right)$. In contrast to I24, in some intervals, the amplitude of the peak near $\Omega\left(\mathrm{S}^{+}\right)$is comparable to the $\Omega\left(\mathrm{SO}^{+}\right)$peak.

The last two Io orbits with magnetic field data took place on August 6, 2001 (I31), and October 16, 2001 (I32) (Fig. 31b (right panel)). Both flybys crossed the polar region above or below the moon, so that field lines connected Io and the spacecraft during the relevant time intervals. The I31 orbit was almost parallel to Io's wake, and almost parallel to the corotating flow of the ambient plasma. The field lines through Galileo connected it to the centre of the wake, a region of local minimum in field strength. Wave properties along I31 
are quite different to other passes (Russell et al. 2003a). Power spectra show broad peaks at frequencies below all of the gyrofrequencies $\Omega\left(\mathrm{SO}_{2}^{+}\right), \Omega\left(\mathrm{SO}^{+}\right)$and $\Omega\left(\mathrm{S}^{+}\right)$. The waves exhibit the properties of the ion cyclotron mode in the frequency range $0.3-1.1 \mathrm{~Hz}$. It follows that the waves are generated along field lines where the strength of the magnetic field varies, such that the waves resonate with ions over a broad range of frequencies as they propagate to the higher latitudes where they are observed. For $\mathrm{SO}_{2}^{+}$waves with frequency $0.3 \mathrm{~Hz}, \mathrm{a}$ local field of $1259 \mathrm{nT}$ would be required. Fields this low were indeed encountered on the I0 flyby through the wake.

Finally, orbit I32 had a geometry intermediate between I24 and I27, and wave power peaked near the $\Omega\left(\mathrm{SO}_{2}^{+}\right)$, comparable to the spectra of flyby I0. During orbits $\mathrm{I} 0, \mathrm{I} 24, \mathrm{I} 25$, I27, and I32, the waves observed were transverse, left-handed, and elliptically polarized. Their angle of propagation was variable. In some intervals the waves were almost parallelpropagating (i.e. along the field direction), and almost circularly polarized, while in others they propagate at angles $\lesssim 40^{\circ}$ with respect to the ambient magnetic field (Russell et al. 2001). In contrast, during orbit I31, compressive waves, propagating at large angles to the magnetic field, were also present (Russell et al. 2003a).

Dynamic spectra summarizing wave properties for all flybys are given in Blanco-Cano et al. (2001a) and Russell et al. (2003b). In summary, ion cyclotron waves have generally been observed only downstream from Io, except on the I24 pass when there was a short burst of wave activity upstream of the moon. Their characteristics change along each flyby, and from one orbit to the next. Figure 31 shows that the I0 trajectory crosses both the I 24 and 27 trajectories. However, where they cross, the spectra are quite different on the different passes, suggesting that the physical conditions in the Io atmosphere are quite variable in time. Another interesting point is that ion cyclotron waves can arise far beyond the region in which the flow and the atmosphere should be interacting. The waves are generated by ion ring distributions via the cyclotron instability. They are observed with frequencies mostly close to $\Omega\left(\mathrm{SO}_{2}^{+}\right)$and $\Omega\left(\mathrm{SO}^{+}\right)$. This is because, for other ionic species $\left(\mathrm{O}^{+}, \mathrm{S}^{+}\right.$, $\mathrm{S}_{2}^{++}$) there is a thermalized distribution in the background torus whose presence acts to damp the waves and suppress the instability. On the other hand, because $\mathrm{SO}_{2}^{+}$and $\mathrm{SO}^{+}$ dissociate within tens of minutes, they only exist as ring distributions with no thermalized component.

The existence of $\mathrm{S}^{+}$waves was unexpected because we expect $\mathrm{S}^{+}$background ions to damp any waves generated by $\mathrm{S}^{+}$pickup. Thus, a dense $\mathrm{S}^{+}$ring distribution is indicated to exist in some regions, with a sufficient population for overcoming wave damping. The observed wave variability shows that the Io torus is not uniform, and that ion pickup composition changes with time and spatial location. Since pickup ions originate from Io's atmosphere, observed wave variations suggest that this atmosphere is changing spatially as well as temporally (Russell and Kivelson 2000; Russell et al. 2003b).

\subsubsection{Origin of Ion Cyclotron Waves: Dispersion Analysis and Hybrid Simulations}

It is well known that $\mathrm{SO}_{2}$ and $\mathrm{SO}$ are the main constituents of Io's atmosphere (e.g., Wong and Smyth 2000). The waves associated with the ions of these molecules are generated by ion ring distributions via the cyclotron resonant instability, due to the aforementioned temperature anisotropy in the source plasma. The detection of waves with frequencies near the gyro frequency values $\Omega\left(\mathrm{SO}_{2}^{+}\right)$and $\Omega\left(\mathrm{SO}^{+}\right)$indicates ongoing ionisation and pickup of ions from the atmosphere. However, $\mathrm{SO}_{2}^{+}$and $\mathrm{SO}^{+}$are minor components of the torus plasma (e.g. Frank et al. 1996; Frank and Paterson 2001), since they dissociate within tens 


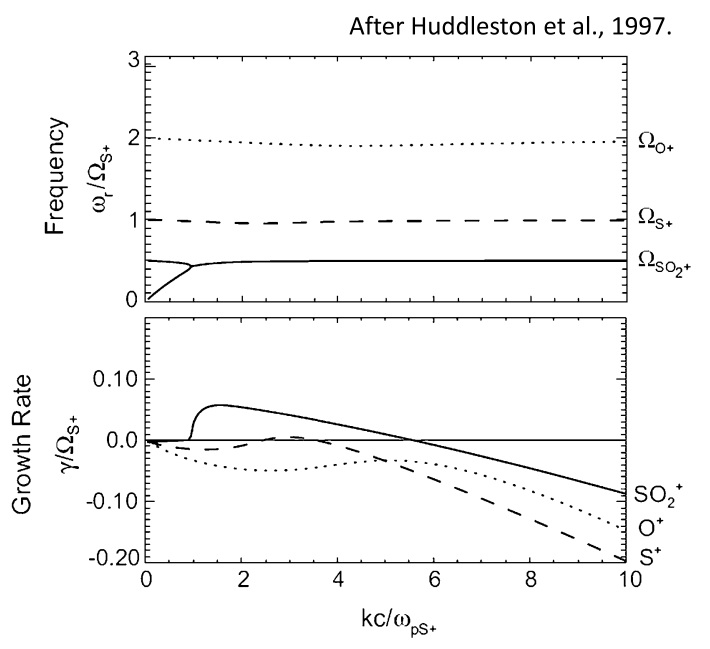

Fig. 33 Wave frequency and growth as a function of wave number for ion cyclotron waves in a multispecies plasma with $\mathrm{O}^{+}, \mathrm{S}^{+}$and $\mathrm{SO}_{2}^{+}$ring distributions, along with $\mathrm{O}^{+}$and $\mathrm{S}^{+}$thermalized background components (after Huddleston et al. 1997). Dispersion curves are for parallel propagating waves

of minutes. The primary constituents of the background plasma torus are $\mathrm{O}^{+}$and $\mathrm{S}^{+}$. However, only weak waves near the $\Omega\left(\mathrm{S}^{+}\right)$were observed. The immediate question that arises is: Why would these minority constituents generate waves while the majority plasma species did not? In a multi-species plasma, multiple ion cyclotron modes are possible, each with a growth rate dependent upon the anisotropy and free energy provided by the specific ion species. To understand the generation of the observed waves, kinetic dispersion analyses for a plasma that resembles the Io torus have been performed. Warnecke et al. (1997) and Huddleston et al. (1997) showed that the presence of the dense $\mathrm{O}^{+} / \mathrm{S}^{+}$thermalized background population damps the waves generated by picked-up $\mathrm{O}^{+}$and $\mathrm{S}^{+}$ions, while, on the other hand, $\mathrm{SO}_{2}^{+}$and $\mathrm{SO}^{+}$waves can grow easily because these ions exist only as ring distributions, without a damping, thermal component.

Figure 33 shows frequency and growth rate for waves propagating parallel to the magnetic field in a plasma which harbours $\mathrm{SO}_{2}^{+}, \mathrm{O}^{+}$and $\mathrm{S}^{+}$ring distributions, along with $\mathrm{O}^{+}$and $\mathrm{S}^{+}$thermalized populations. It is possible to see that the $\mathrm{O}^{+}$and $\mathrm{S}^{+}$wave modes are damped, with negative values of the growth rate parameter $\gamma$, while the $\mathrm{SO}_{2}^{+}$waves remain undamped, and have positive $\gamma$ value for a range of k values. Blanco-Cano et al. (2001a, 2001b) demonstrated that ion cyclotron waves associated with the species $\mathrm{SO}_{2}^{+}, \mathrm{SO}^{+}$and $\mathrm{S}^{+}$can grow simultaneously under certain conditions, and that $\mathrm{S}^{+}$pickup ions can generate waves, provided the density of this component is adequate ( $\gtrsim 10 \%$ of total density) to overcome damping by the thermal background of $\mathrm{S}^{+}$ions. Linear kinetic analysis also shows that, while wave growth at parallel propagation is the most rapid, growth at oblique angles can also be significant (Blanco-Cano et al. 2001b), in agreement with the observations described herein.

More recently, hybrid simulations have been used to study wave generation and nonlinear evolution near Io (Cowee et al. 2006, 2008; Cowee and Gary 2012). These studies have considered continuous ion injection, and are useful for investigating wave-ion energy exchange and the scattering of ion velocity distributions. These works have been successful in reproducing wave spectra observed near Io, and have yielded important insight regarding wave interaction with the various pickup ion populations. 


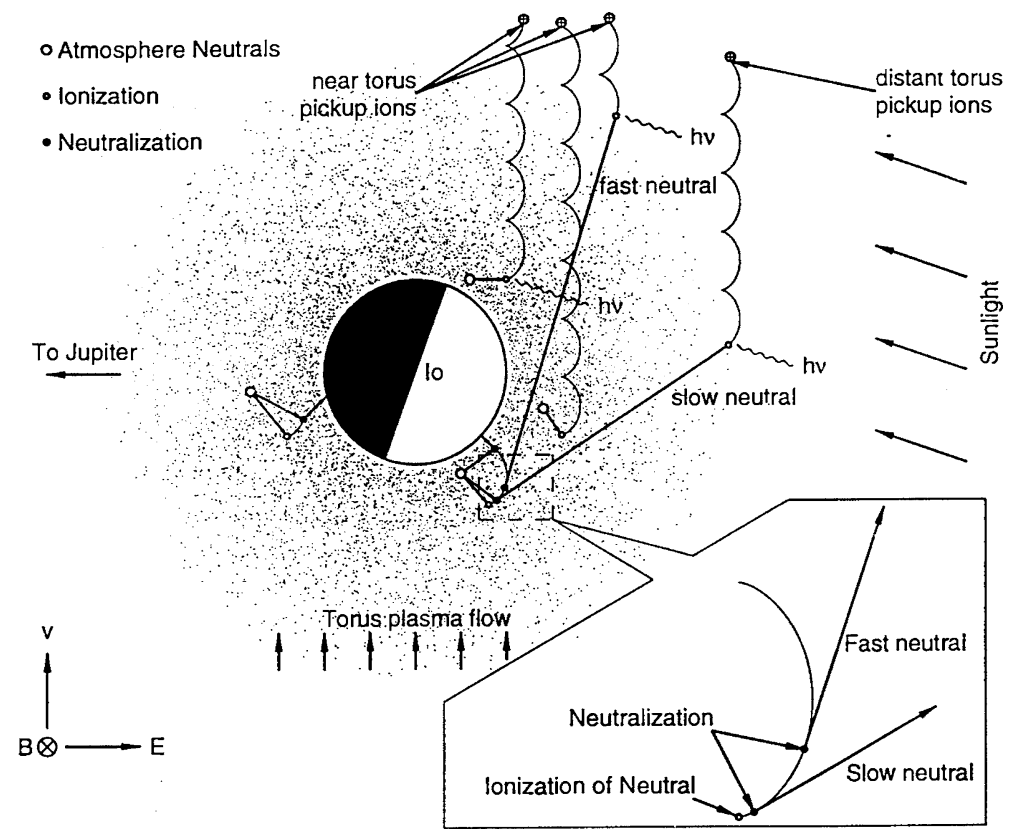

Fig. 34 Mechanism for forming a thin, mass-loading disc that enables the production of pickup ions far to the side of the moon, but not in the upstream direction. Ions are first formed near Io in its moderately dense atmosphere, where charge exchange occurs frequently enough that freshly accelerated ions can be converted to fast neutrals. The fast neutrals then travel for significant distances across the magnetic field before they again become ionized and thus release their free energy in the form of ion cyclotron waves (after Wang et al. 2001)

\subsubsection{Morphology of the Mass Loading Region in the Magnetodisc}

The regions of ion cyclotron wave generation seen by Galileo, and the established absence of this phenomenon in the Voyager data from 1979, $10 \mathrm{R}_{\mathrm{I}}$ beneath Io, constrain the dimensions of the mass loading region where ions are injected into the torus. This region covers a plane extending $\sim 20 \mathrm{R}_{\mathrm{I}}$ in the anti-Jupiter direction, and only $\sim 7 \mathrm{R}_{\mathrm{I}}$ toward Jupiter (see Fig. 31). In the flow direction the region extends downstream at least $\sim 10 \mathrm{R}_{\mathrm{I}}$, but it does not extend upstream of Io. This 'fan-shaped disc' downstream from Io can be produced by the multi-step mechanism proposed by Wang et al. (2001), in which ions are accelerated in the exosphere of Io by the ambient corotational electric field, followed by neutralization and transport across field lines to regions much further from Io, where they can be reionized and picked up into ring distributions, thus generating waves (see Fig. 34). This mechanism limits wave growth to the downstream region, as opposed to growth in a more isotropic source region that would be expected if sputtering were the main mechanism producing Io's neutral torus. Pickup ions produced in this way have their highest fluxes near the wake axis and extend downstream of Io. Observed wave amplitudes are consistent with this distribution of pickup ions, decreasing with distance from the wake axis (Russell et al. 2001).

The fact that ion cyclotron waves appear downstream from Io is also in agreement with recent findings by Dols et al. (2012), who found that Io's atmosphere has longitudinal asymmetries, with a limited radial extension upstream, and a significantly larger scale on the anti-Jovian downstream side. Using a MHD model of the flow and magnetic perturbations 
Fig. 35 Growth rate for ion cyclotron waves and the mirror mode in a plasma with multiple species. The growth of the mirror mode (solid line) benefits from the combined temperature anisotropy of the various ion distributions. Growth rates of the ion cyclotron waves (dashed lines) are smaller (adapted from Huddleston et al. 1999)

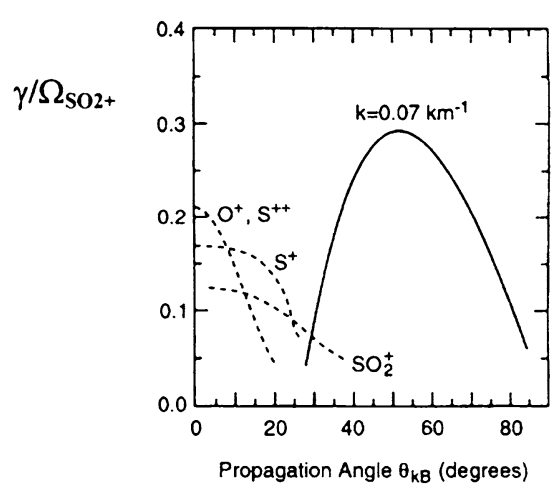

around the moon in conjunction with a multi-species chemistry model, they predicted the formation of a very extended corona of $\mathrm{SO}_{2}$ and $\mathrm{SO}$, mainly downstream from Io.

\subsubsection{Mirror Mode Waves in Io's Wake Region}

As mentioned earlier, during flyby I0, Galileo also observed mirror mode waves on the edges of Io's wake region (see Fig. 30) in addition to the ion cyclotron fluctuations. The waves were highly compressional, with wave normals at large angles $\left(\sim 54^{\circ}-85^{\circ}\right)$ to the local magnetic field. Their properties are discussed in Russell et al. (1999). The mirror mode is typically excited in high-beta plasmas when there is a significant pressure anisotropy (and the condition $P_{\perp} / P_{\|}>1+1 / \beta_{\perp}$ is satisfied), such as that created by pickup ion ring distributions. Maximum growth for the mirror mode occurs at oblique angles, in contrast to the ion cyclotron instability, with maximum growth corresponding to parallel (field-aligned) propagation. The near-stagnant wake region of Io contains a multi-species, anisotropic plasma, a reduced magnetic field, and thus a relatively high plasma $\beta$. Plasma data showed that, at the wake edges, pickup ion densities increased rapidly (Frank et al. 1996), hence providing a large pressure anisotropy. Using kinetic dispersion analysis, Huddleston et al. (1999) showed that in these regions the mirror mode can become dominant.

Figure 35 shows the growth rate of the mirror mode and of $\mathrm{O}^{+}, \mathrm{S}_{2}^{+}, \mathrm{S}^{+}$and $\mathrm{SO}_{2}^{+}$ion cyclotron waves for a plasma which is physically similar to that at the wake edges. While ion cyclotron waves grow due to the anisotropy in the distributions of each species, the mirror mode is fed by the combined anisotropy of all ions present in the plasma and can thus be the dominant plasma perturbation.

\subsubsection{Waves Near Europa}

At Europa, the plasma mass loaded into the magnetosphere per unit time is less than that from the Io source. Interaction of the exosphere with the Jovian magnetosphere produces pickup ion rates of the order of a few $\mathrm{kg} \mathrm{s}^{-1}$ (Saur et al. 1998; Khurana et al. 2002) in contrast to $\sim 1000 \mathrm{~kg} \mathrm{~s}^{-1}$ at Io. Evidence for ion pickup and wave signatures was found on three of eleven passes of Galileo near Europa (Volwerk et al. 2001; Volwerk and Khurana 2010). In contrast to Io, most of the waves were observed in Europa's wake and have been identified as ion cyclotron fluctuations with frequencies near the values $\Omega\left(\mathrm{O}_{2}^{+}\right), \Omega\left(\mathrm{Cl}^{+}\right), \Omega\left(\mathrm{Na}^{+}\right), \Omega\left(\mathrm{Ca}^{+}\right), \Omega\left(\mathrm{K}^{+}\right)$and $\Omega\left(\mathrm{H}_{2} \mathrm{O}^{+}\right)$(using the gyrofrequency nomenclature of previous sections). The waves have both right- and left-hand polarizations. Wave power distributions suggest that the ion pickup rate is larger when Europa is near the 
center of the Jovian current sheet than when the moon is outside it. In a recent work, Volwerk and Khurana (2010) found evidence of $\mathrm{H}_{2} \mathrm{O}^{+}$cyclotron waves upstream of Europa, observed during the Galileo E26 pass.

\subsection{Waves at Saturn}

At Saturn, magnetosphere neutral sources, whether from moons or rings, are weaker than at Jupiter. Nonetheless, Saturn has abundant ion-cyclotron waves, and mirror mode waves have also been observed. In contrast to Jupiter, waves at Saturn appear not only near the moons, but also exist in a extended region covering distances $\sim 4-8 R_{S}$, at all local times, regardless of whether or not the spacecraft was near a moon (Leisner et al. 2005, 2006; Russell et al. 2006).

Ion cyclotron waves were observed at Saturn during the Pioneer 11 and Voyager 1 passes through the system (Smith and Tsurutani 1983; Barbosa 1993). Due to the limited spatial coverage of those flybys, the full radial extent of these waves was not seen until the Cassini spacecraft arrived at Saturn on July 1, 2004 (GMT). In addition to the ion cyclotron waves observed near the fundamental gyrofrequency of pickup ions, there were regions where harmonic ion cyclotron modes were found. Beyond $\sim 6 \mathrm{R}_{\mathrm{S}}$, mirror mode waves and interchanging flux tubes are present and replace the ion cyclotron waves up to regions $\sim 8 R_{S}$ distant from the planet (Russell et al. 2006).

\subsubsection{Ion Cyclotron Wave Properties}

Ion cyclotron waves in Saturn's magnetosphere are generated by water-group ions $\left(\mathrm{O}^{+}\right.$, $\mathrm{OH}^{+}, \mathrm{H}_{2} \mathrm{O}^{+}$). The source of these ions has been identified as Saturn's 'extended neutral cloud', which is primarily sourced by the moon, Enceladus. Enceladus ejects an estimated $10^{27} \mathrm{H}_{2} \mathrm{O}$ molecules/s into the surrounding space through fissures in its surface (e.g., Waite et al. 2006; Tokar et al. 2006). Modelling of these ejected neutrals has shown that they do, indeed, form a neutral cloud which extends over the radial range where the ion cyclotron waves are seen (Johnson et al. 2006). Thus, the source of waves at Saturn is more extended and not strictly limited to the vicinity of a moon (or that moon's torus), as it is at Jupiter.

As in the case of the Jovian magnetosphere, ions in the Kronian system are picked up into ring distributions able to generate waves via the ion cyclotron instability. Such anisotropic $\left(T_{\perp}>T_{\|}\right)$ring-type distributions have been observed near Enceladus (Tokar et al. 2006) and out to radial distance $5 \mathrm{R}_{\mathrm{S}}$ (Tokar et al. 2008). Unsurprisingly, wave properties varied between the Cassini passes, but in general the waves displayed left-handed, near-circular to elliptical polarizations, propagating at angles within $\sim 20^{\circ}$ of the background magnetic field. The wave amplitudes varied, but were typically in the range $0.5-2 \mathrm{nT}$, with peak amplitudes occurring beyond the orbit of Enceladus at around $4-5 \mathrm{R}_{\mathrm{S}}$.

Figure 36a shows power spectra of Cassini observations made at different dates, but at a similar location. It is clear that the largest peak occurred near the gyrofrequencies of water group ions $\left(\Omega\left(\mathrm{O}^{+}\right), \Omega\left(\mathrm{OH}^{+}\right), \Omega\left(\mathrm{H}_{2} \mathrm{O}^{+}\right)\right)$, and that the amplitude of this peak varied for the two dates. A secondary peak appears near the cyclotron frequency of ions with mass $\sim 35 \mathrm{amu}$, which are thought to be $\mathrm{O}_{2}^{+}$. This peak is more prominent on the observation of December 15, 2004. As shown in Fig. 36b, a clear beating structure was seen in the wave time series, indicative of wave generation by these multiple ion species with markedly different masses.

Using many Cassini orbits, Martens et al. (2008) showed that molecular oxygen is a minor constituent of the magnetospheric plasma. Due to the low observed densities of this 
Fig. 36 (a) Power spectrum of ion cyclotron waves at Saturn magnetodisc. These two observations were made when Cassini was at a similar location. (b) Wave series showing three components of the magnetic field. (From Leisner et al. 2006)
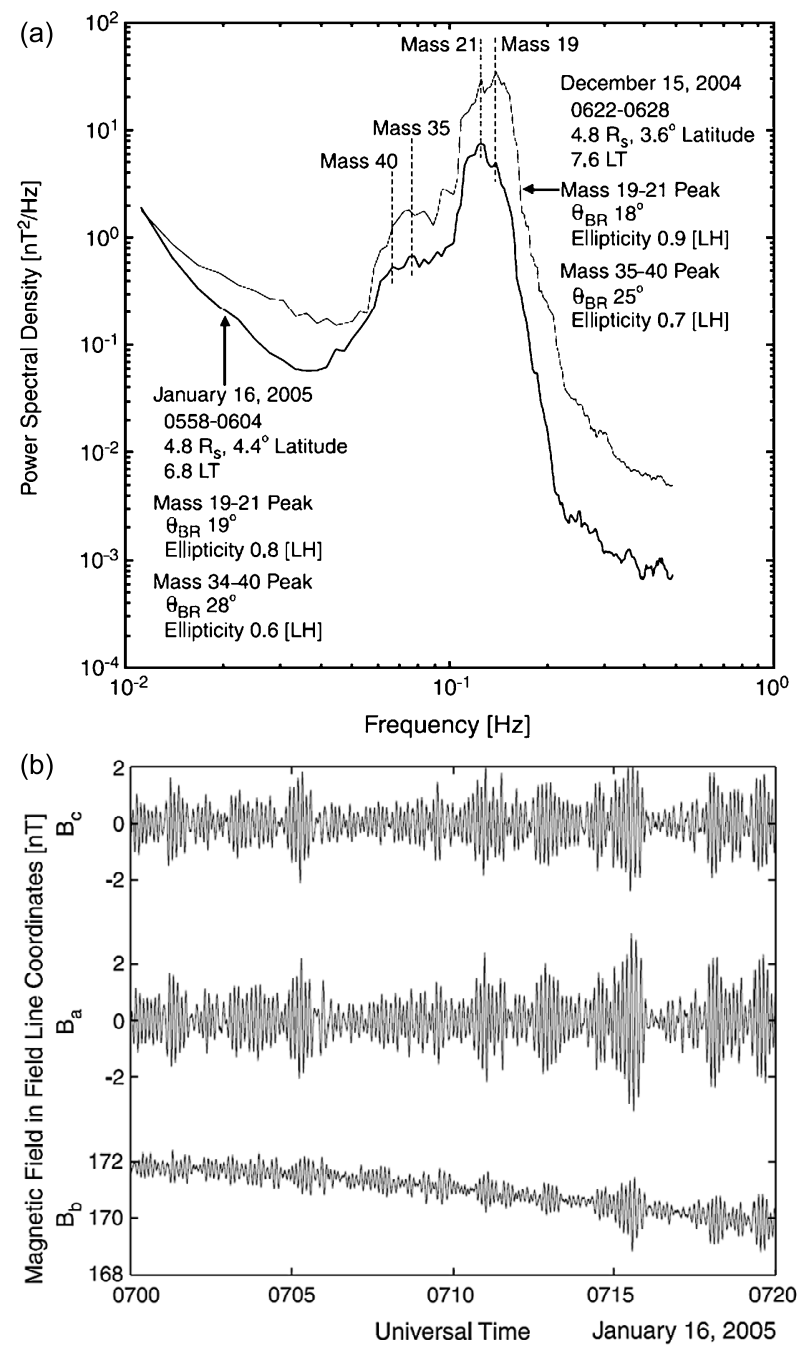

molecule, it is difficult to determine the source, or sources, of the corresponding ions. The two most probable sources are fast neutrals escaping Saturn's main rings and Saturn's dusty E ring. The work by Leisner et al. (2006) supports the conclusion that these ions are a consistent, but minor, plasma species in the magnetosphere. There were a few intervals where the dominant waves had a frequency near the value $\Omega\left(\mathrm{O}_{2}^{+}\right)$(Rodríguez-Martínez et al. 2010). The variation of peak amplitudes indicates that the pickup ion composition changes across the extent of the neutral cloud.

The ion cyclotron waves were observed over a large region of space so the assumption of a homogeneous plasma may not be appropriate. The pickup geometry in the extended neutral cloud region is roughly perpendicular $\left(\alpha \sim 90^{\circ}\right.$, with $\alpha$ the angle between Saturn's magnetic field and $\mathbf{v}$, the velocity of injection of the ions), but the pickup injection velocity also changes with radial distance. Figure 37 shows how the Keplerian and corotational velocities vary with distance from the planet. The pickup velocity may be represented as $V_{\text {pickup }}=$ $V_{K}-V_{c}$, where $V_{K}$ is the Keplerian velocity with which the neutral cloud is orbiting Saturn, 
Fig. 37 Pickup velocities as a function of radial distance. (From Russell et al. 2006)

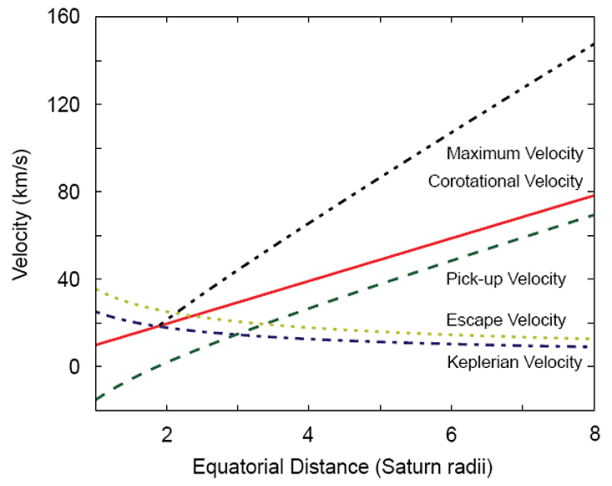

Fig. 38 Wave frequency and growth rate for ion cyclotron waves in Saturn's magnetodisc. Curves are labelled with ion pickup speed (left panels), given in kilometres per second. On right panels, curves correspond to different ring ion density, given in ions per cubic centimetre (from Leisner et al. 2006)
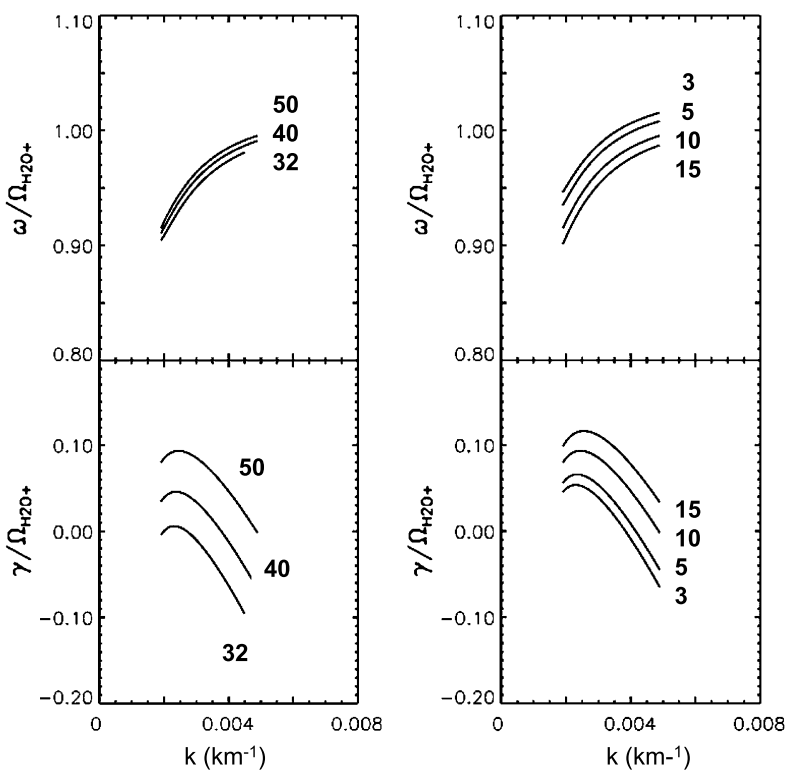

and $V_{c}$ is the local corotation velocity of the ambient plasma (which, of course, changes with radial distance). At a radial distance of $\sim 4 \mathrm{R}_{\mathrm{S}}$, the nominal pickup velocity is $\sim 25 \mathrm{~km} \mathrm{~s}^{-1}$, while at $\sim 8 \mathrm{R}_{\mathrm{S}}$, it is $\sim 70 \mathrm{~km} \mathrm{~s}^{-1}$. Therefore, we may expect that a fixed density of pickup ions at $\sim 4 \mathrm{R}_{\mathrm{S}}$ will be less energetic than those at $\sim 8 \mathrm{R}_{\mathrm{S}}$, and will thus generate loweramplitude waves. There are no ion-cyclotron waves inside $\sim 3.8 \mathrm{R}_{\mathrm{S}}$; ions picked up at these small radial distances will have such small $V_{\text {pickup }}$ values that they will not become unstable to wave generation.

In this context, kinetic dispersion analysis has been very useful to determine how wave growth varies with ring properties. Figure 38 shows the values of wave frequency and growth rate as function of pickup ion injection velocity and density. Ring distributions have more energy to give to the waves as these two values increase, and this is reflected in enhanced values of growth rate $\gamma$. Cassini data also has revealed the presence of weak waves at twice the cyclotron frequency of the water group ions (Rodriguez-Martinez et al. 2008). These waves propagate at angles $\gtrsim 20^{\circ}$ to the background field, and are mainly compressional. Their power is around half that of the fundamental mode. They have been observed on 
multiple Cassini passes and are present only when the background field strength $B_{o}$ exceeds $\sim 150 \mathrm{nT}$.

High-inclination passes by Cassini allowed the study of the vertical structure of the ion cyclotron wave 'belt'. Both the water group and $\mathrm{O}_{2}^{+}$cyclotron waves were found to propagate from the equator up to a vertical distance of $\sim 0.4 \mathrm{R}_{\mathrm{S}}$, and wave amplitude was found to increase off the equator, peaking at a distance of $\sim 0.2 \mathrm{R}_{\mathrm{S}}$ and then rapidly falling off above this limit (Leisner et al. 2011). Why the production region is limited to within $\sim 0.04 R_{S}$ of the magnetic equator, and why the waves rapidly damp out beyond $\sim 0.2 \mathrm{R}_{\mathrm{S}}$ are aspects which have not been explained.

\subsubsection{Mirror Modes}

The ion ring distributions produced by pickup in the E-ring torus are unstable to both ioncyclotron waves and mirror-mode waves. For low plasma beta and moderate temperature anisotropy, the growth rate of the ion-cyclotron waves generally exceeds the growth rate of mirror-mode waves. As described above, ion cyclotron waves have been observed in Saturn's magnetosphere out to $\sim 5 \mathrm{R}_{\mathrm{S}}$. Beyond $\sim 7 \mathrm{R}_{\mathrm{S}}$, mirror mode waves dominate the spectra. As shown in Fig. 39, there is an intermediate region where ion cyclotron waves are dominant, but weaker peaks in the spectra suggest the existence of compressive, mirrormode waves. The simultaneous detection of ion cyclotron and mirror modes is an interesting effect, since linear theory predicts only the mode with the highest growth rate should be observed. At larger distances from Saturn, the mirror mode becomes dominant (see Fig. 39), with strong depths in magnetic field amplitude, and the ion cyclotron waves disappear.

While both instabilities grow from the same pressure anisotropy, the mirror mode dominance at large distances may be due to the fact that the ion cyclotron waves can propagate along the background field out of the wave growth region, while the mirror mode waves remain within it and convect with the bulk motion of the plasma. It is also possible that larger values of plasma beta occur in regions where the mirror mode waves are observed, enhancing the growth of this instability. More work is needed to solve this puzzle.

\subsubsection{The Region Near Titan}

Titan's dense atmosphere is a strong mass-loading source and plasma observations have clearly identified pickup ion populations near this moon (e.g. Hartle et al. 2006; Szego et al. 2005). However, no significant wave power has been identified close to the moon, counter to intuition. In the few examples were some wave growth has been observed near Titan, the waves are mostly left-hand, elliptically polarized (ellipticity $\sim 0.6$ ) and propagate at a large angle $\left(\sim 60^{\circ}\right)$ to the background field. However, the observed wave power is only slightly above the noise level and wave characteristics do not coincide with those of ion cyclotron waves (i.e. near-circular polarization and almost field-aligned propagation).

Linear theory and hybrid simulation results predict that the ion cyclotron ring instability may be unstable at Titan for ideal conditions (i.e. $\alpha=90^{\circ}$ ) but that waves driven by heavy pickup ions (e.g. those with mass-to-charge values $m / q=16$ ) may simply take too long to grow to observable amplitudes before they are convected by the flowing background plasma into the Titan wake (Cowee et al. 2010). Appreciable growth of waves by the lighter ion species (e.g. $m / q \lesssim 2$ ) could be possible on the timescale of plasma convection, however these waves are expected to be strongly damped by the thermalized background plasma. Because Titan is in the outer magnetosphere, the pickup angle and pickup velocity can vary 

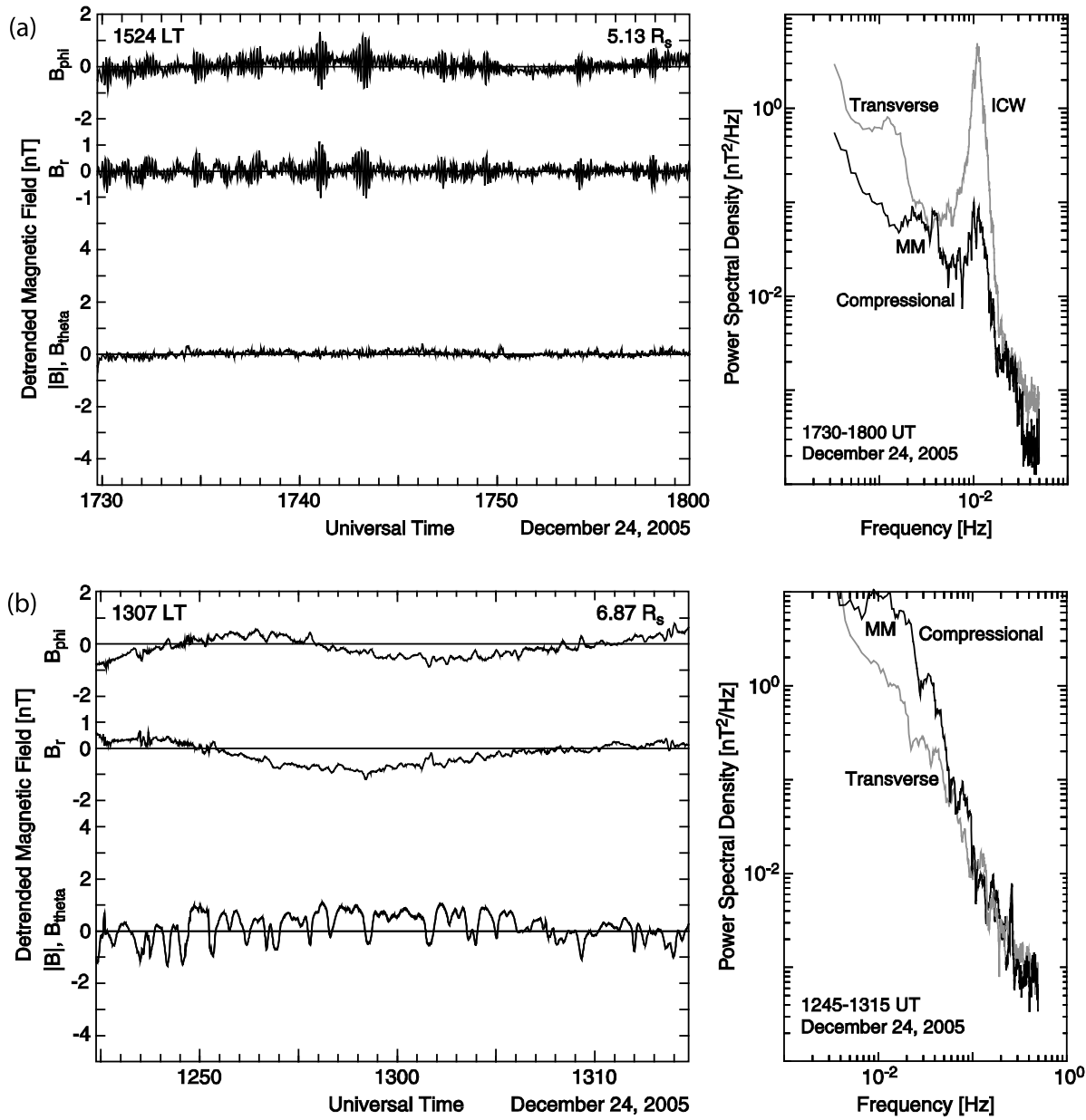

Fig. 39 (a) Ion cyclotron waves and mirror modes: time series and power spectrum in a region where ion cyclotron waves dominate. (b) Time series and power spectrum in a region where the mirror mode is dominant (from Russell et al. 2006)

considerably, especially since the moon may quasi-periodically encounter the centre of the plasma sheet, and it may also, infrequently, emerge into the solar wind. Thus, the pickup velocity may vary between sub-Alfvénic and super-Alfvénic values. Further analysis of the observations is needed, in order to determine under what specific physical conditions ion cyclotron waves can exist near Titan.

\subsection{Conclusions and Future Directions}

Ion cyclotron waves can be generated by pickup ions in Jupiter's and Saturn's magnetodiscs. At Jupiter, the waves appear in a localized region near Io and have frequencies near the gyrofrequencies of the ions $\mathrm{SO}_{2}^{+}, \mathrm{SO}^{+}$and $\mathrm{S}^{+}$. Wave properties are not uniform which indicates that ion composition is not homogeneous and varies due to volcanic activity at Io. Mirror mode waves have been observed only in Io's wake region. Observations made by 
Galileo showed the existence of ion cyclotron waves in Europa's wake. In contrast to the Jovian observations, ion cyclotron waves in the Saturnian magnetodisc appear in larger extensions within the extended neutral cloud. Waves are generated by water group ions picked up from Enceladus and the $\mathrm{E}$ ring. In some regions, $\mathrm{O}_{2}^{+}$cyclotron waves are dominant, indicating variations in pickup ion composition. Ion cyclotron waves extend $\pm 0.3 R_{S}$ out of the equator. Mirror mode waves have also been observed in extended regions of the Saturnian magnetodisc. Pickup ions have been observed near Titan, but no well-defined ion cyclotron waves have been identified there. Future studies concentrating on ion distributions, when available, are needed to understand wave evolution and particle distribution thermalization. Additional simulation studies are also needed to understand the dynamics of waves propagating far from the equatorial disc, the variations in wave propagation properties, and the conditions that favour the mirror mode to be dominant in extended regions of the magnetodisc. Galileo and Cassini data have been very valuable in showing the complexity of these two magnetodiscs. More observations are needed to gain insight regarding the extension of the waves in the region between Io and Jupiter and, in the case of Saturn, to have a better understanding of the wave belt which lies out of the equator.

Acknowledgements NA wishes to acknowledge the support of the ISSI and the STFC UCL Astrophysics Consolidated Grant ST/J001511/1; he also wishes to acknowledge useful discussions with staff at the Japanese Aerospace Exploration Agency (JAXA) during a Visiting Professorship. PB was supported by NASA Grants NNX10AF17G and NNX12AG81G. Nicolas André wishes to acknowledge the support of CNES for Cassini data analysis, and CDPP for the use of the AMDA (Automated Multi-Dataset Analysis) tool available at URL http://cdpp.cesr.fr. XBC acknowledges support of the ISSI and UNAM-DGAPAPAPIIT grants IN110511 and IN105014. The authors acknowledge the support of EUROPLANET RI project (Grant agreement no.: 228319) funded by EU; and also the support of the International Space Science Institute (Bern). The Editor thanks the work of two anonymous referees.

Open Access This article is distributed under the terms of the Creative Commons Attribution License which permits any use, distribution, and reproduction in any medium, provided the original author(s) and the source are credited.

\section{References}

N. Achilleos, S. Miller, R. Prangé, G. Millward, M.K. Dougherty, A dynamical model of Jupiter's auroral electrojet. New J. Phys. 3, 3 (2001). doi:10.1088/1367-2630/3/1/303

N. Achilleos, P. Guio, C.S. Arridge, A model of force balance in Saturn's magnetodisc. Mon. Not. R. Astron. Soc. 401, 2349-2371 (2010). doi:10.1111/j.1365-2966.2009.15865.x

N. André, K.M. Ferrière, Low-frequency waves and instabilities in stratified, gyrotropic, multicomponent plasmas: Theory and application to plasma transport in the Io torus. J. Geophys. Res. 109, 12225 (2004). doi:10.1029/2004JA010599

N. André, K.M. Ferrière, Comments on Vasyliunas' and Pontius' studies of the effects of the planetary ionosphere and of the Coriolis force on the interchange instability. J. Geophys. Res. 112, 10203 (2007). doi:10.1029/2006JA011732

N. André, M.K. Dougherty, C.T. Russell, J.S. Leisner, K.K. Khurana, Dynamics of the Saturnian inner magnetosphere: First inferences from the Cassini magnetometers about small-scale plasma transport in the magnetosphere. Geophys. Res. Lett. 32, 14 (2005). doi:10.1029/2005GL022643

N. André, A.M. Persoon, J. Goldstein, J.L. Burch, P. Louarn, G.R. Lewis, A.M. Rymer, A.J. Coates, W.S. Kurth, E.C. Sittler, M.F. Thomsen, F.J. Crary, M.K. Dougherty, D.A. Gurnett, D.T. Young, Magnetic signatures of plasma-depleted flux tubes in the Saturnian inner magnetosphere. Geophys. Res. Lett. 34, 14108 (2007). doi:10.1029/2007GL030374

D.J. Andrews, S.W.H. Cowley, M.K. Dougherty, L. Lamy, G. Provan, D.J. Southwood, Planetary period oscillations in Saturn's magnetosphere: Evolution of magnetic oscillation properties from southern summer to post-equinox. J. Geophys. Res. 117, 4224 (2012). doi:10.1029/2011JA017444

C.S. Arridge, N. André, K.K. Khurana, C.T. Russell, S.W.H. Cowley, G. Provan, D.J. Andrews, C.M. Jackman, A.J. Coates, E.C. Sittler, M.K. Dougherty, D.T. Young, Periodic motion of Saturn's nightside plasma sheet. J. Geophys. Res. 116, 11205 (2011). doi:10.1029/2011JA016827 
F. Bagenal, Ionization source near Io from Galileo wake data. Geophys. Res. Lett. 24, 2111 (1997). doi:10.1029/97GL02052

F. Bagenal, P.A. Delamere, Flow of mass and energy in the magnetospheres of Jupiter and Saturn. J. Geophys. Res. 116, 5209 (2011). doi:10.1029/2010JA016294

D.D. Barbosa, Theory and observations of electromagnetic ion cyclotron waves in Saturn's inner magnetosphere. J. Geophys. Res. 98, 9345-9350 (1993). doi:10.1029/93JA00476

M. Blanc, S. Bolton, J. Bradley, M. Burton, T.E. Cravens, I. Dandouras, M.K. Dougherty, M.C. Festou, J. Feynman, R.E. Johnson, T.G. Gombosi, W.S. Kurth, P.C. Liewer, B.H. Mauk, S. Maurice, D. Mitchell, F.M. Neubauer, J.D. Richardson, D.E. Shemansky, E.C. Sittler, B.T. Tsurutani, P. Zarka, L.W. Esposito, E. Grün, D.A. Gurnett, A.J. Kliore, S.M. Krimigis, D. Southwood, J.H. Waite, D.T. Young, Magnetospheric and plasma science with Cassini-Huygens. Space Sci. Rev. 104, 253-346 (2002). doi:10.1023/A:1023605110711

X. Blanco-Cano, C.T. Russell, R.J. Strangeway, The Io mass-loading disk: Wave dispersion analysis. J. Geophys. Res. 106, 26261-26276 (2001a). doi:10.1029/2001JA900090

X. Blanco-Cano, C.T. Russell, R.J. Strangeway, M.G. Kivelson, K.K. Khurana, Galileo observations of ion cyclotron waves in the Io torus. Adv. Space Res. 28, 1469-1474 (2001b). doi:10.1016/S02731177(01)00548-8

X. Blanco-Cano, C.T. Russell, D.E. Huddleston, R.J. Strangeway, Ion cyclotron waves near Io. Planet. Space Sci. 49, 1125-1136 (2001c). doi:10.1016/S0032-0633(01)00020-4

P.C. Brandt, C.P. Paranicas, J.F. Carbary, D.G. Mitchell, B.H. Mauk, S.M. Krimigis, Understanding the global evolution of Saturn's ring current. Geophys. Res. Lett. 35, 17101 (2008). doi:10.1029/2008GL034969

P.C. Brandt, D.G. Mitchell, D.A. Gurnett, A.M. Persoon, N.A. Tsyganenko, Saturn's periodic magnetosphere: the relation between periodic hot plasma injections, a rotating partial ring current, global magnetic field distortions, plasmapause motion, and radio emissions, in EGU General Assembly Conference Abstracts, ed. by A. Abbasi, N. Giesen. EGU General Assembly Conference Abstracts, vol. 14 (2012), p. 12906

S.H. Brecht, J.G. Luhmann, D.J. Larson, Simulation of the Saturnian magnetospheric interaction with Titan. J. Geophys. Res. 105, 13119-13130 (2000). doi:10.1029/1999JA900490

N. Brice, T.R. McDonough, Jupiter's radiation belts. Icarus 18, 206-219 (1973). doi:10.1016/00191035(73)90204-2

R.A. Brown, The Jupiter hot plasma torus: Observed electron temperature and energy flows. Astrophys. J. 244, 1072 (1981)

E.J. Bunce, S.W.H. Cowley, D.M. Wright, A.J. Coates, M.K. Dougherty, N. Krupp, W.S. Kurth, A.M. Rymer, In situ observations of a solar wind compression-induced hot plasma injection in Saturn's tail. Geophys. Res. Lett. 32, 20 (2005). doi:10.1029/2005GL022888

E.J. Bunce, S.W.H. Cowley, I.I. Alexeev, C.S. Arridge, M.K. Dougherty, J.D. Nichols, C.T. Russell, Cassini observations of the variation of Saturn's ring current parameters with system size. J. Geophys. Res. 112(A11), 10202 (2007). doi:10.1029/2007JA012275

E.J. Bunce, C.S. Arridge, J.T. Clarke, A.J. Coates, S.W.H. Cowley, M.K. Dougherty, J.-C. GéRard, D. Grodent, K.C. Hansen, J.D. Nichols, D.J. Southwood, D.L. Talboys, Origin of Saturn's aurora: Simultaneous observations by Cassini and the Hubble Space Telescope. J. Geophys. Res. 113, 9209 (2008). doi:10.1029/2008JA013257

J.L. Burch, J. Goldstein, T.W. Hill, D.T. Young, F.J. Crary, A.J. Coates, N. André, W.S. Kurth, E.C. Sittler, Properties of local plasma injections in Saturn's magnetosphere. Geophys. Res. Lett. 32, 14 (2005). doi:10.1029/2005GL022611

J.L. Burch, J. Goldstein, W.S. Lewis, D.T. Young, A.J. Coates, M.K. Dougherty, N. André, Tethys and Dione as sources of outward-flowing plasma in Saturn's magnetosphere. Nature 447, 833-835 (2007). doi:10.1038/nature05906

J.L. Burch, A.D. DeJong, J. Goldstein, D.T. Young, Periodicity in Saturn's magnetosphere: Plasma cam. Geophys. Res. Lett. 36, 14203 (2009). doi:10.1029/2009GL039043

M.H. Burger, Io's neutral clouds: From the atmosphere to the plasma torus, PhD thesis, University of Colorado at Boulder, 2003

J.F. Carbary, D.G. Mitchell, Periodicities in Saturn's magnetosphere. Rev. Geophys. 51, 1-30 (2013). doi:10.1002/rog.20006

J.F. Carbary, D.G. Mitchell, S.M. Krimigis, D.C. Hamilton, N. Krupp, Charged particle periodicities in Saturn's outer magnetosphere. J. Geophys. Res. 112, 6246 (2007). doi:10.1029/2007JA012351

J.F. Carbary, D.G. Mitchell, P. Brandt, C. Paranicas, S.M. Krimigis, ENA periodicities at Saturn. Geophys. Res. Lett. 35, 7102 (2008a). doi:10.1029/2008GL033230

J.F. Carbary, D.G. Mitchell, P. Brandt, E.C. Roelof, S.M. Krimigis, Track analysis of energetic neutral atom blobs at Saturn. J. Geophys. Res. 113, 1209 (2008b). doi:10.1029/2007JA012708

T.A. Cassidy, R.E. Johnson, Collisional spreading of Enceladus's neutral cloud. Icarus 209, 696-703 (2010). doi:10.1016/j.icarus.2010.04.010 
G. Caudal, A self-consistent model of Jupiter's magnetodisc including the effects of centrifugal force and pressure. J. Geophys. Res. 91, 4201-4221 (1986). doi:10.1029/JA091iA04p04201

C.X. Chen, Numerical simulation of the Io-torus-driven radial plasma transport. J. Geophys. Res. 108, 1376 (2003). doi:10.1029/2002JA009460

Y. Chen, T.W. Hill, Statistical analysis of injection/dispersion events in Saturn's inner magnetosphere. J. Geophys. Res. 113, 7215 (2008). doi:10.1029/2008JA013166

Y. Chen, T.W. Hill, A.M. Rymer, R.J. Wilson, Rate of radial transport of plasma in Saturn's inner magnetosphere. J. Geophys. Res. 115, 10211 (2010). doi:10.1029/2010JA015412

A.F. Cheng, Magnetospheric interchange instability. J. Geophys. Res. 90, 9900-9904 (1985). doi:10.1029/ JA090iA10p09900

J.T. Clarke, L. Ben Jaffel, J.-C. Gérard, Hubble Space Telescope imaging of Jupiter's UV aurora during the Galileo orbiter mission. J. Geophys. Res. 103, 20217-20236 (1998). doi:10.1029/98JE01130

K.E. Clarke, D.J. Andrews, C.S. Arridge, A.J. Coates, S.W.H. Cowley, Magnetopause oscillations near the planetary period at Saturn: Occurrence, phase, and amplitude. J. Geophys. Res. 115, 8209 (2010). doi:10.1029/2009JA014745

M.R. Combi, K. Kabin, T.I. Gombosi, D.L. DeZeeuw, Io's plasma environment during the Galileo flyby: Global three-dimensional MHD modeling with adaptive mesh refinement. J. Geophys. Res. 103, 9071 (1998)

M.M. Cowee, S.P. Gary, Electromagnetic ion cyclotron wave generation by planetary pickup ions: Onedimensional hybrid simulations at sub-Alfvénic pickup velocities. J. Geophys. Res. 117, 6215 (2012). doi:10.1029/2012JA017568

M.M. Cowee, R.J. Strangeway, C.T. Russell, D. Winske, One-dimensional hybrid simulations of planetary ion pickup: Techniques and verification. J. Geophys. Res. 111, 12213 (2006). doi:10.1029/2006JA011996

M.M. Cowee, C.T. Russell, R.J. Strangeway, One-dimensional hybrid simulations of planetary ion pickup: Effects of variable plasma and pickup conditions. J. Geophys. Res. 113, 8220 (2008). doi:10.1029/ 2008JA013066

M.M. Cowee, S.P. Gary, H.Y. Wei, R.L. Tokar, C.T. Russell, An explanation for the lack of ion cyclotron wave generation by pickup ions at Titan: 1-D hybrid simulation results. J. Geophys. Res. 115, 10224 (2010). doi:10.1029/2010JA015769

S.W.H. Cowley, E.J. Bunce, Origin of the main auroral oval in Jupiter's coupled magnetosphere-ionosphere system. Planet. Space Sci. 49, 1067-1088 (2001). doi:10.1016/S0032-0633(00)00167-7

S.W.H. Cowley, J.D. Nichols, D.J. Andrews, Modulation of Jupiter's plasma flow, polar currents, and auroral precipitation by solar wind-induced compressions and expansions of the magnetosphere: a simple theoretical model. Ann. Geophys. 25, 1433-1463 (2007). doi:10.5194/angeo-25-1433-2007

F.J. Crary, F. Bagenal, Ion cyclotron waves, pickup ions, and Io's neutral exosphere. J. Geophys. Res. 105, 25379-27066 (2000). doi:10.1029/2000JA000055

F.J. Crary, F. Bagenal, J.A. Ansher, D.A. Gurnett, W.S. Kurth, Anisotropy and proton density in the Io plasma torus derived from whistler wave dispersion. J. Geophys. Res. 101, 2699-2706 (1996). doi:10.1029/ 95JA02212

P.A. Delamere, F. Bagenal, Modeling variability of plasma conditions in the Io torus. J. Geophys. Res. 108, $1276(2003)$

P.A. Delamere, F. Bagenal, Longitudinal plasma density variations at Saturn caused by hot electrons. Geophys. Res. Lett. 35, 3107 (2008). doi:10.1029/2007GL031095

P.A. Delamere, F. Bagenal, Jupiter and Saturn: Colossal comets? (2013 in preparation)

P.A. Delamere, F. Bagenal, V. Dols, L.C. Ray, Saturn's neutral torus versus Jupiter's plasma torus. Geophys. Res. Lett. 34, 9105 (2007). doi:10.1029/2007GL029437

M.D. Desch, M.L. Kaiser, Voyager measurement of the rotation period of Saturn's magnetic field. Geophys. Res. Lett. 8, 253-256 (1981). doi:10.1029/GL008i003p00253

V. Dols, P.A. Delamere, F. Bagenal, A multispecies chemistry model of Io's local interaction with the Plasma Torus. J. Geophys. Res. 113, 9208 (2008). doi:10.1029/2007JA012805

V. Dols, P.A. Delamere, F. Bagenal, W.S. Kurth, W.R. Paterson, Asymmetry of Io's outer atmosphere: Constraints from five Galileo flybys. J. Geophys. Res., Planets 117, 10010 (2012). doi:10.1029/ 2012JE004076

S.A. Espinosa, M.K. Dougherty, Periodic perturbations in Saturn's magnetic field. Geophys. Res. Lett. 27, 2785-2788 (2000). doi:10.1029/2000GL000048

A.J. Farmer, Saturn in hot water: Viscous evolution of the Enceladus torus. Icarus 202, 280-286 (2009). doi:10.1016/j.icarus.2009.02.031

K.M. Ferrière, N. André, A mixed magnetohydrodynamic-kinetic theory of low-frequency waves and instabilities in stratified, gyrotropic, two-component plasmas. J. Geophys. Res. 108, 1308 (2003). doi:10.1029/ 2003JA009883 
K.M. Ferrière, C. Zimmer, M. Blanc, Magnetohydrodynamic waves and gravitational/centrifugal instability in rotating systems. J. Geophys. Res. 104, 17335-17356 (1999). doi:10.1029/1999JA900167

B.L. Fleshman, The roles of dissociation and velocity-dependent charge exchange in Saturn's extended neutral clouds, in Magnetospheres of the Outer Planets (2011)

B.L. Fleshman, P.A. Delamere, F. Bagenal, A sensitivity study of the Enceladus torus. J. Geophys. Res., Planets 115, 4007 (2010a). doi:10.1029/2009JE003372

B.L. Fleshman, P.A. Delamere, F. Bagenal, Modeling the Enceladus plume-plasma interaction. Geophys. Res. Lett. 37, 3202 (2010b). doi:10.1029/2009GL041613

B.L. Fleshman, P.A. Delamere, F. Bagenal, T. Cassidy, The roles of charge exchange and dissociation in spreading Saturn's neutral clouds. J. Geophys. Res., Planets 117, 5007 (2012). doi:10.1029/ 2011JE003996

L.A. Frank, W.R. Paterson, Intense electron beams observed at Io with the Galileo spacecraft. J. Geophys. Res. 104, 28657 (1999)

L.A. Frank, W.R. Paterson, Production of hydrogen ions at Io. J. Geophys. Res. 104, 10345-10354 (1999). doi:10.1029/1999JA900052

L.A. Frank, W.R. Paterson, Observations of plasmas in the Io torus with the Galileo spacecraft. J. Geophys. Res. 105, 16017-16034 (2000). doi:10.1029/1999JA000250

L.A. Frank, W.R. Paterson, Passage through lo's ionospheric plasmas by the Galileo spacecraft. J. Geophys. Res. 106(A11), 26209-26224 (2001). doi:10.1029/2000JA002503

L.A. Frank, W.R. Paterson, K.L. Ackerson, V.M. Vasyliunas, F.V. Coroniti, S.J. Bolton, Plasma observations at Io with the Galileo spacecraft. Science 274(5286), 394-395 (1996). doi:10.1126/science. 274.5286.394

K. Fukazawa, T. Ogino, R.J. Walker, Vortex-associated reconnection for northward IMF in the Kronian magnetosphere. Geophys. Res. Lett. 34, 23201 (2007)

K. Fukazawa, T. Ogino, R.J. Walker, A simulation study of dynamics in the distant Jovian magnetotail. J. Geophys. Res. 115, 9219 (2010). doi:10.1029/2009JA015228

G. Giampieri, M.K. Dougherty, E.J. Smith, C.T. Russell, A regular period for Saturn's magnetic field that may track its internal rotation. Nature 441, 62-64 (2006). doi:10.1038/nature04750

J.A. Gledhill, Magnetosphere of Jupiter. Nature 214, 155 (1967). doi:10.1038/214155a0

T. Gold, Motions in the magnetosphere of the earth. J. Geophys. Res. 64, 1219-1224 (1959)

P. Goldreich, A.J. Farmer, Spontaneous axisymmetry breaking of the external magnetic field at Saturn. J. Geophys. Res. 112, 5225 (2007). doi:10.1029/2006JA012163

D.A. Gurnett, W.S. Kurth, G.B. Hospodarsky, A.M. Persoon, T.F. Averkamp, B. Cecconi, A. Lecacheux, P. Zarka, P. Canu, N. Cornilleau-Wehrlin, P. Galopeau, A. Roux, C. Harvey, P. Louarn, R. Bostrom, G. Gustafsson, J.-E. Wahlund, M.D. Desch, W.M. Farrell, M.L. Kaiser, K. Goetz, P.J. Kellogg, G. Fischer, H.-P. Ladreiter, H. Rucker, H. Alleyne, A. Pedersen, Radio and plasma wave observations at Saturn from Cassini's approach and first orbit. Science 307, 1255-1259 (2005). doi:10.1126/science.1105356

D.A. Gurnett, A.M. Persoon, W.S. Kurth, J.B. Groene, T.F. Averkamp, M.K. Dougherty, D.J. Southwood, The variable rotation period of the inner region of Saturn's plasma disk. Science 316, 442 (2007). doi: $10.1126 /$ science. 1138562

D.A. Gurnett, A.M. Persoon, J.B. Groene, A.J. Kopf, G.B. Hospodarsky, W.S. Kurth, A north-south difference in the rotation rate of auroral hiss at Saturn: Comparison to Saturn's kilometric radio emission. Geophys. Res. Lett. 36, 21108 (2009). doi:10.1029/2009GL040774

K.C. Hansen, A.J. Ridley, G.B. Hospodarsky, N. Achilleos, M.K. Dougherty, T.I. Gombosi, G. Tóth, Global MHD simulations of Saturn's magnetosphere at the time of Cassini approach. Geophys. Res. Lett. 32, 20 (2005). doi:10.1029/2005GL022835

R.E. Hartle, E.C. Sittler, F.M. Neubauer, R.E. Johnson, H.T. Smith, F. Crary, D.J. McComas, D.T. Young, A.J. Coates, D. Simpson, S. Bolton, D. Reisenfeld, K. Szego, J.J. Berthelier, A. Rymer, J. Vilppola, J.T. Steinberg, N. Andre, Initial interpretation of Titan plasma interaction as observed by the Cassini plasma spectrometer: Comparisons with Voyager 1. Planet. Space Sci. 54, 1211-1224 (2006). doi:10.1016/j.pss.2006.05.029

S.L.G. Hess, P. Delamere, V. Dols, B. Bonfond, D. Swift, Power transmission and particle acceleration along the Io flux tube. J. Geophys. Res. 115, 06205 (2010). doi:10.1029/2009JA014928

T.W. Hill, Interchange stability of a rapidly rotating magnetosphere. Planet. Space Sci. 24, 1151-1154 (1976)

T.W. Hill, Inertial limit on corotation. J. Geophys. Res. 25, 6554-6558 (1979)

T.W. Hill, Effect of the acceleration current on the centrifugal interchange instability. J. Geophys. Res. 111, A03214 (2006). doi:10.1029/2005JA011338

T.W. Hill, A.J. Dessler, L.J. Maher, Corotating magnetospheric convection. J. Geophys. Res. 86, 9020-9028 (1981). doi:10.1029/JA086iA11p09020

T.W. Hill, A.M. Rymer, J.L. Burch, F.J. Crary, D.T. Young, M.F. Thomsen, D. Delapp, N. André, A.J. Coates, G.R. Lewis, Evidence for rotationally driven plasma transport in Saturn's magnetosphere. Geophys. Res. Lett. 32, 14 (2005). doi:10.1029/2005GL022620 
T.W. Hill, M.F. Thomsen, M.G. Henderson, R.L. Tokar, A.J. Coates, H.J. McAndrews, G.R. Lewis, D.G. Mitchell, C.M. Jackman, C.T. Russell, M.K. Dougherty, F.J. Crary, D.T. Young, Plasmoids in Saturn's magnetotail. J. Geophys. Res. 113, 1214 (2008). doi:10.1029/2007JA012626

T.W. Hill, A.J. Dessler, C.K. Goertz, Magnetospheric models, ed. by A.J. Dessler 1983, pp. 353-394

T.S. Huang, T.W. Hill, Drift wave instability in the Io plasma torus. J. Geophys. Res. 96, 14075 (1991). doi:10.1029/91JA01170

D.E. Huddleston, R.J. Strangeway, J. Warnecke, C.T. Russell, M.G. Kivelson, F. Bagenal, Ion cyclotron waves in the Io torus during the Galileo encounter: Warm plasma dispersion analysis. Geophys. Res. Lett. 24, 2143 (1997). doi:10.1029/97GL01203

D.E. Huddleston, R.J. Strangeway, J. Warnecke, C.T. Russell, M.G. Kivelson, Ion cyclotron waves in the Io torus: Wave dispersion, free energy analysis, and $\mathrm{SO}_{2}^{+}$source rate estimates. J. Geophys. Res. 103, 19887-19900 (1998). doi:10.1029/97JE03557

D.E. Huddleston, R.J. Strangeway, X. Blanco-Cano, C.T. Russell, M.G. Kivelson, K.K. Khurana, Mirrormode structures at the Galileo-Io flyby: Instability criterion and dispersion analysis. J. Geophys. Res. 104, 17479-17490 (1999). doi:10.1029/1999JA900195

G. Ioaniddis, N. Brice, Plasma densities in the Jovian magnetosphere: plasma slingshot or Maxwell demon? Icarus 14, 360-373 (1971). doi:10.1016/0019-1035(71)90007-8

C.M. Jackman, C.S. Arridge, Solar cycle effects on the dynamics of Jupiter's and Saturn's magnetospheres. Solar Phys. 274(1-2), 481-502 (2011). doi:10.1007/s11207-011-9748-Z

C.M. Jackman, L. Lamy, M.P. Freeman, P. Zarka, B. Cecconi, W.S. Kurth, S.W.H. Cowley, M.K. Dougherty, On the character and distribution of lower-frequency radio emissions at Saturn and their relationship to substorm-like events. J. Geophys. Res. 114, 8211 (2009). doi:10.1029/2008JA013997

X. Jia, K.C. Hansen, T.I. Gombosi, M.G. Kivelson, G. Tóth, D.L. DeZeeuw, A.J. Ridley, Magnetospheric configuration and dynamics of Saturn's magnetosphere: A global MHD simulation. J. Geophys. Res. 117, 5225 (2012). doi: $10.1029 / 2012$ JA017575

R.E. Johnson, H.T. Smith, O.J. Tucker, M. Liu, M.H. Burger, E.C. Sittler, R.L. Tokar, The Enceladus and OH Tori at Saturn. Astrophys. J. Lett. 644, 137-139 (2006). doi:10.1086/505750

S.P. Joy, M.G. Kivelson, R.J. Walker, K.K. Khurana, C.T. Russell, T. Ogino, Probabilistic models of the Jovian magnetopause and bow shock locations. J. Geophys. Res. 107, 1309 (2002). doi:10.1029/ 2001JA009146

K. Kabin, M.R. Combi, T.I. Gombosi, D.L. DeZeeuw, K.C. Hansen, K.G. Powell, Io's magnetospheric interaction: an MHD model with day-night asymmetry. Planet. Space Sci. 49, 337-344 (2001)

S.J. Kanani, C.S. Arridge, G.H. Jones, A.N. Fazakerley, H.J. McAndrews, N. Sergis, S.M. Krimigis, M.K. Dougherty, A.J. Coates, D.T. Young, K.C. Hansen, N. Krupp, A new form of Saturn's magnetopause using a dynamic pressure balance model, based on in situ, multi-instrument Cassini measurements. J. Geophys. Res. 115, 6207 (2010). doi:10.1029/2009JA014262

M. Kane, D.G. Mitchell, J.F. Carbary, S.M. Krimigis, F.J. Crary, Plasma convection in Saturn's outer magnetosphere determined from ions detected by the Cassini INCA experiment. Geophys. Res. Lett. 35, 4102 (2008). doi:10.1029/2007GL032342

S. Kellett, C.S. Arridge, E.J. Bunce, A.J. Coates, S.W.H. Cowley, M.K. Dougherty, A.M. Persoon, N. Sergis, R.J. Wilson, Nature of the ring current in Saturn's dayside magnetosphere. J. Geophys. Res. 115, 8201 (2010). doi:10.1029/2009JA015146

S. Kellett, C.S. Arridge, E.J. Bunce, A.J. Coates, S.W.H. Cowley, M.K. Dougherty, A.M. Persoon, N. Sergis, R.J. Wilson, Saturn's ring current: Local time dependence and temporal variability. J. Geophys. Res. 116, 5220 (2011). doi:10.1029/2010JA016216

T.J. Kennelly, J.S. Leisner, G.B. Hospodarsky, D.A. Gurnett, Ordering of injection events within Saturnian SLS longitude and local time. J. Geophys. Res. 118, 832-838 (2013). doi:10.1002/jgra.50152

K. Khurana, M. Kivelson, M. Volwerk, The interactions of Europa and Callisto with the magnetosphere of Jupiter, in 34th COSPAR Scientific Assembly. COSPAR Meeting, vol. 34 (2002)

K.K. Khurana, M.K. Dougherty, C.T. Russell, J.S. Leisner, Mass loading of Saturn's magnetosphere near Enceladus. J. Geophys. Res. 112, 8203 (2007). doi:10.1029/2006JA012110

K.K. Khurana, X. Jia, M.G. Kivelson, F. Nimmo, G. Schubert, C.T. Russell, Evidence of a global magma ocean in Io's interior. Science 332, 1186 (2011). doi:10.1126/science.1201425

A. Kidder, R.M. Winglee, E.M. Harnett, Regulation of the centrifugal interchange cycle in Saturn's inner magnetosphere. J. Geophys. Res. 114, 2205 (2009). doi:10.1029/2008JA013100

A. Kidder, C.S. Paty, R.M. Winglee, E.M. Harnett, External triggering of plasmoid development at Saturn. J. Geophys. Res. 117, 7206 (2012). doi:10.1029/2012JA017625

M.G. Kivelson, D.J. Southwood, Dynamical consequences of two modes of centrifugal instability in Jupiter's outer magnetosphere. J. Geophys. Res. 110, 12209 (2005)

M.G. Kivelson, K.K. Khurana, R.J. Walker, J. Warnecke, C.T. Russell, J.A. Linker, D.J. Southwood, C. Polanskey, Io's interaction with the plasma torus: Galileo magnetometer report. Science 274, 396-398 (1996). doi:10.1126/science.274.5286.396 
M.G. Kivelson, K.K. Khurana, C.T. Russell, R.J. Walker, P.J. Coleman, F.V. Coroniti, J. Green, S. Joy, R.L. McPherron, C. Polanskey, D.J. Southwood, L. Bennett, J. Warnecke, D.E. Huddleston, Galileo at Jupiter-changing states of the magnetosphere and first looks at Io and Ganymede. Adv. Space Res. 20, 193-204 (1997a). doi:10.1016/S0273-1177(97)00533-4

M.G. Kivelson, K.K. Khurana, C.T. Russell, R.J. Walker, Intermittent short-duration magnetic field anomalies in the Io torus: Evidence for plasma interchange? Geophys. Res. Lett. 24, 2127 (1997b). doi:10.1029/ 97GL02202

A. Kopp, W.-H. Ip, Asymmetric mass loading effect at Titan's ionosphere. J. Geophys. Res. 106, 8323-8332 (2001). doi:10.1029/2000JA900140

H. Kriegel, S. Simon, U. Motschmann, J. Saur, F.M. Neubauer, A.M. Persoon, M.K. Dougherty, D.A. Gurnett, Influence of negatively charged plume grains on the structure of Enceladus' Alfvén wings: Hybrid simulations versus Cassini Magnetometer data. J. Geophys. Res. 116, 10223 (2011). doi:10.1029/ 2011JA016842

S.M. Krimigis, D.G. Mitchell, D.C. Hamilton, N. Krupp, S. Livi, E.C. Roelof, J. Dandouras, T.P. Armstrong, B.H. Mauk, C. Paranicas, P.C. Brandt, S. Bolton, A.F. Cheng, T. Choo, G. Gloeckler, J. Hayes, K.C. Hsieh, W.-H. Ip, S. Jaskulek, E.P. Keath, E. Kirsch, M. Kusterer, A. Lagg, L.J. Lanzerotti, D. LaVallee, J. Manweiler, R.W. McEntire, W. Rasmuss, J. Saur, F.S. Turner, D.J. Williams, J. Woch, Dynamics of Saturn's magnetosphere from MIMI during Cassini's orbital insertion. Science 307, 1270-1273 (2005). doi:10.1126/science. 1105978

W.S. Kurth, T.F. Averkamp, et al., An update to a Saturnian longitude system based on kilometric radio emissions. J. Geophys. Res. 113, 05222 (2008)

L. Lamy, Variability of southern and northern periodicities of Saturn Kilometric Radiation, in Planetary, Solar and Heliospheric Radio Emissions (PRE VII) (2011), pp. 38-50

L. Lamy, R. Prangé, W. Pryor, J. Gustin, S.V. Badman, H. Melin, T. Stallard, D.G. Mitchell, P.C. Brandt, Multispectral simultaneous diagnosis of Saturn's aurorae throughout a planetary rotation. J. Geophys. Res. 118 (2013). doi:10.1002/jgra.50404.

A.J. Lazarus, R.L. McNutt Jr., Low-energy plasma ion observations in Saturn's magnetosphere. J. Geophys. Res. 88, 8831-8846 (1983). doi:10.1029/JA088iA11p08831

S.A. Ledvina, S.H. Brecht, J.G. Luhmann, Ion distributions of 14 amu pickup ions associated with Titan's plasma interaction. Geophys. Res. Lett. 31, 17 (2004). doi:10.1029/2004GL019861

J.S. Leisner, C.T. Russell, K.K. Khurana, M.K. Dougherty, N. André, Warm flux tubes in the E-ring plasma torus: Initial Cassini magnetometer observations. Geophys. Res. Lett. 32, 14 (2005). doi:10.1029/ 2005GL022652

J.S. Leisner, C.T. Russell, M.K. Dougherty, X. Blanco-Cano, R.J. Strangeway, C. Bertucci, Ion cyclotron waves in Saturn's E ring: Initial Cassini observations. Geophys. Res. Lett. 33, 11101 (2006). doi:10.1029/2005GL024875

J.S. Leisner, C.T. Russell, H.Y. Wei, M.K. Dougherty, Probing Saturn's ion cyclotron waves on highinclination orbits: Lessons for wave generation. J. Geophys. Res. 116, 9235 (2011). doi:10.1029/ 2011JA016555

E. Lellouch, M.A. McGrath, K.L. Jessup, Io's atmosphere, ed. by R.M.C. Lopes, J.R. Spencer. Io After Galileo. A New View of Jupiter's Volcanic Moon. Springer Praxis Books/Geophysical Sciences (Springer, Berlin, 2007), pp. 231-264. doi:10.1007/978-3-540-48841-5_10

B. Levitt, D. Maslovsky, M.E. Mauel, Observation of centrifugally driven interchange instabilities in a plasma confined by a magnetic dipole. Phys. Rev. Lett. 94, 175002 (2005a). doi:10.1103/PhysRevLett. 94.175002

B. Levitt, D. Maslovsky, M.E. Mauel, J. Waksman, Excitation of the centrifugally driven interchange instability in a plasma confined by a magnetic dipolea). Phys. Plasmas 12(5), 055703 (2005b). doi:10.1063/ 1.1888685

J.A. Linker, K.K. Khurana, M.G. Kivelson, R.J. Walker, MHD simulations of Io's interaction with the plasma torus. J. Geophys. Res. 103, 19867 (1998)

A.S. Lipatov, M.R. Combi, Effects of kinetic processes in shaping Io's global plasma environment: A 3D hybrid model. Icarus 180, 412-427 (2006). doi:10.1016/j.icarus.2005.08.012

X. Liu, T.W. Hill, Effects of finite plasma pressure on centrifugally driven convection in Saturn's inner magnetosphere. J. Geophys. Res. 117, 7216 (2012). doi:10.1029/2012JA017827

X. Liu, T.W. Hill, R.A. Wolf, S. Sazykin, R.W. Spiro, H. Wu, Numerical simulation of plasma transport in Saturn's inner magnetosphere using the Rice Convection Model. J. Geophys. Res. 115, 12254 (2010). doi:10.1029/2010JA015859

P. Louarn, A. Roux, S. Perraut, W. Kurth, D. Gurnett, A study of the large-scale dynamics of the Jovian magnetosphere using the Galileo Plasma Wave Experiment. Geophys. Res. Lett. 25, 2905-2908 (1998). doi:10.1029/98GL01774 
P. Louarn, A. Roux, S. Perraut, W.S. Kurth, D.A. Gurnett, A study of the Jovian "energetic magnetospheric events" observed by Galileo: role in the radial plasma transport. J. Geophys. Res. 105, 13073-13088 (2000). doi:10.1029/1999JA900478

P. Louarn, B.H. Mauk, M.G. Kivelson, W.S. Kurth, A. Roux, C. Zimmer, D.A. Gurnett, D.J. Williams, A multi-instrument study of a Jovian magnetospheric disturbance. J. Geophys. Res. 106, 29883-29898 (2001). doi:10.1029/2001JA900067

Y.-J. Ma, A.F. Nagy, T.E. Cravens, I.V. Sokolov, J. Clark, K.C. Hansen, 3-D global MHD model prediction for the first close flyby of Titan by Cassini. Geophys. Res. Lett. 31, 22803 (2004). doi:10.1029/ 2004GL021215

H.R. Martens, D.B. Reisenfeld, J.D. Williams, R.E. Johnson, H.T. Smith, Observations of molecular oxygen ions in Saturn's inner magnetosphere. Geophys. Res. Lett. 35, 20103 (2008). doi:10.1029/ 2008GL035433

B.H. Mauk, S.A. Gary, M. Kane, E.P. Keath, S.M. Krimigis, T.P. Armstrong, Hot plasma parameters of Jupiter's inner magnetosphere. J. Geophys. Res. 101, 7685-7696 (1996). doi:10.1029/96JA00006

B.H. Mauk, D.J. Williams, R.W. McEntire, Energy-time dispersed charged particle signatures of dynamic injections in Jupiter's inner magnetosphere. Geophys. Res. Lett. 24, 2949-2952 (1997). doi:10.1029/ 97GL03026

B.H. Mauk, R.W. McEntire, D.J. Williams, A. Lagg, E.C. Roelof, S.M. Krimigis, T.P. Armstrong, T.A. Fritz, L.J. Lanzerotti, J.G. Roederer, B. Wilken, Galileo-measured depletion of near-Io hot ring current plasmas since the Voyager epoch. J. Geophys. Res. 103, 4715 (1998). doi:10.1029/97JA02343

B.H. Mauk, D.J. Williams, R.W. McEntire, K.K. Khurana, J.G. Roederer, Storm-like dynamics of Jupiter's inner and middle magnetosphere. J. Geophys. Res. 104, 22759-22778 (1999). doi:10.1029/ 1999JA900097

B.H. Mauk, J.T. Clarke, D. Grodent, J.H. Waite, C.P. Paranicas, D.J. Williams, Transient aurora on Jupiter from injections of magnetospheric electrons. Nature 415, 1003-1005 (2002)

B.H. Mauk, J. Saur, D.G. Mitchell, E.C. Roelof, P.C. Brandt, T.P. Armstrong, D.C. Hamilton, S.M. Krimigis, N. Krupp, S.A. Livi, J.W. Manweiler, C.P. Paranicas, Energetic particle injections in Saturn's magnetosphere. Geophys. Res. Lett. 32, 14 (2005). doi:10.1029/2005GL022485

B.H. Mauk, D.C. Hamilton, T.W. Hill, G.B. Hospodarsky, R.E. Johnson, C. Paranicas, E. Roussos, C.T. Russell, D.E. Shemansky, E.C. Sittler, R.M. Thorne, Fundamental plasma processes in Saturn's magnetosphere, in Saturn from Cassini-Huygens, ed. by M.K. Dougherty, L.W. Esposito, S.M. Krimigis (Springer, Berlin, 2009), p. 281. doi:10.1007/978-1-4020-9217-6_11

S. Maurice, M. Blanc, R. Prangé, E.C. Sittler, The magnetic-field-aligned polarization electric field and its effects on particle distribution in the magnetospheres of Jupiter and Saturn. Planet. Space Sci. 45, 14491465 (1997)

M.A. McGrath, E. Lellouch, D.F. Strobel, P.D. Feldman, R.E. Johnson, Satellite atmospheres, in Jupiter: The Planet, Satellites and Magnetosphere (2004), pp. 457-483

R.L. McNutt, J.W. Belcher, H.S. Bridge, Positive ion observations in the middle magnetosphere of Jupiter. J. Geophys. Res. 86, 8319-8342 (1981). doi:10.1029/JA086iA10p08319

R.L. Melrose, Rotational effects on the distribution of thermal plasma in the magnetosphere of Jupiter. Planet. Space Sci. 15, 381-393 (1967). doi:10.1016/0032-0633(67)90202-4

J.D. Menietti, J.B. Groene, T.F. Averkamp, G.B. Hospodarsky, W.S. Kurth, D.A. Gurnett, P. Zarka, Influence of Saturnian moons on Saturn kilometric radiation. J. Geophys. Res. 112, 8211 (2007). doi:10.1029/ 2007JA012331

D.G. Mitchell, S.M. Krimigis, C. Paranicas, P.C. Brandt, J.F. Carbary, E.C. Roelof, W.S. Kurth, D.A. Gurnett, J.T. Clarke, J.D. Nichols, J.-C. Gérard, D.C. Grodent, M.K. Dougherty, W.R. Pryor, Recurrent energization of plasma in the midnight-to-dawn quadrant of Saturn's magnetosphere, and its relationship to auroral UV and radio emissions. Planet. Space Sci. 57, 1732-1742 (2009). doi:10.1016/j.pss.2009.04.002

D.G. Mitchell, P.C. Brandt, J.F. Carbary, W.S. Kurth, S.M. Krimigis, C. Paranicas, N. Krupp, D.C. Hamilton, B.H. Mauk, G.B. Hospodarsky, M.K. Dougherty, W.R. Pryor, Injection, interchange and reconnection: Energetic particle observations in Saturn's magnetotail, in Magnetotails in the Solar System. AGU Geophysical Monograph Series (2015)

A.F. Nagy, Y. Liu, K.C. Hansen, K. Kabin, T.I. Gombosi, M.R. Combi, D.L. DeZeeuw, K.G. Powell, A.J. Kliore, The interaction between the magnetosphere of Saturn and Titan's ionosphere. J. Geophys. Res. 106, 6151-6160 (2001). doi:10.1029/2000JA000183

W.A. Newcomb, Convective instability induced by gravity in a plasma with a frozen-in magnetic field. Phys. Fluids 4, 391-396 (1961). doi:10.1063/1.1706342

J. Nichols, S. Cowley, Magnetosphere-ionosphere coupling currents in Jupiter's middle magnetosphere: effect of precipitation-induced enhancement of the ionospheric Pedersen conductivity. Ann. Geophys. 22, 1799-1827 (2004). doi:10.5194/angeo-22-1799-2004 
C. Paranicas, D.G. Mitchell, E.C. Roelof, P.C. Brandt, D.J. Williams, S.M. Krimigis, B.H. Mauk, Periodic intensity variations in global ENA images of Saturn. Geophys. Res. Lett. 32, 21101 (2005). doi:10.1029/ 2005GL023656

C. Paranicas, D.G. Mitchell, E.C. Roelof, B.H. Mauk, S.M. Krimigis, P.C. Brandt, M. Kusterer, F.S. Turner, J. Vandegriff, N. Krupp, Energetic electrons injected into Saturn's neutral gas cloud. Geophys. Res. Lett. 34, 2109 (2007). doi:10.1029/2006GL028676

D.H. Pontius Jr., T.W. Hill, Rotation driven plasma transport-the coupling of macroscopic motion and microdiffusion. J. Geophys. Res. 94, 15041-15053 (1989). doi:10.1029/JA094iA11p15041

D.H. Pontius Jr., T.W. Hill, M.E. Rassbach, Steady state plasma transport in a corotation-dominated magnetosphere. Geophys. Res. Lett. 13, 1097-1100 (1986). doi:10.1029/GL013i011p01097

D.H. Pontius, T.W. Hill, Plasma mass loading from the extended neutral gas torus of Enceladus as inferred from the observed plasma corotation lag. Geophys. Res. Lett. 36, 23103 (2009). doi:10.1029/ 2009GL041030

D.H. Pontius, R.A. Wolf, T.W. Hill, R.W. Spiro, Y.S. Yang, W.H. Smyth, Velocity shear impoundment of the Io plasma torus. J. Geophys. Res. 103, 19935-19946 (1998). doi:10.1029/98JE00538

W.R. Pryor, A.M. Rymer, D.G. Mitchell, T.W. Hill, D.T. Young, J. Saur, G.H. Jones, S. Jacobsen, S.W.H. Cowley, B.H. Mauk, A.J. Coates, J. Gustin, D. Grodent, J.-C. Gérard, L. Lamy, J.D. Nichols, S.M. Krimigis, L.W. Esposito, M.K. Dougherty, A.J. Jouchoux, A.I.F. Stewart, W.E. McClintock, G.M. Holsclaw, J.M. Ajello, J.E. Colwell, A.R. Hendrix, F.J. Crary, J.T. Clarke, X. Zhou, The auroral footprint of Enceladus on Saturn. Nature 472, 331-333 (2011). doi:10.1038/nature09928

M. Rodriguez-Martinez, X. Blanco-Cano, C. Russell, J.S. Leisner, M.M. Cowee, M.K. Dougherty, Harmonic growth of ion cyclotron waves in Saturn's Magnetosphere, in 37th COSPAR Scientific Assembly. COSPAR Meeting, vol. 37 (2008), p. 2638

M. Rodríguez-Martínez, X. Blanco-Cano, C.T. Russell, J.S. Leisner, R.J. Wilson, M.K. Dougherty, Harmonic growth of ion-cyclotron waves in Saturn's magnetosphere. J. Geophys. Res. 115, 9207 (2010). doi:10.1029/2009JA015000

A. Runov, V. Angelopoulos, X.-Z. Zhou, X.-J. Zhang, S. Li, F. Plaschke, J. Bonnell, A THEMIS multicase study of dipolarization fronts in the magnetotail plasma sheet. J. Geophys. Res. 116, 5216 (2011). doi:10.1029/2010JA016316

C.T. Russell, D.E. Huddleston, Ion-cyclotron waves at Io. Adv. Space Res. 26, 1505-1511 (2000)

C.T. Russell, M.G. Kivelson, Detection of SO in Io's exosphere. Science 287, 1998-1999 (2000). doi:10.1126/science.287.5460.1998

C.T. Russell, D.E. Huddleston, R.J. Strangeway, X. Blanco-Cano, M.G. Kivelson, K.K. Khurana, L.A. Frank, W. Paterson, D.A. Gurnett, W.S. Kurth, Mirror-mode structures at the Galileo-Io flyby: Observations. J. Geophys. Res. 104, 17471-17478 (1999). doi:10.1029/1999JA900202

C.T. Russell, M.G. Kivelson, K.K. Khurana, D.E. Huddleston, Circulation and dynamics in the Jovian magnetosphere. Adv. Space Res. 26, 1671-1676 (2000). doi:10.1016/S0273-1177(00)00115-0

C.T. Russell, Y.L. Wang, X. Blanco-Cano, R.J. Strangeway, The Io mass-loading disk: Constraints provided by ion cyclotron wave observations. J. Geophys. Res. 106, 26233-26242 (2001). doi:10.1029/ 2001JA900029

C.T. Russell, X. Blanco-Cano, M.G. Kivelson, Ion cyclotron waves in Io's wake region. Planet. Space Sci. 51, 233-238 (2003a). doi:10.1016/S0032-0633(02)00198-8

C.T. Russell, X. Blanco-Cano, Y.L. Wang, M.G. Kivelson, Ion cyclotron waves at Io: implications for the temporal variation of Io's atmosphere. Planet. Space Sci. 51, 937-944 (2003b). doi:10.1016/ j.pss.2003.05.005

C.T. Russell, M.G. Kivelson, K.K. Khurana, Statistics of depleted flux tubes in the Jovian magnetosphere. Planet. Space Sci. 53, 937-943 (2005). doi:10.1016/j.pss.2005.04.007

C.T. Russell, J.S. Leisner, C.S. Arridge, M.K. Dougherty, X. Blanco-Cano, Nature of magnetic fluctuations in Saturn's middle magnetosphere. J. Geophys. Res. 111, 12205 (2006). doi:10.1029/2006JA011921

A.M. Rymer, B.H. Mauk, T.W. Hill, C. Paranicas, N. André, E.C. Sittler, D.G. Mitchell, H.T. Smith, R.E. Johnson, A.J. Coates, D.T. Young, S.J. Bolton, M.F. Thomsen, M.K. Dougherty, Electron sources in Saturn's magnetosphere. J. Geophys. Res. 112, 2201 (2007). doi:10.1029/2006JA012017

A.M. Rymer, B.H. Mauk, T.W. Hill, C. Paranicas, D.G. Mitchell, A.J. Coates, D.T. Young, Electron circulation in Saturn's magnetosphere. J. Geophys. Res. 113, 1201 (2008). doi:10.1029/2007JA012589

A.M. Rymer, B.H. Mauk, T.W. Hill, N. André, D.G. Mitchell, C. Paranicas, D.T. Young, H.T. Smith, A.M. Persoon, J.D. Menietti, G.B. Hospodarsky, A.J. Coates, M.K. Dougherty, Cassini evidence for rapid interchange transport at Saturn. Planet. Space Sci. 57, 1779-1784 (2009). doi:10.1016/j.pss.2009.04.010

J. Saur, D.F. Strobel, F.M. Neubauer, Interaction of the Jovian magnetosphere with Europa: Constraints on the neutral atmosphere. J. Geophys. Res. 103, 19947-19962 (1998). doi:10.1029/97JE03556

J. Saur, F.M. Neubauer, D.F. Strobel, M.E. Summers, Three-dimensional plasma simulation of Io's interaction with the Io plasma torus: Asymmetric plasma flow. J. Geophys. Res. 104, 25105-25126 (1999). doi:10.1029/1999JA900304 
J. Saur, F.M. Neubauer, D.F. Strobel, M.E. Summers, Interpretation of Galileo's Io plasma and field observations: I0, I24, and I27 flybys and close polar passes. J. Geophys. Res. 107, 1422 (2002). doi:10.1029/ 2001JA005067

J. Saur, F.M. Neubauer, J.E.P. Connerney, P. Zarka, M.G. Kivelson, Plasma interaction of Io with its plasma torus, in Jupiter: The Planet, Satellites and Magnetosphere (2004), pp. 537-560

P. Schippers, M. Blanc, N. André, I. Dandouras, G.R. Lewis, L.K. Gilbert, A.M. Persoon, N. Krupp, D.A. Gurnett, A.J. Coates, S.M. Krimigis, D.T. Young, M.K. Dougherty, Multi-instrument analysis of electron populations in Saturn's magnetosphere. J. Geophys. Res. 113, 7208 (2008). doi:10.1029/2008JA013098

M. Schulz, Jupiter's radiation belts. Space Sci. Rev. 23, 277-318 (1979). doi:10.1007/BF00173813

N. Sergis, S.M. Krimigis, D.G. Mitchell, D.C. Hamilton, N. Krupp, B.M. Mauk, E.C. Roelof, M. Dougherty, Ring current at Saturn: Energetic particle pressure in Saturn's equatorial magnetosphere measured with Cassini/MIMI. Geophys. Res. Lett. 34, 09102 (2007). doi:10.1029/2006GL029223

D.E. Shemansky, Energy branching in the Io plasma torus: The failure of neutral cloud theory. J. Geophys. Res. 93, 1773 (1988)

S. Simon, G. Kleindienst, A. Boesswetter, T. Bagdonat, U. Motschmann, K.-H. Glassmeier, J. Schuele, C. Bertucci, M.K. Dougherty, Hybrid simulation of Titan's magnetic field signature during the Cassini T9 flyby. Geophys. Res. Lett. 34, 24 (2007). doi:10.1029/2007GL029967

G.L. Siscoe, A. Eviatar, R.M. Thorne, J.D. Richardson, F. Bagenal, J.D. Sullivan, Ring current impoundment of the Io plasma torus. J. Geophys. Res. 86, 8480-8484 (1981). doi:10.1029/JA086iA10p08480

E.C. Sittler, M. Thomsen, R.E. Johnson, R.E. Hartle, M. Burger, D. Chornay, M.D. Shappirio, D. Simpson, H.T. Smith, A.J. Coates, A.M. Rymer, D.J. McComas, D.T. Young, D. Reisenfeld, M. Dougherty, N. Andre, Erratum to "Cassini observations of Saturn's inner plasmasphere: Saturn orbit insertion results”. [Planetary and Space Science 54 (2006) 1197-1210]. Planet. Space Sci. 55, 2218-2220 (2007). doi:10.1016/j.pss.2006.11.022

E.C. Sittler, N. Andre, M. Blanc, M. Burger, R.E. Johnson, A. Coates, A. Rymer, D. Reisenfeld, M.F. Thomsen, A. Persoon, M. Dougherty, H.T. Smith, R.A. Baragiola, R.E. Hartle, D. Chornay, M.D. Shappirio, D. Simpson, D.J. McComas, D.T. Young, Ion and neutral sources and sinks within Saturn's inner magnetosphere: Cassini results. Planet. Space Sci. 56, 3-18 (2008). doi:10.1016/j.pss.2007.06.006

T.E. Skinner, S.T. Durrance, Neutral oxygen and sulfur densities in the Io torus. Astrophys. J. 310, 966-971 (1986). doi:10.1086/164747

C.G.A. Smith, A.D. Aylward, Coupled rotational dynamics of Jupiter's thermosphere and magnetosphere. Ann. Geophys. 27, 199-230 (2009)

E.J. Smith, B.T. Tsurutani, Saturn's magnetosphere-observations of ion cyclotron waves near the Dione L shell. J. Geophys. Res. 88, 7831-7836 (1983). doi:10.1029/JA088iA10p07831

W.H. Smyth, M.L. Marconi, Nature of the Iogenic plasma source in Jupiter's magnetosphere I. Circumplanetary distribution. Icarus 166(1), 85-106 (2003)

D. Snowden, R. Winglee, C. Bertucci, M. Dougherty, Three-dimensional multifluid simulation of the plasma interaction at Titan. J. Geophys. Res. 112, 12221 (2007). doi:10.1029/2007JA012393

D. Snowden, R. Winglee, A. Kidder, Titan at the edge: 1. Titan's interaction with Saturn's magnetosphere in the prenoon sector. J. Geophys. Res. 116, 8229 (2011a). doi:10.1029/2011JA016435

D. Snowden, R. Winglee, A. Kidder, Titan at the edge: 2. A global simulation of Titan exiting and reentering Saturn's magnetosphere at 13:16 Saturn local time. J. Geophys. Res. 116, 8230 (2011b). doi:10.1029/ 2011JA016436

D.J. Southwood, M.G. Kivelson, Magnetospheric interchange instability. J. Geophys. Res. 92, 109-116 (1987). doi:10.1029/JA092iA01p00109

D.J. Southwood, M.G. Kivelson, Magnetospheric interchange motions. J. Geophys. Res. 94, 299-308 (1989)

D.J. Southwood, M.G. Kivelson, A new perspective concerning the influence of the solar wind on the Jovian magnetosphere. J. Geophys. Res. 106, 6123-6130 (2001). doi:10.1029/2000JA000236

K. Szego, Z. Bebesi, G. Erdos, L. Foldy, F. Crary, D.J. McComas, D.T. Young, S. Bolton, A.J. Coates, A.M. Rymer, R.E. Hartle, E.C. Sittler, D. Reisenfeld, J.J. Bethelier, R.E. Johnson, H.T. Smith, T.W. Hill, J. Vilppola, J. Steinberg, N. Andre, The global plasma environment of Titan as observed by Cassini Plasma Spectrometer during the first two close encounters with Titan. Geophys. Res. Lett. 32, 20 (2005). doi:10.1029/2005GL022646

N. Thomas, F. Bagenal, T.W. Hill, J.K. Wilson, The Io neutral clouds and plasma torus, in Jupiter. The Planet, Satellites and Magnetosphere, ed. by F. Bagenal, T.E. Dowling, W.B. McKinnon (2004), pp. 561-591

M.F. Thomsen, D.B. Reisenfeld, D.M. Delapp, R.L. Tokar, D.T. Young, F.J. Crary, E.C. Sittler, M.A. McGraw, J.D. Williams, Survey of ion plasma parameters in Saturn's magnetosphere. J. Geophys. Res. 115, 10220 (2010). doi:10.1029/2010JA015267

M.F. Thomsen, E. Roussos, M. Andriopoulou, P. Kollmann, C.S. Arridge, C.P. Paranicas, D.A. Gurnett, R.L. Powell, R.L. Tokar, D.T. Young, Saturn's inner magnetospheric convection pattern: Further evidence. J. Geophys. Res. 117, 9208 (2012). doi:10.1029/2011JA017482 
R.M. Thorne, Radiation belt dynamics: The importance of wave-particle interactions. Geophys. Res. Lett. 37, 22107 (2010). doi:10.1029/2010GL044990

R.M. Thorne, T.P. Armstrong, S. Stone, D.J. Williams, R.W. McEntire, S.J. Bolton, D.A. Gurnett, M.G. Kivelson, Galileo evidence for rapid interchange transport in the Io torus. Geophys. Res. Lett. 24, 2131 (1997). doi:10.1029/97GL01788

R.L. Tokar, R.E. Johnson, T.W. Hill, D.H. Pontius, W.S. Kurth, F.J. Crary, D.T. Young, M.F. Thomsen, D.B. Reisenfeld, A.J. Coates, G.R. Lewis, E.C. Sittler, D.A. Gurnett, The interaction of the atmosphere of Enceladus with Saturn's plasma. Science 311, 1409-1412 (2006). doi:10.1126/science.1121061

R.L. Tokar, R.J. Wilson, R.E. Johnson, M.G. Henderson, M.F. Thomsen, M.M. Cowee, E.C. Sittler, D.T. Young, F.J. Crary, H.J. McAndrews, H.T. Smith, Cassini detection of water-group pick-up ions in the Enceladus torus. Geophys. Res. Lett. 35, 14202 (2008). doi:10.1029/2008GL034749

V.M. Vasyliūnas, Mathematical models of magnetospheric convection and its coupling to the ionosphere, in Particles and Field in the Magnetosphere, ed. by B.M. McCormack, A. Renzini. Astrophysics and Space Science Library, vol. 17 (1970), p. 60

V.M. Vasyliūnas, Plasma distribution and flow, in Physics of the Jovian Magnetosphere, ed. by A.J. Dessler (Cambridge University Press, New York, 1983), pp. 395-453. ISBN 0521520061 (paperback)

V.M. Vasyliūnas, Physical origin of pickup currents, in European Planetary Science Congress (2006)

V.M. Vasyliūnas, Comparing Jupiter and Saturn: dimensionless input rates from plasma sources within the magnetosphere. Ann. Geophys. 26, 1341-1343 (2008)

V.M. Vasyliūnas, D.H. Pontius, Rotationally driven interchange instability: Reply to André and Ferrière. J. Geophys. Res. 112(A10), A10204 (2007). doi:10.1029/2007JA012457

M. Volwerk, K.K. Khurana, Ion pick-up near the icy Galilean satellites, in American Institute of Physics Conference Series, ed. by J. Le Roux, G.P. Zank, A.J. Coates, V. Florinski. American Institute of Physics Conference Series, vol. 1302 (2010), pp. 263-269. doi:10.1063/1.3529982

M. Volwerk, M.G. Kivelson, K.K. Khurana, Wave activity in Europa's wake: Implications for ion pickup. J. Geophys. Res. 106, 26033-26048 (2001). doi:10.1029/2000JA000347

J.H. Waite, M.R. Combi, W.-H. Ip, T.E. Cravens, R.L. McNutt, W. Kasprzak, R. Yelle, J. Luhmann, H. Niemann, D. Gell, B. Magee, G. Fletcher, J. Lunine, W.-L. Tseng, Cassini ion and neutral mass spectrometer: Enceladus plume composition and structure. Science 311, 1419-1422 (2006). doi:10.1126/ science. 1121290

M. Walt, Introduction to Geomagnetically Trapped Radiation. Cambridge Atmospheric and Space Science Series, vol. 10 (1994)

Y. Wang, C.T. Russell, J. Raeder, The Io mass-loading disk: Model calculations. J. Geophys. Res. 106, 2624326260 (2001). doi:10.1029/2001JA900062

J. Warnecke, M.G. Kivelson, K.K. Khurana, D.E. Huddleston, C.T. Russell, Ion cyclotron waves observed at Galileo's Io encounter: Implications for neutral cloud distribution and plasma composition. Geophys. Res. Lett. 24, 2139 (1997). doi:10.1029/97GL01129

D.J. Williams, B. Mauk, R.E. McEntire, E.C. Roelof, S.M. Krimigis, T.P. Armstrong, B. Wilken, J.G. Roederer, T.A. Fritz, L.J. Lanzerotti, Energetic electron beams measured at Io. Bull. Am. Astron. Soc. 28, 1055 (1996)

R.J. Wilson, R.L. Tokar, M.G. Henderson, T.W. Hill, M.F. Thomsen, D.H. Pontius, Cassini plasma spectrometer thermal ion measurements in Saturn's inner magnetosphere. J. Geophys. Res. 113, 12218 (2008). doi:10.1029/2008JA013486

R.M. Winglee, D. Snowden, A. Kidder, Modification of Titan's ion tail and the Kronian magnetosphere: Coupled magnetospheric simulations. J. Geophys. Res. 114, 5215 (2009). doi:10.1029/2008JA013343

R.M. Winglee, A. Kidder, E. Harnett, N. Ifland, C. Paty, D. Snowden, Generation of periodic signatures at Saturn through Titan's interaction with the centrifugal interchange instability. J. Geophys. Res. 118, 4253-4269 (2013). doi:10.1002/jgra.50397

R.A. Wolf, Computer model of inner magnetospheric convection, in Solar-Terrestrial Physics: Principles and Theoretical Foundations, ed. by R.L. Carovillano, J.M. Forbes. Astrophysics and Space Science Library, vol. 104 (1983), p. 342

M.C. Wong, W.H. Smyth, Model calculations for Io's atmosphere at Eastern and Western elongations. Icarus 146, 60-74 (2000). doi:10.1006/icar.2000.6362

H. Wu, T.W. Hill, R.A. Wolf, R.W. Spiro, Numerical simulation of fine structure in the Io plasma torus produced by the centrifugal interchange instability. J. Geophys. Res. 112, 2206 (2007). doi:10.1029/ 2006JA012032

Y.S. Yang, R.A. Wolf, R.W. Spiro, A.J. Dessler, Numerical simulation of plasma transport driven by the Io torus. Geophys. Res. Lett. 19, 957-960 (1992). doi:10.1029/92GL01031

Y.S. Yang, R.A. Wolf, R.W. Spiro, T.W. Hill, A.J. Dessler, Numerical simulation of torus-driven plasma transport in the Jovian magnetosphere. J. Geophys. Res. 99, 8755-8770 (1994). doi:10.1029/94JA00142 
J.N. Yates, N. Achilleos, P. Guio, Influence of upstream solar wind on thermospheric flows at Jupiter. Planet. Space Sci. 61, 15-31 (2012). doi:10.1016/j.pss.2011.08.007

D.T. Young, J.J. Berthelier, M. Blanc, J.L. Burch, A.J. Coates, R. Goldstein, M. Grande, T.W. Hill, R.E. Johnson, V. Kelha, D.J. McComas, E.C. Sittler, K.R. Svenes, K. Szegö, P. Tanskanen, K. Ahola, D. Anderson, S. Bakshi, R.A. Baragiola, B.L. Barraclough, R.K. Black, S. Bolton, T. Booker, R. Bowman, P. Casey, F.J. Crary, D. Delapp, G. Dirks, N. Eaker, H. Funsten, J.D. Furman, J.T. Gosling, H. Hannula, C. Holmlund, H. Huomo, J.M. Illiano, P. Jensen, M.A. Johnson, D.R. Linder, T. Luntama, S. Maurice, K.P. McCabe, K. Mursula, B.T. Narheim, J.E. Nordholt, A. Preece, J. Rudzki, A. Ruitberg, K. Smith, S. Szalai, M.F. Thomsen, K. Viherkanto, J. Vilppola, T. Vollmer, T.E. Wahl, M. Wüest, T. Ylikorpi, C. Zinsmeyer, Cassini plasma spectrometer investigation. Space Sci. Rev. 114, 1-4 (2004). doi:10.1007/ s11214-004-1406-4

B. Zieger, K.C. Hansen, T.I. Gombosi, D.L. De Zeeuw, Periodic plasma escape from the mass-loaded Kronian magnetosphere. J. Geophys. Res. 115, 8208 (2010). doi:10.1029/2009JA014951 